\title{
La variante infinitésimale de la formule des traces de Jacquet-Rallis pour les groupes linéaires
}

\author{
Michał Zydor *
}

\begin{abstract}
We establish an infinitesimal version of the Jacquet-Rallis trace formula for general linear groups. Our formula is obtained by integrating a kernel truncated à la Arthur multiplied by the absolute value of the determinant to the power $s \in \mathbb{C}$. It has a geometric side which is a sum of distributions $I_{\mathfrak{o}}(s, \cdot)$ indexed by the invariants of the adjoint action of $\mathrm{GL}_{n}(\mathrm{~F})$ on $\mathfrak{g l}_{n+1}(\mathrm{~F})$ as well as a "spectral side" consisting of the Fourier transforms of the aforementioned distributions. We prove that the distributions $I_{\mathfrak{o}}(s, \cdot)$ are invariant and depend only on the choice of the Haar measure on $\mathrm{GL}_{n}(\mathbb{A})$. For regular semi-simple classes $\mathfrak{o}, I_{\mathfrak{o}}(s, \cdot)$ is a relative orbital integral of Jacquet-Rallis. For classes $\mathfrak{o}$ called relatively regular semi-simple, we express $I_{\mathfrak{o}}(s, \cdot)$ in terms of relative orbital integrals regularised by means of zeta functions.
\end{abstract}

\section{Résumé}

Nous établissons une variante infinitésimale de la formule des traces de Jacquet-Rallis pour les groupes linéaires. Notre formule s'obtient par intégration d'un noyau tronqué à la Arthur multiplié par la valeur absolue du déterminant à la puissance $s \in \mathbb{C}$. Elle possède un côté géométrique qui est une somme de distributions $I_{\mathfrak{o}}(s, \cdot)$ indexée par les invariants de l'action de $\mathrm{GL}_{n}(\mathrm{~F})$ sur $\mathfrak{g l}_{n+1}(\mathrm{~F})$ ainsi qu'un "côté spectral" formé des transformées de Fourier des distributions précédentes. On démontre que les distributions $I_{\mathfrak{o}}(s, \cdot)$ sont invariantes et ne dépendent que du choix de la mesure de Haar sur $\mathrm{GL}_{n}(\mathbb{A})$. Pour des classes o semi-simples régulières $I_{\mathfrak{o}}(s, \cdot)$ est une intégrale orbitale relative de Jacquet-Rallis. Pour les classes $\mathfrak{o}$ dites relativement semi-simples régulières, on exprime $I_{\mathfrak{o}}(s, \cdot)$ en terme des intégrales orbitales relatives régularisées à l'aide des fonctions zêta.

\section{Table des matières}

0 Introduction 2

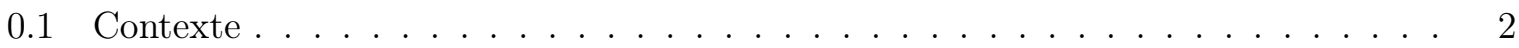

0.2 Nos résultats $\ldots \ldots \ldots \ldots \ldots$

1 Prolégomènes 5

1.1 Préliminaires pour la formule des traces . . . . . . . . . . . . . 5

1.2 Le domaine de Siegel . . . . . . . . . . . . . . . . . . . 7 7

1.3 Algèbres de Lie . . . . . . . . . . . . . . . . . . . . . . . . 7

1.4 Fonctions de Bruhat-Schwartz . . . . . . . . . . . . . . . 8

1.5 Les mesures de Haar . . . . . . . . . . . . . . . . . . 8

$1.6 \mathrm{GL}_{n} \hookrightarrow \mathrm{GL}_{n+1} \ldots \ldots \ldots \ldots \ldots \ldots \ldots \ldots$

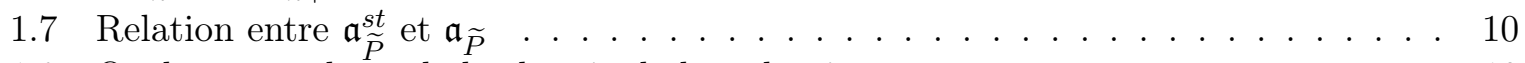

1.8 Quelques résultats de la théorie de la réduction . . . . . . . . . . . . . 10

1.9 Majorations cruciales . . . . . . . . . . . . . . . . . . . 11

*Université Paris Diderot Institut de Mathématiques de Jussieu-Paris Rive Gauche UMR7586 Bâtiment Sophie Germain Case 701275205 PARIS Cedex 13 France mail : michal.zydor@imj-prg.fr 
2 Formule des traces relative pour $\mathfrak{g l}(n+1) / / \mathrm{GL}(n) \quad \mathbf{1 2}$

2.1 Les invariants . . . . . . . . . . . . . . . . . . . . . . 12

2.2 Convergence du noyau modifié . . . . . . . . . . . . . . . . . . . . 13

3 Propriétés qualitatives $\quad \mathbf{1 6}$

3.1 Polynômes-exponentielles . . . . . . . . . . . . . . . . . . . . . 17

3.2 Une généralisation du théorème $2.6 \ldots \ldots \ldots$

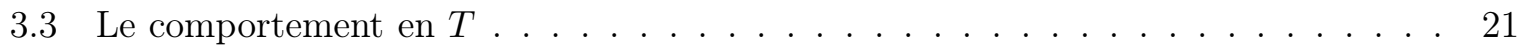

3.4 Équivariance . . . . . . . . . . . . . . . . . . . . . . . . 23

3.5 Indépendance des choix . . . . . . . . . . . . . . . . . . . . 24

3.6 Orbites semi-simples régulières . . . . . . . . . . . . . . . 25

4 Formule des traces infinitésimale

5 Orbites semi-simples 26

5.1 Notations . . . . . . . . . . . . . . . . . . . . . . 26

5.2 Orbites dans une classe relativement semi-simple régulière . . . . . . . . . . 27

5.3 Quelques définitions associées aux orbites . . . . . . . . . . . . . . 29

5.4 Le résultat principal . . . . . . . . . . . . . . . . . . . . 29

5.5 Deuxième formule pour le noyau tronqué . . . . . . . . . . . . . . . . 33

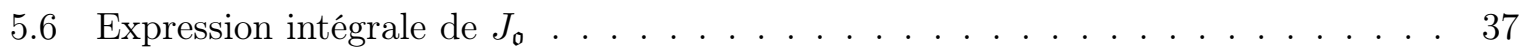

5.7 Résultats d'holomorphie . . . . . . . . . . . . . . . . . . . . 38

\section{Introduction}

\subsection{Contexte}

Dans [JR11, Jacquet et Rallis proposent une approche via une formule des traces relative à la conjecture globale de Gan-Gross-Prasad (GGP) pour les groupes unitaires GGP12. Ils proposent une existence de deux formules des traces relatives, une pour les groupes unitaires et une pour les groupes linéaires. Les deux formules ont des côtés géométriques et spectraux. Le comparaison de côtés géométriques de ces deux formules doit mener aux résultats spectraux qui entament la conjecture de Gan-Gross-Prasad. Une version simple de cette formule, c'est-à-dire une version valable pour une certaine classe de fonctions test, a été utilisé par Zhang pour démontrer une partie substantielle de la conjecture GGP [Zha14b, Zha14a] ainsi que certains cas du raffinement de la conjecture GGP du à Ichino et Ikeda [II10] et N. Harris [Har14].

\subsection{Nos résultats}

Afin qu'on puisse étendre les résultat de Zhang il faut des formules des traces valables pour toutes les fonctions lisses à support compact. Cet article étudie la variante infinitésimale du côté géométrique de la formule des traces relative de Jacquet-Rallis pour les groupes linéaires et il est précédé par Zyd15a où on étudie la variante infinitésimale du côté géométrique pour les groupes unitaires. Les côtés géométriques pour les groupes ainsi que les côtés spectraux seront présentés dans [Zyd15b].

Soient $\mathrm{E} / \mathrm{F}$ une extension quadratique de corps de nombres, $\mathbb{A}$ l'anneau des adèles de $\mathrm{F}$ et $\eta: \mathrm{F}^{*} \backslash \mathbb{A}^{*} \rightarrow \mathbb{C}$ le caractère associé à l'extension $\mathrm{E} / \mathrm{F}$ par la théorie de corps de classes. Soit $n \in \mathbb{N}$ et notons $G=\mathrm{GL}_{n}$ que l'on voit comme un sous-groupe de $\widetilde{G}=\mathrm{GL}_{n+1}$ par le plongement diagonal $g \mapsto\left(\begin{array}{cc}g & \\ & 1\end{array}\right)$. Soit $S_{n+1}$ la sous-F-variété de $\operatorname{Res}_{\mathrm{E} / \mathrm{F}} \widetilde{G}$ composée de $g \in \operatorname{Res}_{\mathrm{E} / \mathrm{F}} \widetilde{G}$ tels que $g \bar{g}=1$ où $\bar{g}$ c'est le conjugué de $g$ par l'élément non-trivial du groupe de Galois de E/F. On 
voit $G$ et $\widetilde{G}$ comme des sous-F-groupes de $\operatorname{Res}_{\mathrm{E} / \mathrm{F}} \widetilde{G}$. L'action de $G$ sur $\operatorname{Res}_{\mathrm{E} / \mathrm{F}} \widetilde{G}$ stabilise alors $S_{n+1}$.

Du côté géométrique pour le groupe linéaire de la formule des traces relative de Jacquet-Rallis on étudie l'intégrale formelle

$$
\int_{G(\mathrm{~F}) \backslash G(\mathbb{A})} k_{f}(x) \eta(\operatorname{det} x) d x
$$

où $f \in C_{c}^{\infty}\left(S_{n+1}(\mathbb{A})\right)$ est une fonction lisse à support compact sur $S_{n+1}(\mathbb{A})$ et $k_{f}(x)$ égale $\sum_{\gamma \in S_{n+1}(\mathrm{~F}} f\left(x^{-1} \gamma x\right)$ pour $x \in G(\mathrm{~F}) \backslash G(\mathbb{A})$. L'intégrale n'est pas bien définie pour toutes les fonctions $f$. Zhang utilise cet intégrale pour des fonctions vérifiant certaines conditions du support locale pour lesquelles elle converge et admet une décomposition en une somme des intégrales orbitales relatives.

Dans cet article on s'intéresse à la version infinitésimale de l'intégrale (0.1). Soient $\widetilde{\mathfrak{g}}=\operatorname{Lie}(\widetilde{G})$ et $\mathfrak{s}_{n+1}$ l'espace tangent à l'identité de $S_{n+1}$. Alors, $\mathfrak{s}_{n+1}$, vu comme un sous-espace de $\operatorname{Res}_{\mathrm{E} / \mathrm{F}} \widetilde{\mathfrak{g}}$, c'est :

$$
\mathfrak{s}_{n+1}=\left\{X \in \operatorname{Res}_{\mathrm{E} / \mathrm{F}} \widetilde{\mathfrak{g}}_{n+1} \mid X+\bar{X}=0\right\} .
$$

Si on choisit un $\tau \in \mathrm{E}$ tel que $\bar{\tau}=-\tau$, la multiplication par $\tau$ induit un isomorphisme entre $\mathfrak{s}_{n+1}$ et $\widetilde{\mathfrak{g}}$. Le groupe $G$ agit sur $\mathfrak{s}_{n+1}$ et sur $\widetilde{\mathfrak{g}}$ par adjonction. Cette identification est alors $G$-équivariante. Soit $s \in \mathbb{C}$. L'analogue infinitésimal de l'intégrale (0.1) que l'on considère c'est

$$
I(s, f)=\int_{G(\mathrm{~F}) \backslash G(\mathbb{A})} k_{f}(x)|\operatorname{det} x|_{\mathbb{A}}^{s} \eta(\operatorname{det} x) d x, \quad k_{f}(x)=\sum_{\xi \in \widetilde{\mathfrak{g}}(\mathrm{F})} f\left(x^{-1} \xi x\right), f \in \mathcal{S}(\widetilde{\mathfrak{g}}(\mathbb{A})),
$$

où $|\cdot|_{\mathbb{A}}$ c'est la valeur absolue standard sur le groupe des idèles de $\mathbb{A}$ et $\mathcal{S}(\widetilde{\mathfrak{g}}(\mathbb{A}))$ c'est l'espace des fonctions de type Bruhat-Schwartz sur $\widetilde{\mathfrak{g}}(\mathbb{A})$. Dans cet article on définit une régularisation de cette intégrale par un processus de troncature à la Arthur et on étudie les propriétés de la distribution ainsi obtenue. Décrivons brièvement cette troncature maintenant.

Fixons $B$ un sous-groupe de Borel de $G$ ainsi que sa décomposition de Levi $B=M_{0} N_{0}$ avec $M_{0}$ une partie de Levi de $B$ et $N_{0}$ son radical unipotent. On note $\mathcal{F}(B)$ l'ensemble des sousgroupes paraboliques de $G$ contenant $B$. Tout $P \in \mathcal{F}(B)$ admet alors une unique décomposition de Levi $P=M_{P} N_{P}$. Soit $M_{\widetilde{0}}$ l'unique sous-groupe de Levi minimal de $\widetilde{G}$ contenant $M_{0}$. On note $\mathcal{F}\left(M_{\widetilde{0}}, B\right)$ l'ensemble des sous-groupes paraboliques de $\widetilde{G}$ contenant $B$. Tout $\widetilde{P} \in \mathcal{F}\left(M_{\widetilde{0}}, B\right)$ admet alors une unique décomposition de Levi $\widetilde{P}=M_{\widetilde{P}} N_{\widetilde{P}}$ où $M_{0} \subseteq M_{\widetilde{P}}$. Pour tout $\widetilde{P} \in$ $\mathcal{F}\left(M_{\widetilde{0}}, B\right)$ on a $\widetilde{P} \cap G \in \mathcal{F}(B)$. On note alors $P:=\widetilde{P} \cap G$.

$\mathrm{Au}$ début de la section 2 on introduit une décomposition de $\widetilde{\mathfrak{g}}(\mathrm{F})$ en classes géométriques, dont on note l'ensemble $\mathcal{O}$, qui sont stables par action adjointe de $G(\mathrm{~F})$. Pour tous $\widetilde{P} \in \mathcal{F}\left(M_{\widetilde{0}}, B\right)$, $\mathfrak{o} \in \mathcal{O}$ et $f \in \mathcal{S}(\widetilde{\mathfrak{g}}(\mathbb{A}))$ on pose

$$
k_{f, \widetilde{P}, \mathfrak{o}}(x)=\sum_{\xi \in \operatorname{Lie}\left(M_{\widetilde{P}}\right)(\mathrm{F}) \cap \mathfrak{o}} \int_{\operatorname{Lie}\left(N_{\widetilde{P}}\right)(\mathbb{A})} f\left(x^{-1}(\xi+U) x\right) d U, x \in M_{\widetilde{P}}(\mathrm{~F}) N_{\widetilde{P}}(\mathbb{A}) \backslash \widetilde{G}(\mathbb{A}) .
$$

Le noyau tronqué est défini alors comme

$$
k_{f, \mathfrak{o}}^{T}(x)=\sum_{\widetilde{P} \in \mathcal{F}\left(M_{\widetilde{0}}, B\right)}(-1)^{d_{\widetilde{P}}-d_{\widetilde{G}}} \sum_{\delta \in P(\mathrm{~F}) \backslash G(\mathrm{~F})} \hat{\tau}_{\widetilde{P}}\left(H_{\widetilde{P}}(\delta x)-T\right) k_{f, \widetilde{P}, \mathfrak{o}}(\delta x), x \in \widetilde{G}(\mathrm{~F}) \backslash \widetilde{G}(\mathbb{A}),
$$

où, si l'on pose $\mathfrak{a}_{\widetilde{P}}:=\operatorname{Hom}_{\mathbb{Z}}\left(\operatorname{Hom}_{\mathrm{F}}\left(M_{\widetilde{P}}, \mathbb{G}_{m}\right), \mathbb{R}\right)$, alors $H_{\widetilde{P}}: \widetilde{G}(\mathbb{A}) \rightarrow \mathfrak{a}_{\widetilde{P}}$ c'est l'application de Harish-Chandra, $\hat{\tau}_{\widetilde{P}}$ est la fonction caractéristique d'un cône obtus dans $\mathfrak{a}_{\widetilde{P}}, d_{\widetilde{P}}=\operatorname{dim}_{\mathbb{R}} \mathfrak{a}_{\widetilde{P}}$ et $T$ est un paramètre dans $\mathfrak{a}_{\tilde{0}}:=\operatorname{Hom}_{\mathbb{Z}}\left(\operatorname{Hom}_{\mathrm{F}}\left(M_{\widetilde{0}}, \mathbb{G}_{m}\right), \mathbb{R}\right.$ ) (voir le paragraphe 1.1). Notre premier résultat, démontré dans le paragraphe 2.2 , c'est alors : 
Théoréme 0.1 (cf. théorème 2.6). Pour tout $T \in T_{+}+\mathfrak{a}_{\tilde{0}}^{+}$et tout $\sigma \in \mathbb{R}$ on a :

$$
\sum_{\mathfrak{o} \in \mathcal{O}} \int_{G(\mathrm{~F}) \backslash G(\mathbb{A})}\left|k_{f, \mathfrak{o}}^{T}(x)\right||\operatorname{det} x|_{\mathbb{A}}^{\sigma} d x<\infty .
$$

Ensuite, on s'intéresse au comportement de l'application $T \mapsto \int_{G(\mathrm{~F}) \backslash G(\mathbb{A})} k_{f, \mathfrak{o}}^{T}(x)|\operatorname{det} x|_{\mathbb{A}}^{s} \eta(\operatorname{det} x) d x$. Dans le paragraphe 3.3 on obtient :

Théoréme 0.2 (cf. théorème 3.8). Soit $\mathfrak{o} \in \mathcal{O}$ et $s \in \mathbb{C}$. La fonction

$$
T \mapsto I_{\mathfrak{o}}^{T}(s, f):=\int_{G(\mathrm{~F}) \backslash G(\mathbb{A})} k_{f, \mathfrak{o}}^{T}(x)|\operatorname{det} x|_{\mathbb{A}}^{s} \eta(\operatorname{det} x) d x,
$$

où $T$ parcourt $T_{+}+\mathfrak{a}_{\tilde{0}}^{+}$, est un polynôme-exponentielle dont la partie purement polynomiale est constante si $s \neq-1,1$.

On note $I_{\mathfrak{o}}(s, f)$ la partie constante du polynôme-exponentielle $I_{\mathfrak{o}}^{T}(s, f)$ pour $s \in \mathbb{C} \backslash\{-1,1\}$. Il s'avère que la distribution $I_{\mathfrak{o}}(s, \cdot)$ a des propriétés remarquables. On obtient :

Théoréme 0.3. Soient $f \in \mathcal{S}(\widetilde{\mathfrak{g}}(\mathbb{A}))$, o $\in \mathcal{O}$ et $s \in \mathbb{C} \backslash\{-1,1\}$.

(cf. théorème 3.11). Pour $y \in G(\mathbb{A})$, notons $\phi^{y} \in \mathcal{S}(\widetilde{\mathfrak{g}}(\mathbb{A}))$ définie par $\phi^{y}(X)=\phi(\operatorname{Ad}(y) X)$. On a alors

$$
I_{\mathfrak{o}}\left(s, f^{y}\right)=|\operatorname{det} y|_{\mathbb{A}}^{s} \eta(\operatorname{det} y) I_{\mathfrak{o}}(s, f) .
$$

(cf. paragraphe 3.5). La distribution $I_{\mathfrak{o}}(s, \cdot)$ ne dépendent que des choix des mesures de Haar.

Dans le paragraphe 3.6, on constate que pour les classes $\mathfrak{o}$ dites semi-simples régulières, sur lesquelles $G(\mathrm{~F})$ opère fidèlement et transitivement, la distribution $I_{\mathfrak{o}}^{T}(s, \cdot)$ ne dépend pas de $T$ et $I_{\mathfrak{o}}^{T}(s, f)$ égale une intégrale orbitale relative qui apparaît déjà dans [JR11].

Dans la section 4 on démontre la version infinitésimale de la formule des traces relative pour les groupes linéaires

Théoréme $\mathbf{0 . 4}$ (cf. théorème 4.1). Pour tout $f \in \mathcal{S}(\widetilde{\mathfrak{g}}(\mathbb{A}))$ et tout $s \in \mathbb{C} \backslash\{-1,1\}$ on a

$$
\sum_{\mathfrak{o} \in \mathcal{O}} I_{\mathfrak{o}}(s, f)=\sum_{\mathfrak{o} \in \mathcal{O}} I_{\mathfrak{o}}(s, \hat{f})
$$

Ici $\hat{f}$ c'est une transformée de Fourier (on en considère plusieurs) de $f$.

Notre dernier résultat, démontré dans la section 5 , ce sont des formules explicites pour certaines nouvelles distributions $I_{\mathfrak{o}}(s, \cdot)$. Tout $X \in \tilde{\mathfrak{g}}(\mathrm{F})$ s'écrit comme $\left(\begin{array}{ll}B & u \\ v & d\end{array}\right)$ où $B \in \operatorname{Lie}(G)(\mathrm{F})$, $u, v \in \mathrm{F}^{n}$ et $d \in \mathrm{F}$. On écrit alors $B_{X}:=B$. Soient $P \in \mathcal{F}(B)$ et $B \in \operatorname{Lie}\left(M_{P}\right)(\mathrm{F})$ un élément elliptique (qui n'est contenu dans aucun sous-algèbre parabolique propre de $\operatorname{Lie}\left(M_{P}\right)$ ). Soit $\mathfrak{o} \in \mathcal{O}$ une classe contenant un élément $X$ tel que $B_{X}=B$. Alors, o est une réunion finie d'orbites pour l'action de $G(\mathrm{~F})$. En plus, les orbites de dimension maximale dans $\mathfrak{o}$ ont des centralisateurs triviaux. Fixons un représentant $X_{0}$ de l'orbite fermée dans o tel que $B_{X_{0}}=B$ et choisissons un ensemble $\mathrm{O}_{\mathfrak{o}} \subseteq \mathfrak{o}$ de représentants des orbites de dimension maximale dans $\mathfrak{o}$ de sorte que tout $X \in \mathrm{O}_{\mathfrak{o}}$ vérifie $B_{X}=B$. Soit $T_{0}$ le centralisateur de $X_{0}$ dans $G$. Pour $f \in \mathcal{S}(\widetilde{\mathfrak{g}}(\mathbb{A}))$ et $X \in \mathrm{O}_{\mathfrak{o}}$ on pose :

$$
\zeta_{X}(f)(\lambda)=\int_{G(\mathbb{A})} f\left(\operatorname{Ad}\left(x^{-1}\right) X\right) e^{\lambda\left(H_{P}(x)\right)} \eta(\operatorname{det} x) d x
$$

où $\lambda \in \operatorname{Hom}_{\mathrm{F}}\left(T_{0}, \mathbb{G}_{m}\right) \otimes_{\mathbb{Z}} \mathbb{C}=: \mathfrak{a}_{\mathbb{C}}^{*}$ que l'on peut voir naturellement comme un sous-espace de $\operatorname{Hom}_{\mathbb{R}}\left(\mathfrak{a}_{P}, \mathbb{C}\right)$. L'intégrale définissant $\zeta_{X}(f)$ converge sur un ouvert de $\mathfrak{a}_{\mathbb{C}}^{*}$ qui dépend de $X$ et admet un prolongement méromorphe à $\mathfrak{a}_{\mathbb{C}}^{*}$ noté aussi $\zeta_{X}(f)$. Notons que le déterminant est naturellement un élément de $\operatorname{Hom}_{\mathrm{F}}\left(T_{0}, \mathbb{G}_{m}\right)$, on a donc un élément associé det $\in \mathfrak{a}_{\mathbb{C}}^{*}$. On obtient alors : 
Théoréme 0.5 (cf. théorème 5.12). Soit o comme ci-dessus. Alors, la droite $s$ det $\in \mathfrak{a}_{\mathbb{C}}^{*}$, où $s \in \mathbb{C}$, n'est contenu dans aucun hyperplan singulier de la fonction méromorphe

$$
\mathfrak{a}_{\mathbb{C}}^{*} \ni \lambda \mapsto\left(\sum_{X \in \mathrm{O}_{\mathfrak{o}}} \zeta_{X}(f)\right)(\lambda)
$$

En plus, la fonction méromorphe $\mathbb{C} \ni s \mapsto\left(\sum_{X \in \mathrm{O}_{\mathfrak{o}}} \zeta_{X}(f)\right)$ ( $s$ det) est holomorphe pour $s \neq-1,1$ et l'on $a$ :

$$
I_{\mathfrak{o}}(s, f)=\left(\sum_{X \in \mathrm{O}_{\mathfrak{o}}} \zeta_{X}(f)\right)(s \text { det }), \quad \forall s \in \mathbb{C} .
$$

Dans la section 5 de Zyd15a on obtient un résultat complètement analogue pour les groupes unitaires.

Commentons finalement l'apparition du déterminant à la puissance complexe dans la définition de la distribution $I_{\mathfrak{o}}(s, \cdot)$. On ajoute ce terme en vu de possibles applications aux dérivées des intégrales orbitales relatives de Jacquet-Rallis. Pour les classes semi-simples régulières, les dérivées des analogues locaux des distributions $I_{\mathfrak{o}}(s, \cdot)$ définies sur la variété $S_{n+1}$ ont d'abord été introduites dans [Zha12] et ensuite étudiées dans [RTZ13, ZRS15] pour des fonctions test particulières. Ces travaux lient ces dérivées à des nombres d'intersection sur certains espaces de Rapoport-Zink et s'inscrivent dans le cadre de la variante arithmétique de la conjecture de Gan-Gross-Prasad. Récemment, dans [Mih15], les dérivées des analogues locaux des distributions infinitésimales $I_{\mathfrak{o}}(s, \cdot)$ ont aussi été étudiées. On espère que notre formule va avoir un intérêt dans ces questions.

Remerciements. Je voudrais remercier mon directeur de thèse, Pierre-Henri Chaudouard, pour toute son aide.

\section{Prolégomènes}

\subsection{Préliminaires pour la formule des traces}

Soient $\mathrm{F}$ un corps de nombres et $G$ un F-groupe algébrique réductif que l'on suppose déployé sur F. Pour tout sous-F-groupe de Levi $M$ de $G$ (c'est-à-dire un facteur de Levi d'un F-sous-groupe parabolique de $G$ ) soit $\mathcal{F}(M)$ l'ensemble de F-sous-groupes paraboliques de $G$ contenant $M$ et $\mathcal{P}(M)$ le sous-ensemble de $\mathcal{F}(M)$ composé de sous-groupes paraboliques admettant $M$ comme facteur de Levi. On fixe un sous-groupe de Levi minimal $M_{0}$ de $G$. On appelle les éléments de $\mathcal{F}\left(M_{0}\right)$ les sous-groupes paraboliques semi-standards et les éléments de $\mathcal{P}\left(M_{0}\right)$ les sous-groupes de Borel. On utilisera toujours le symbole $P$, avec des indices éventuellement, pour noter un sous-groupe parabolique semi-standard. Pour tout $P \in \mathcal{F}\left(M_{0}\right)$ soit $N_{P}$ le radical unipotent de $P$ et $M_{P}$ le facteur de Levi de $P$ contenant $M_{0}$. On a alors $P=M_{P} N_{P}$. On note $A_{P}$ le tore central de $M_{P}$ déployé sur F et maximal pour cette propriété. Si $P$ est un sous-groupe de Borel, on a alors $A_{P}=M_{0}$ et on pose dans ce cas $A_{0}:=A_{P}=M_{P}$. Pour $P_{1} \in \mathcal{F}\left(M_{0}\right)$, quand il n'y aura pas d'ambiguïté, on écrit $N_{1}$ au lieu de $N_{P_{1}}, M_{1}$ au lieu de $M_{P_{1}}$ etc.

Soit $P \in \mathcal{F}\left(M_{0}\right)$. On définit le $\mathbb{R}$-espace vectoriel $\mathfrak{a}_{P}:=\operatorname{Hom}_{\mathbb{Z}}\left(\operatorname{Hom}_{\mathrm{F}}\left(M_{P}, \mathbb{G}_{m}\right), \mathbb{R}\right)$, isomorphe à $\operatorname{Hom}_{\mathbb{Z}}\left(\operatorname{Hom}_{\mathrm{F}}\left(A_{P}, \mathbb{G}_{m}\right), \mathbb{R}\right)$ grâce à l'inclusion $A_{P} \hookrightarrow M_{P}$, ainsi que son espace dual $\mathfrak{a}_{P}^{*}=\operatorname{Hom}_{\mathrm{F}}\left(M_{P}, \mathbb{G}_{m}\right) \otimes_{\mathbb{Z}} \mathbb{R}$ et on pose

$$
d_{P}=\operatorname{dim}_{\mathbb{R}} \mathfrak{a}_{P}, \quad d_{Q}^{P}=d_{Q}-d_{P}, Q \subseteq P .
$$

Si $P_{1} \subseteq P_{2}$, on a un homomorphisme injectif canonique $\mathfrak{a}_{2}^{*} \hookrightarrow \mathfrak{a}_{1}^{*}$ qui donne la projection $\mathfrak{a}_{1} \rightarrow \mathfrak{a}_{2}$, dont on note $\mathfrak{a}_{1}^{2}=\mathfrak{a}_{P_{2}}^{P_{2}}$ le noyau. On a aussi l'inclusion $\mathfrak{a}_{2} \hookrightarrow \mathfrak{a}_{1}$, qui est une section de 
$\mathfrak{a}_{1} \rightarrow \mathfrak{a}_{2}$, grâce à la restriction des caractères de $A_{1}$ à $A_{2}$. Il s'ensuit que si $P_{1} \subseteq P_{2}$ alors

$$
\mathfrak{a}_{1}=\mathfrak{a}_{1}^{2} \oplus \mathfrak{a}_{2} .
$$

Conformément à cette décomposition, on pose aussi $\left(\mathfrak{a}_{1}^{2}\right)^{*}=\left\{\lambda \in \mathfrak{a}_{1}^{*} \mid \lambda(H)=0 \forall H \in \mathfrak{a}_{2}\right\}$. On aura besoin aussi de $\left(\mathfrak{a}_{1, \mathbb{C}}^{2}\right)^{*}:=\left(\mathfrak{a}_{\widetilde{Q}}^{\widetilde{G}}\right)^{*} \otimes_{\mathbb{R}} \mathbb{C}$ et de $\mathfrak{a}_{1, \mathbb{C}}^{*}=\mathfrak{a}_{1}^{*} \otimes_{\mathbb{R}} \mathbb{C}$.

Si $P \subseteq Q$ sont des sous-groupes paraboliques semi-standards où $P$ est un sous-groupe de Borel on note simplement $\mathfrak{a}_{0}=\mathfrak{a}_{P}, \mathfrak{a}_{0}^{Q}=\mathfrak{a}_{P}^{Q}, \mathfrak{a}_{0}^{*}=\mathfrak{a}_{P}^{*}$ etc. Cela ne dépend pas du choix de $P$. En général donc, si $P_{1} \subseteq P_{2}$, grâce à la décomposition (1.2) ci-dessus, on considère les espaces $\mathfrak{a}_{1}$ et $\mathfrak{a}_{1}^{2}\left(\right.$ resp. $\mathfrak{a}_{1}^{*}$ et $\left.\left(\mathfrak{a}_{1}^{2}\right)^{*}\right)$ comme des sous-espaces de $\mathfrak{a}_{0}\left(\right.$ resp. $\left.\mathfrak{a}_{0}^{*}\right)$.

Notons $\Delta_{P}^{G}=\Delta_{P}$ l'ensemble de racines simples pour l'action de $A_{P}$ sur $N_{P}$. Il y a une correspondance bijective entre les sous-groupes paraboliques $P_{2}$ contenant $P_{1}$ et les sous-ensembles $\Delta_{1}^{2}=\Delta_{P_{1}}^{P_{2}}$ de $\Delta_{1}=\Delta_{P_{1}}$. En fait, $\Delta_{1}^{2}$ est l'ensemble de racines simples pour l'action de $A_{1}$ sur $N_{1} \cap M_{2}$ et l'on a

$$
\mathfrak{a}_{2}=\left\{H \in \mathfrak{a}_{1} \mid \alpha(H)=0 \forall \alpha \in \Delta_{1}^{2}\right\} .
$$

En plus $\Delta_{1}^{2}$ (les restrictions de ses éléments à $\left.\mathfrak{a}_{1}^{2}\right)$ est une base de $\left(\mathfrak{a}_{1}^{2}\right)^{*}$.

Fixons $P_{1} \subseteq P_{2}$ et soit $B \in \mathcal{P}\left(M_{0}\right)$ contenu dans $P_{1}$. On a alors l'ensemble $\Delta_{B}^{\vee}=\left\{\alpha^{\vee} \in\right.$ $\left.\mathfrak{a}_{0} \mid \alpha \in \Delta_{B}\right\}$ de coracines simples associées aux racines simples $\Delta_{0}$. Soit $\left(\Delta_{1}^{2}\right)^{\vee}$ l'ensemble de projections d'éléments de $\Delta_{B}^{\vee}$ à $\mathfrak{a}_{1}^{2}$ privé de zéro. Cela ne dépend pas du choix de $B$. L'ensemble $\Delta_{1}^{2}$ est en bijection canonique avec $\left(\Delta_{1}^{2}\right)^{\vee}$, la bijection étant : à $\alpha \in \Delta_{1}^{2}$ on associe l'unique $\alpha^{\vee} \in\left(\Delta_{1}^{2}\right)^{\vee}$ tel que $\alpha\left(\alpha^{\vee}\right)>0$. Notons également $\widehat{\Delta}_{1}^{2}$ et $\left(\widehat{\Delta}_{1}^{2}\right)^{\vee}$ les bases de $\left(\mathfrak{a}_{1}^{2}\right)^{*}$ et $\mathfrak{a}_{1}^{2}$ duales à $\left(\Delta_{1}^{2}\right)^{\vee}$ et $\Delta_{1}^{2}$ respectivement. Si $P_{2}=G$ on note simplement $\Delta_{1}, \Delta_{1}^{\vee}$ etc.

Soient $P, P_{1}, P_{2} \in \mathcal{F}\left(M_{0}\right)$, on note

$$
\mathfrak{a}_{P}^{+}=\left\{H \in \mathfrak{a}_{P} \mid \alpha(H)>0 \forall \alpha \in \Delta_{P}\right\}
$$

et si $P_{1} \subseteq P_{2}$ notons $\tau_{1}^{2}, \hat{\tau}_{1}^{2}$ les fonction caractéristiques de

$$
\left\{H \in \mathfrak{a}_{0} \mid \alpha(H)>0 \forall \alpha \in \Delta_{1}^{2}\right\}, \quad\left\{H \in \mathfrak{a}_{0} \mid \varpi(H)>0 \forall \varpi \in \widehat{\Delta}_{1}^{2}\right\}
$$

respectivement. On note $\tau_{P}$ pour $\tau_{P}^{G}$ et $\hat{\tau}_{P}$ pour $\hat{\tau}_{P}^{G}$

Soit $\mathbb{A}=\mathbb{A}_{\mathrm{F}}$ l'anneau des adèles de $\mathrm{F}$ et soit $|\cdot|_{\mathbb{A}}$ la valeur absolue standard sur le groupe des idèles $\mathbb{A}^{*}$. Pour tout $P \in \mathcal{F}\left(M_{0}\right)$, posons $H_{P}: M_{P}(\mathbb{A}) \rightarrow \mathfrak{a}_{P}$ défini comme

$$
\left\langle H_{P}(m), \chi\right\rangle=\log \left(|\chi(m)|_{\mathbb{A}}\right), \quad \chi \in \operatorname{Hom}_{\mathrm{F}}\left(M_{P}, \mathbb{G}_{m}\right), m \in M_{P}(\mathbb{A}) .
$$

C'est un homomorphisme continu et surjectif, donc si l'on note $M_{P}(\mathbb{A})^{1}$ son noyau, on obtient la suite exacte suivante

$$
1 \rightarrow M_{P}(\mathbb{A})^{1} \rightarrow M_{P}(\mathbb{A}) \rightarrow \mathfrak{a}_{P} \rightarrow 0 .
$$

Soit $A_{P}^{\infty}$ la composante neutre du groupe des $\mathbb{R}$-points du tore déployé et défini sur $\mathbb{Q}$ maximal pour cette propriété dans le $\mathbb{Q}$-tore $\operatorname{Res}_{\mathrm{F} / \mathbb{Q}} A_{P}$. Alors, comme $\mathrm{F} \otimes_{\mathbb{Q}} \mathbb{R}$ s'injecte dans $\mathbb{A}$, on a naturellement $A_{P}^{\infty} \hookrightarrow A_{P}(\mathbb{A}) \hookrightarrow M_{P}(\mathbb{A})$. En plus, la restriction de $H_{P}$ à $A_{P}^{\infty}$ est un isomorphisme donc $M_{P}(\mathbb{A})$ est un produit direct de $M_{P}(\mathbb{A})^{1}$ et $A_{P}^{\infty}$. Pour $Q \in \mathcal{F}\left(M_{0}\right)$ contenant $P$ on pose $A_{P}^{Q, \infty}=A_{P}^{\infty} \cap M_{Q}(\mathbb{A})^{1}$. L'application $H_{P}$ induit alors un isomorphisme entre $A_{P}^{Q, \infty}$ et $\mathfrak{a}_{P}^{Q}$.

Fixons $K$ un sous-groupe compact maximal admissible de $G(\mathbb{A})$ par rapport à $M_{0}$. La notion d'admissibilité par rapport à un sous-groupe de Levi minimal est définie dans le paragraphe 1 de Art81. On a donc, que pour tout sous-groupe parabolique semi-standard $P, K \cap M_{P}(\mathbb{A})$ est admissible dans $M_{P}(\mathbb{A})$ et on obtient aussi la décomposition d'Iwasawa $G(\mathbb{A})=P(\mathbb{A}) K=$ $N_{P}(\mathbb{A}) M_{P}(\mathbb{A}) K$ ce qui nous permet d'étendre $H_{P}$ à $G(\mathbb{A})$ en posant $H_{P}(x)=H_{P}(m)$ où $x=$ $n m k$ avec $m \in M_{P}(\mathbb{A}), n \in N_{P}(\mathbb{A}), k \in K$. Dans ce cas $H_{P}(x)$ ne dépend pas du choix de $m$.

On note $\Omega^{G}$ le groupe de Weyl de $\left(G, M_{0}\right)$. Pour tout $s \in \Omega^{G}$, on choisit un représentant $w_{s}$ dans l'intersection de $U(\mathrm{~F}) \cap K$ avec le normalisateur de $M_{0}$. Ceci n'est pas toujours possible 
donc on impose la condition sur $K$ que cela est possible. On peut toujours trouver un tel $K$ dans le cas où $G=\mathrm{GL}_{n}$ et c'est le cas qui va nous intéresser. Pour un F-sous-groupe $H$ de $G$ et $s \in \Omega^{G}$ on note $s H$ le F-sous-groupe $w_{s} H w_{s}^{-1}$. Le groupe $\Omega^{G}$ agit donc ainsi sur $\mathcal{F}\left(M_{0}\right)$. Pour tout $B \in \mathcal{P}\left(M_{0}\right)$ l'application $\Omega^{G} \ni s \mapsto s B \in \mathcal{P}\left(M_{0}\right)$ est une bijection. Pour tout $P \in \mathcal{F}\left(M_{0}\right)$ soit $\Omega^{P}$ le sous-groupe de $\Omega^{G}$ stabilisant $P$. On a donc $\Omega^{P}=\left\{s \in \Omega^{G} \mid w_{s} \in M_{P}(\mathrm{~F})\right\}$.

Notons finalement, que parfois, pour économiser l'espace, on utilisera la notation $[H]$ pour noter $H(\mathrm{~F}) \backslash H(\mathbb{A})$.

\subsection{Le domaine de Siegel}

Soit $B$ un sous-groupe de Borel de $G$ semi-standard. Pour un compact $\omega \subseteq N_{B}(\mathbb{A}) M_{0}(\mathbb{A})^{1}$ et un réel négatif $c$, on définit le domaine de Siegel $\mathfrak{S}_{B}(\omega, c)$ dans $G(\mathbb{A})$ comme :

$$
\mathfrak{S}_{B}(\omega, c)=\left\{m a k \in G(\mathbb{A}) \mid m \in \omega, k \in K, a \in A_{B}^{\infty}(c)\right\}
$$

où $A_{B}^{\infty}(c)=A_{B}^{\infty}(G, c)=\left\{a \in A_{B}^{\infty} \mid \alpha\left(H_{B}(a)\right)>c, \forall \alpha \in \Delta_{B}\right\}$. En général, pour un sous-groupe parabolique semi-standard $P$ de $G$ contenant $B$ on définit :

$$
\mathfrak{S}_{B}^{P}(\omega, c)=\left\{m a k \in G(\mathbb{A}) \mid m \in \omega, k \in K, a \in A_{B}^{\infty}(P, c)\right\} .
$$

où $A_{B}^{\infty}(P, c)=\left\{a \in A_{B}^{\infty} \mid \alpha\left(H_{B}(a)\right)>c, \forall \alpha \in \Delta_{B}^{P}\right\}$.

On utilisera le résultat suivant de la théorie de réduction, qu'on peut trouver, par exemple dans God64:

Proposition 1.1. Il existe un réel négatif $c_{0}$ et pour tout sous-groupe de Borel semi-standard $B$ de $G$ un compact $\omega_{B} \subseteq N_{B}(\mathbb{A}) M_{0}(\mathbb{A})^{1}$ tels que pour tout sous-groupe parabolique semi-standard $P$ contenant $B$ l'on a :

$$
G(\mathbb{A})=P(\mathrm{~F}) \mathfrak{S}_{B}^{P}\left(\omega_{B}, c_{0}\right) .
$$

Fixons la constante $c_{0}$ comme ci-dessus. Pour tout sous-groupe de Borel semi-standard $B$ on fixe aussi un $\omega_{B}$ comme dans la proposition 1.1 de façon que si $B^{\prime} \in \mathcal{P}\left(M_{0}\right)$ est tel que $s B=B^{\prime}$ on a $\omega_{B^{\prime}}=w_{s} \omega_{B} w_{s}^{-1}$. Les définitions de ce paragraphe sont valables en particulier pour les sousgroupes de Levi de $G$. On voit donc qu'on peut fixer un $\omega_{B}$ de façon que pour tout sous-groupe parabolique semi-standard $P$ et tout $B \in \mathcal{P}\left(M_{0}\right)$ le contenant le compact $M_{P}(\mathbb{A}) \cap \omega_{B}$ ainsi que le sous-groupe $K_{P}:=M_{P}(\mathbb{A}) \cap K$ jouissent des rôles de $\omega_{B}$ et $K$ ci-dessus par rapport au groupe réductif $M_{P}$ et son sous-groupe de Borel $B \cap M_{P}$, la constante $c_{0}$ restant la même.

Soient $B \in \mathcal{P}\left(M_{0}\right), P \supseteq B$ et $T \in \mathfrak{a}_{0}$. On définit $F_{B}^{P}(x, T)$ comme la fonction caractéristique de l'ensemble :

$$
\left\{x \in G(\mathbb{A}) \mid \exists \delta \in P(\mathrm{~F}) \delta x \in \mathfrak{S}_{B}^{P}\left(\omega_{B}, c_{0}\right), \varpi\left(H_{B}(\delta x)-T\right)<0 \forall \varpi \in \widehat{\Delta}_{B}^{P}\right\} .
$$

Autrement dit, si l'on pose

$$
A_{B}^{\infty}\left(P, c_{0}, T\right):=\left\{a \in A_{B}^{\infty}\left(P, c_{0}\right) \mid \varpi\left(H_{B}(a)-T\right)<0, \forall \varpi \in \widehat{\Delta}_{B}^{P}\right\},
$$

on a alors que $F_{B}^{P}(\cdot, T)$ est la fonction caractéristique de la projection de $\omega_{B} A_{B}^{\infty}\left(P, c_{0}, T\right) K$ sur $A_{P}^{\infty} N_{P}(\mathbb{A}) M_{P}(\mathrm{~F}) \backslash G(\mathbb{A})$.

\subsection{Algèbres de Lie}

Soit $\mathfrak{g}=\operatorname{Lie}(G)$, pour tout $P, P_{1}, P_{2} \in \mathcal{F}\left(M_{0}\right)$ tels que $P_{1} \subseteq P_{2}$ on note $N_{1}^{2}=N_{1} \cap M_{2}$, $\mathfrak{m}_{P}=\operatorname{Lie}\left(M_{P}\right), \mathfrak{n}_{P}=\operatorname{Lie}\left(N_{P}\right)$ et $\mathfrak{n}_{1}^{2}=\operatorname{Lie}\left(N_{1}^{2}\right)$. Soit $\bar{P} \in \mathcal{F}\left(M_{P}\right)$ le sous-groupe parabolique opposé à $P$ (i.e. tel que $\bar{P} \cap P=M_{P}$ ). On note alors $\overline{\mathfrak{n}}_{P}=\mathfrak{n}_{\bar{P}}$ et $\overline{\mathfrak{n}}_{1}^{2}=\mathfrak{m}_{2} \cap \overline{\mathfrak{n}}_{1}$.

On fixe une forme bilinéaire non-dégénérée $\langle\cdot, \cdot\rangle$ sur $\mathfrak{g}$ invariante par adjonction. Pour tout $P \in \mathcal{F}\left(M_{0}\right)$ la restriction de la forme $\langle\cdot, \cdot\rangle$ à $\overline{\mathfrak{n}}_{P} \times \mathfrak{n}_{P}$ est alors non-dégénérée, donc l'espace $\overline{\mathfrak{n}}_{P}$ s'identifie à l'espace dual à $\mathfrak{n}_{P}$ grâce à cette forme. 


\subsection{Fonctions de Bruhat-Schwartz}

On note $\mathbb{A}_{f}$ l'anneau des adèles finis de $\mathrm{F}$ et $\mathrm{F}_{\infty}:=\mathrm{F} \otimes_{\mathbb{Q}} \mathbb{R}$, de façon qu'on a $\mathbb{A}=\mathrm{F}_{\infty} \times \mathbb{A}_{f}$. Notons $\mathcal{S}(\mathfrak{g}(\mathbb{A}))$ l'ensemble des fonctions des Bruhat-Schwartz sur $\mathfrak{g}(\mathbb{A})$ i.e. l'espace de fonctions sur $\mathfrak{g}(\mathbb{A})$ engendré par des fonctions du type $f_{\infty} \otimes \chi^{\infty}$ où $f_{\infty}$ est une fonction de la classe de Schwartz sur $\mathfrak{g}\left(\mathrm{F}_{\infty}\right)$ et $\chi^{\infty}$ est une fonction caractéristique d'un compact ouvert de $\mathfrak{g}\left(\mathbb{A}_{f}\right)$.

\subsection{Les mesures de Haar}

Soit $P$ un sous-groupe parabolique semi-standard de $G$. On fixe $d x$ une mesure de Haar sur $G(\mathbb{A})$, ainsi que pour tout sous-groupe connexe $V$ de $N_{P}$ (resp. toute sous-algèbre $\mathfrak{h}$ de $\mathfrak{n}_{P}$ ) l'unique mesure de Haar sur $V(\mathbb{A})$ (resp. $\mathfrak{h}(\mathbb{A})$ ) pour laquelle le volume de $V(\mathrm{~F}) \backslash V(\mathbb{A})$ (resp. $\mathfrak{h}(\mathrm{F}) \backslash \mathfrak{h}(\mathbb{A}))$ soit 1 . Choisissons la mesure de Haar $d k$ sur K normalisé de même façon.

On fixe aussi une norme euclidienne $\|\cdot\|$ sur $\mathfrak{a}_{0}$ invariante par le groupe de Weyl $\Omega^{G}$ et sur tout sous-espace de $\mathfrak{a}_{0}$ la mesure de Haar compatible avec cette norme. Pour tout $Q \in \mathcal{F}\left(M_{0}\right)$ tel que $Q \supseteq P$, on en déduit les mesures de Haar sur $A_{P}^{Q, \infty}$ et $A_{P}^{\infty}$ via l'isomorphisme $H_{P}$.

Soit $d p$ la mesure de Haar sur $P(\mathbb{A})$ invariante à gauche normalisé de façon que $d x=d p d k$ (grâce à la décomposition d'Iwasawa). Notons $\rho_{P}^{G}=\rho_{P}$ l'élément de $\left(\mathfrak{a}_{P}^{G}\right)^{*}$ tel que $d\left(\operatorname{Ad}\left(m^{-1}\right) n\right)=$ $e^{2 \rho_{P}\left(H_{P}(m)\right)} d n$ pour $m \in M_{P}(\mathbb{A})$ et $n \in N_{P}(\mathbb{A})$. Il s'ensuit qu'il existe une unique mesure de Haar $d m$ sur $M_{P}(\mathbb{A})$ telle que si l'on écrit $p=n m$ où $p \in P(\mathbb{A}), n \in N_{P}(A)$ et $m \in M_{P}(\mathbb{A})$ alors $d p=e^{-2 \rho_{P}\left(H_{P}(m)\right)} d n d m$. Les mesures de Haar sur $M_{P}(\mathbb{A})$ et $A_{P}^{\infty}$ induisent alors une unique mesure de Haar sur $M_{P}(\mathbb{A})^{1}$, que l'on fixe, telle que la mesure de Haar sur $M_{P}(\mathbb{A})$ soit le produit de mesures sur $A_{P}^{\infty}$ et $\operatorname{sur} M_{P}(\mathbb{A})^{1}$.

\section{6 $\mathrm{GL}_{n} \hookrightarrow \mathrm{GL}_{n+1}$}

Soit $W$ un F-espace vectoriel de dimension finie $n+1$ et soit $V \subseteq W$ un sous-espace de dimension $n$, où $n \in \mathbb{N}$. Notons $\widetilde{G}=\mathrm{GL}(W)$. Fixons un vecteur $e_{0} \in W \backslash V$ et notons $D_{0}$ la droite qu'il engendre. On a alors $W=V \oplus D_{0}$ ce qui permet d'identifier $G=\mathrm{GL}(V)$ comme un sous-groupe de $\widetilde{G}$ stabilisant $V$ et fixant $e_{0}$. Choisissons $n$-droites $D_{1}, \ldots, D_{n}$ dans $V$ qui engendrent $V$. Soit $M_{0}$ le stabilisateur dans $G$ des droites $\left(D_{i}\right)_{i=1, \ldots, n}$. C'est un sous-F-groupe de Levi minimal de $G$. Soit alors $M_{\widetilde{0}}$ l'unique sous-F-groupe de Levi minimal de $\widetilde{G}$ contenant $M_{0}$. Alors $M_{\widetilde{0}}$ est le stabilisateur des droites $\left(D_{i}\right)_{i=0, \ldots, n}$ dans $\widetilde{G}$.

Les résultats des paragraphes précédentes s'appliquent aux groupes $G$ et $\widetilde{G}$ et leurs sousgroupes de Levi minimales $M_{0}$ et $M_{\widetilde{0}}$. Les objets associés à $\widetilde{G}$ seront notés toujours avec un tilde. Pour le choix du sous-groupe compact maximal, on fixe des vecteurs non-nuls $e_{i} \in D_{i}$ pour tout $i=1, \ldots, n$ ce qui avec le choix du vecteur $e_{0}$ défini les isomorphismes $\widetilde{G} \cong \mathrm{GL}_{n+1}$ et $G \cong \mathrm{GL}_{n}$. On pose alors $\widetilde{K}=\prod_{v} \widetilde{K}_{v}$ où, pour une place fini $v$ de $\mathrm{F}$ on note $\widetilde{K}_{v}=\mathrm{GL}_{n+1}\left(\mathcal{O}_{v}\right)$, où $\mathcal{O}_{v}$ c'est l'anneau des entiers de la complétion de F en $v$, pour une place réelle $v$ on pose $\widetilde{K}_{v}=O(n+1)$ le groupe orthogonal anisotrope et pour une place complexe on met $\widetilde{K}_{v}=U(n+1)$ - le groupe unitaire anisotrope. On pose aussi $K=\widetilde{K} \cap G(\mathbb{A})$. Dans ce cas $\widetilde{K}$ et $K$ vérifient les conditions du paragraphe (1.1). Les inclusions $G \hookrightarrow \widetilde{G}$ et $M_{0} \hookrightarrow M_{\widetilde{0}}$ induisent l'inclusion $\Omega^{G} \hookrightarrow \Omega^{\widetilde{G}}$. On choisit aussi des représentants du groupe de Weyl $\Omega^{\widetilde{G}}$ de $\widetilde{G}$ comme les éléments permutants les vecteurs $e_{i}$. On a alors pour tout $\widetilde{s} \in \Omega^{\widetilde{G}}$ que $w_{\widetilde{s}} \in \widetilde{G}(\mathrm{~F}) \cap \widetilde{K}$ et si $s \in \Omega^{G}$ alors $w_{s} \in G(\mathrm{~F}) \cap K$.

On identifie $\mathfrak{a}_{0}$ et $\mathfrak{a}_{0}^{*}$ avec des sous-espaces de $\mathfrak{a}_{\tilde{0}}$ et $\mathfrak{a}_{0}^{*}$ respectivement. En particulier la mesure de Haar et la norme euclidienne sur $\mathfrak{a}_{0}$ sont celles d'un sous-espace de $\mathfrak{a}_{\tilde{0}}$.

Posons $V_{i}=\oplus_{j=1}^{i} D_{i} \subseteq V$. On fixe $B \in \mathcal{P}\left(M_{0}\right)$ le stabilisateur du drapeau

$$
0 \subsetneq V_{1} \subsetneq \cdots \subsetneq V_{n}=V .
$$

On appelle $P \in \mathcal{F}\left(M_{0}\right)$ tels que $P \supseteq B$ les sous-groupes paraboliques standards de $G$. Tout $P \in \mathcal{F}\left(M_{0}\right)$ standard est alors défini comme le stabilisateur d'un sous-drapeau du drapeau (1.3) ci-dessus. 
Notons $V^{*}=\operatorname{Hom}_{\mathrm{F}}(V, \mathrm{~F})$ et $W^{*}=\operatorname{Hom}_{\mathrm{F}}(W, \mathrm{~F})$. Pour un F-sous-espace $\mathcal{V} \subseteq V$ de type $\mathcal{V}=\bigoplus_{i=k}^{l} D_{i}$ où $1 \leq k \leq l \leq n$ on pose $\mathcal{V}^{*}=\left\{\lambda \in V^{*}|\lambda|_{D_{i}}=0,1 \leq i<k, l<i \leq n\right\}$ et $\mathcal{V}^{\perp}=\left\{\lambda \in V^{*}|\lambda|_{\mathcal{V}}=0\right\}$

Pour tout $\widetilde{P} \in \mathcal{F}\left(M_{\widetilde{0}}\right)$ on admet la notation :

$$
P:=\widetilde{P} \cap G .
$$

Alors $P$ est un sous-groupe parabolique de $G$ semi-standard. Le groupe $G$ (resp. $\widetilde{G}$ ) agit aussi naturellement sur $V^{*}\left(\right.$ resp. $\left.W^{*}\right)$ donc aussi sur $V \times V^{*}\left(\right.$ resp. $\left.W \times W^{*}\right)$. Pour $\widetilde{P} \in \mathcal{F}\left(M_{\tilde{0}}\right)$ on note $\mathcal{V}_{\widetilde{P}} \subseteq V \times V^{*}$ le plus grand sous-espace de $V \times V^{*}$ stabilisé par $\widetilde{P}$ vu comme un sousespace de $W \times W^{*}$. On note aussi $Z_{\widetilde{P}} \subseteq V$ le plus petit sous-espace de $V$ tel que $M_{\widetilde{P}}$ stabilise $Z_{P} \oplus D_{0} \subseteq W$. On pose $\mathcal{Z}_{\widetilde{P}}=Z_{\widetilde{P}} \times Z_{\widetilde{P}}^{*} \subseteq V \times V^{*}$.

On note $\mathcal{F}\left(M_{\tilde{0}}, B\right)=\left\{P \in \mathcal{F}\left(M_{\widetilde{0}}\right) \mid B \subseteq \widetilde{P}\right\}$. On appelle les éléments de $\mathcal{F}\left(M_{\widetilde{0}}, B\right)$ les sous-groupes paraboliques relativement standards de $\widetilde{G}$.

Soit $\widetilde{P} \in \mathcal{F}\left(M_{\widetilde{0}}, B\right)$. Il est défini alors par une suite des entiers $0=i_{0} \leq \cdots \leq i_{l}=n$ et un entier $k$ tels que $1 \leq k \leq l \leq n+1$ et $k$ vérifie la propriété que si $i_{j-1}=i_{j}$ pour un $1 \leq j \leq l$ alors $j=k$. Le sous-groupe $\widetilde{P}$ est alors le stabilisateur du drapeau :

$$
0=V_{i_{0}} \subsetneq \cdots \subsetneq V_{i_{k-1}} \subsetneq V_{i_{k}} \oplus D_{0} \subsetneq \cdots \subsetneq V_{i_{l}} \oplus D_{0}=W .
$$

Dans ce cas $P$ est le stabilisateur de

$$
0=V_{i_{0}} \subsetneq \cdots \subsetneq V_{i_{k-1}} \subseteq V_{i_{k}} \subsetneq \cdots \subsetneq V_{i_{l}}=V .
$$

On a en plus

$$
\mathcal{V}_{\widetilde{P}}=V_{i_{k-1}} \times V_{i_{k}}^{\perp} \subseteq V \times V^{*}, \quad Z_{\widetilde{P}}=\bigoplus_{i_{k-1}<j \leq i_{k}} D_{i} .
$$

On voit qu'on a des isomorphismes :

$$
\begin{gathered}
M_{\widetilde{P}}=\prod_{1 \leq j<k} \mathrm{GL}_{i_{j}-i_{j-1}} \times \mathrm{GL}\left(Z_{\widetilde{P}} \oplus D_{0}\right) \times \prod_{k<j \leq l} \mathrm{GL}_{i_{j}-i_{j-1}} \cong \widetilde{G}_{\widetilde{P}} \times H_{\widetilde{P}} \\
M_{P}=\prod_{1 \leq j<k} \mathrm{GL}_{i_{j}-i_{j-1}} \times \mathrm{GL}\left(Z_{\widetilde{P}}\right) \times \prod_{k<j \leq l} \mathrm{GL}_{i_{j}-i_{j-1}} \cong G_{\widetilde{P}} \times H_{\widetilde{P}}
\end{gathered}
$$

où $\widetilde{G}_{\widetilde{P}}=\mathrm{GL}\left(Z_{\widetilde{P}} \oplus D_{0}\right), G_{\widetilde{P}}=\mathrm{GL}\left(Z_{\widetilde{P}}\right)$ et l'on voit $H_{\widetilde{P}}$ comme un sous-groupe de $M_{P}$, donc aussi de $M_{\widetilde{P}}$, qui agit trivialement $\operatorname{sur} Z_{\widetilde{P}}$. Dans ce contexte on note $A_{\widetilde{P}}^{s t, \infty}:=A_{H_{\widetilde{P}}}^{\infty}$ - c'est la partie standard du tore $A_{\widetilde{P}}^{\infty}$ puisque $A_{\widetilde{P}}^{\infty} \cap G(\mathbb{A})=A_{\widetilde{P}}^{s t, \infty}$, d'où la notation. On pose aussi $\mathfrak{a}_{\widetilde{P}}^{s t}:=\mathfrak{a}_{H_{\widetilde{P}}}$, donc $\mathfrak{a}_{\widetilde{P}}^{s t} \subseteq \mathfrak{a}_{\tilde{0}}$ ce qui détermine les mesures de Haar sur $\mathfrak{a}_{\widetilde{P}}^{s t}$ et sur $A_{\widetilde{P}}^{s t, \infty}$. On a alors :

$$
M_{P}(\mathbb{A})=A_{\widetilde{P}}^{s t, \infty}\left(H_{\widetilde{P}}(\mathbb{A})^{1} \times G_{\widetilde{P}}(\mathbb{A})\right), \quad M_{\widetilde{P}}(\mathbb{A})=A_{\widetilde{P}}^{s t, \infty}\left(H_{\widetilde{P}}(\mathbb{A})^{1} \times \widetilde{G}_{\widetilde{P}}(\mathbb{A})\right) .
$$

On fixe donc l'unique mesure de Haar sur $H_{\widetilde{P}}(\mathbb{A})^{1} \times G_{\widetilde{P}}(\mathbb{A})$ de façon que la mesure de Haar sur $M_{P}(\mathbb{A})$ choisie soit produit de cette mesure et celle sur $A_{\widetilde{P}}^{s t, \infty}$.

On fixe un $c_{0}<0$ tel que la proposition 1.1 ci-dessus soit vraie pour tous les groupes $\mathrm{GL}_{k}$ définis sur $F$ où $k \leq n+1$. On suppose alors que pour tout sous-groupe de Borel relativement standard $\widetilde{B}$ de $\widetilde{G}$ on a $\omega_{B} \subseteq \omega_{\widetilde{B}}$.

On remarque finalement une conséquence de notre choix des représentants du groupe de Weyl. On suppose donc que pour tout $\widetilde{s} \in \Omega^{\widetilde{G}}$ on a $w_{\widetilde{s}} \in \widetilde{G}(\mathrm{~F}) \cap \widetilde{K}$. Soient $\widetilde{s} \in \Omega^{\widetilde{G}}, \widetilde{B} \in \mathcal{P}\left(M_{\widetilde{0}}\right)$ et $\widetilde{P}, \widetilde{Q} \in \mathcal{F}\left(M_{\widetilde{0}}\right)$ tels que $\widetilde{P} \subseteq \widetilde{Q}$. Alors pour tout $x \in \widetilde{G}(\mathbb{A})$ et tout $T \in \mathfrak{a}_{\tilde{0}}$ les formules suivantes sont vraies :

$$
\begin{aligned}
\hat{\tau}_{\widetilde{s} \widetilde{P}}^{\widetilde{Q}}\left(H_{\widetilde{s} \widetilde{P}}(x)-T\right) & =\hat{\tau}_{\widetilde{P}}^{\widetilde{Q}}\left(H_{\widetilde{P}}\left(w_{\widetilde{s}}^{-1} x\right)-\widetilde{s}^{-1} T\right), \\
F_{\widetilde{s} B}^{\widetilde{s} P}(x, T) & =F_{\widetilde{B}}^{\widetilde{P}}\left(w_{\widetilde{s}}^{-1} x, \widetilde{s}^{-1} T\right) .
\end{aligned}
$$




\section{$1.7 \quad$ Relation entre $\mathfrak{a}_{\widetilde{P}}^{s t}$ et $\mathfrak{a}_{\widetilde{P}}$}

L'application naturelle $\mathfrak{a}_{\widetilde{P}}^{s t} \rightarrow \mathfrak{a}_{\widetilde{P}}^{\widetilde{G}}$, induit par la projection $\mathfrak{a}_{\widetilde{P}} \rightarrow \mathfrak{a}_{\widetilde{P}}^{\widetilde{G}}$, est un isomorphisme. On note $j_{\widetilde{P}}$ son Jacobien et on note $\iota_{\widetilde{P}}^{s t}:\left(\mathfrak{a}_{\widetilde{P}, \mathbb{C}}^{\widetilde{G}}\right)^{*} \rightarrow\left(\mathfrak{a}_{\widetilde{P}, \mathbb{C}}^{s t}\right)^{*}:=\operatorname{Hom}_{\mathbb{R}}\left(\mathfrak{a}_{\widetilde{P}}^{s t}, \mathbb{C}\right)=\mathfrak{a}_{H_{\widetilde{P}}, \mathbb{C}}^{*} l^{\prime}$ isomorphisme induit. Si $\widetilde{P}=\widetilde{G}$ on met $j_{\widetilde{P}}=1$.

Ainsi, pour toute fonction $\phi$ sur $\mathfrak{a}_{\widetilde{P}}$ qui est $\mathfrak{a}_{\widetilde{G}}$-invariante, et tout $\lambda \in\left(\mathfrak{a}_{\widetilde{P}, \mathbb{C}}^{s t}\right)^{*}$ on a :

$$
\int_{\mathfrak{a}_{\widetilde{P}}^{s t}} e^{\lambda(H)} \phi(H) d H=j_{\widetilde{P}}^{-1} \int_{\mathfrak{a}_{\widetilde{P}}} e^{l \stackrel{s t}{P}(\lambda)(H)} \phi(H) d H .
$$

\subsection{Quelques résultats de la théorie de la réduction}

On fixe un sous-groupe de Borel semi-standard $\widetilde{B}_{0} \in \mathcal{P}\left(M_{\widetilde{0}}\right)$ de $\widetilde{G}$. Pour tout $H \in \mathfrak{a}_{\widetilde{0}}$ et tout $\widetilde{P} \in \mathcal{F}\left(M_{\widetilde{0}}\right)$ on note $H_{\widetilde{P}} \in \mathfrak{a}_{\widetilde{P}}$ la projection de $\widetilde{s} H$ à $\mathfrak{a}_{\widetilde{P}}$ où $\widetilde{s} \in \Omega^{\widetilde{G}}$ est tel que $\widetilde{s} \widetilde{B}_{0} \subseteq \widetilde{P}$. Cette définition ne dépend pas du choix de $\widetilde{s}$.

La relation (1.10) nous permet de poser la définition suivante : soit $\widetilde{P} \in \mathcal{F}\left(M_{\widetilde{0}}\right)$ et $\widetilde{s} \in \Omega^{\widetilde{G}}$ tel que $\widetilde{s} \widetilde{B}_{0} \subseteq \widetilde{P}$, on pose donc :

$$
F^{\widetilde{P}}(x, T):=F_{\widetilde{s} \widetilde{B}_{0}}^{P}\left(x, T_{\widetilde{s} \widetilde{B}_{0}}\right), \quad x \in \widetilde{G}(\mathbb{A}), T \in \mathfrak{a}_{\widetilde{0}} .
$$

Cette définition ne dépend pas du choix de $\widetilde{s} \in \Omega^{\widetilde{G}}$ car $F_{\widetilde{B}}^{\widetilde{P}}$ est $M_{\widetilde{P}}(\mathrm{~F})$-invariant pour tout sous-groupe de Borel semi-standard $\widetilde{B}$ contenu dans $\widetilde{P}$. Remarquons aussi que si le paramètre $T$ appartient à la chambre $\mathfrak{a}_{\widetilde{B}_{0}}^{+}$alors $T_{\widetilde{s} \widetilde{B}_{0}}$ est dans la chambre $\mathfrak{a}_{\widetilde{s} \widetilde{B}_{0}}^{+}$. On note désormais :

$$
\mathfrak{a}_{\tilde{0}}^{+}:=\mathfrak{a}_{\widetilde{B}_{0}}^{+} .
$$

Le lemme suivant est l'application du lemme 6.4 de [Art78] à notre situation :

Lemme 1.2. Il existe un $T_{+} \in \mathfrak{a}_{0}^{+}$tel que pour tout sous-groupe parabolique semi-standard $\widetilde{Q}$ de $\widetilde{G}$, tout sous-groupe de Borel semi-standard $\widetilde{B}$ contenu dans $\widetilde{Q}$, tout $T \in T_{+}+\mathfrak{a}_{\widetilde{0}}^{+}$et tout $x \in \widetilde{G}(\mathbb{A})$ l'on $a:$

$$
\sum_{\widetilde{B} \subseteq \widetilde{P} \subseteq \widetilde{Q}} \sum_{\delta \in \widetilde{P}(\mathrm{~F}) \backslash \widetilde{Q}(\mathrm{~F})} F^{\widetilde{P}}(\delta x, T) \tau_{\widetilde{P}}^{\widetilde{Q}}\left(H_{\widetilde{P}}(\delta x)-T_{\widetilde{P}}\right)=1 .
$$

Fixons alors un $T_{+} \in \mathfrak{a}_{0}^{+}$comme dans le lemme 1.2 ci-dessus.

On a alors l'analogue relatif du lemme 1.2 .

Proposition 1.3. Soit $\widetilde{Q}$ un sous-groupe parabolique relativement standard de $\widetilde{G}$. Alors, pour tout $T \in T_{+}+\mathfrak{a}_{\tilde{0}}^{+}$et tout $x \in G(\mathbb{A})$, l'on $a$ :

$$
\sum_{B \subseteq \widetilde{P} \subseteq \widetilde{Q}} \sum_{\eta \in P(\mathrm{~F}) \backslash Q(\mathrm{~F})} F^{\widetilde{P}}(\eta x, T) \tau_{\widetilde{P}}^{\widetilde{Q}}\left(H_{\widetilde{P}}(\eta x)-T_{\widetilde{P}}\right)=1 .
$$

où la somme porte sur tous les sous-groupes paraboliques relativement standards de $\widetilde{G}$ contenus dans $\widetilde{Q}$ et on note $P=\widetilde{P} \cap G$ etc.

Démonstration. Soient $\widetilde{Q}$ et $T$ comme dans l'énoncé de la proposition. Fixons un sous-groupe de Borel relativement standard $\widetilde{B}$ contenu dans $\widetilde{Q}$. Pour un $\widetilde{P} \in \mathcal{F}\left(M_{\widetilde{0}}, B\right)$ tel que $\widetilde{B} \subseteq \widetilde{P} \subseteq \widetilde{Q}$. On fixe $\Omega_{\widetilde{P}, \widetilde{Q}}^{\widetilde{Q}}$ un ensemble de représentants dans $\left\{\widetilde{s} \in \Omega^{\widetilde{G}} \mid \widetilde{s}^{-1} \widetilde{P} \subseteq \widetilde{Q}\right.$ et $\left.\widetilde{s}^{-1} \widetilde{P} \in \mathcal{F}\left(M_{\widetilde{0}}, B\right)\right\}$ pour la relation $\widetilde{s}_{1} \sim \widetilde{s}_{2}$ si est seulement si $\widetilde{s}_{1} \widetilde{s}_{2}^{-1} \widetilde{P}=\widetilde{P}$. Pour $\widetilde{s} \in \Omega_{\widetilde{P}, \widetilde{Q}}^{\widetilde{G}}$ on note $P_{\widetilde{s}}=\left(\widetilde{s}^{-1} \widetilde{P}\right) \cap G$. 
On voit donc que la somme dans le lemme égale

$$
\sum_{\widetilde{B} \subseteq \widetilde{P} \subseteq \widetilde{Q}} \sum_{\widetilde{s} \in \Omega_{\widetilde{\widetilde{P}}, \widetilde{Q}}} \sum_{\eta \in P_{\widetilde{s}}(\mathrm{~F}) \backslash Q(\mathrm{~F})} F_{\widetilde{B}}^{\widetilde{\widetilde{P}}}\left(w_{\widetilde{s}} \eta x, T_{\widetilde{B}}\right) \tau_{\widetilde{P}}^{\widetilde{Q}}\left(H_{\widetilde{P}}\left(w_{\widetilde{s}} \eta x\right)-T_{\widetilde{P}}\right) .
$$

Sous cette forme le résultat est démontré dans [IY], lemme 2.3.

Soit $\widetilde{P}$ un sous-groupe parabolique relativement standard de $\widetilde{G}$. On note

$$
\mathcal{P}\left(M_{\widetilde{0}}, B, \widetilde{P}\right)=\left\{\widetilde{B} \in \mathcal{P}\left(M_{\widetilde{0}}\right) \cap \mathcal{F}\left(M_{\widetilde{0}}, B\right) \mid \widetilde{B} \subseteq \widetilde{P}\right\} .
$$

Posons $\omega_{P}=\omega_{B} \cap M_{P}(\mathbb{A})$ et $K_{P}=K \cap M_{P}(\mathbb{A})$. On a alors

Lemme 1.4. Soit $\widetilde{P} \in \mathcal{F}\left(M_{\widetilde{0}}, B\right)$. Le sous-ensemble suivant de $M_{P}(\mathbb{A})$

$$
\bigcup_{\widetilde{B} \in \mathcal{P}\left(M_{\widetilde{0}}, B, \widetilde{P}\right)} \omega_{P}\left(A_{B}^{\infty}\left(P, c_{0}\right) \cap A_{\widetilde{B}}^{\infty}\left(\widetilde{P}, c_{0}\right)\right) K_{P}
$$

se surjecte sur $M_{P}(\mathrm{~F}) \backslash M_{P}(\mathbb{A})$.

Démonstration. En vertu de la proposition 1.1 il suffit de montrer que pour tout $a \in A_{B}^{\infty}\left(P, c_{0}\right)$ il existe un $\widetilde{B} \in \mathcal{P}\left(M_{\widetilde{0}}, B, \widetilde{P}\right)$ tel que $a \in A_{\widetilde{B}}^{\infty}\left(\widetilde{P}, c_{0}\right)$. On se ramène facilement au cas $\widetilde{P}=\widetilde{G}$ et dans ce cas c'est l'assertion (2.5) de [IY].

On note aussitôt :

Corollaire 1.5. Soit $\widetilde{P} \in \mathcal{F}\left(M_{\widetilde{0}}, B\right)$. L'ensemble

$$
\bigcup_{\widetilde{B} \in \mathcal{P}\left(M_{\widetilde{0}}, B, \widetilde{P}\right)} \omega_{P}\left(A_{B}^{\infty}\left(P, c_{0}\right) \cap A_{\widetilde{B}}^{\infty}\left(\widetilde{P}, c_{0}, T_{\widetilde{B}}\right)\right) K_{P},
$$

où $\mathcal{P}\left(M_{\widetilde{0}}, B, \widetilde{P}\right)$ est défini par (1.13) ci-dessus, se surjecte sur l'ensemble des $m \in M_{P}(\mathrm{~F}) \backslash M_{P}(\mathbb{A})$ tels que $F^{\widetilde{P}}(m, T)=1$.

Démonstration. Soit $m \in M_{P}(\mathrm{~F}) \backslash M_{P}(\mathbb{A})$ et $\widetilde{B} \in \mathcal{P}\left(M_{\widetilde{0}}, B, \widetilde{P}\right)$ tel que $k_{1} a k_{2} \in \omega_{P}\left(A_{B}^{\infty}\left(P, c_{0}\right) \cap\right.$ $\left.A_{\widetilde{B}}^{\infty}\left(\widetilde{P}, c_{0}\right)\right) K_{P}$ est un représentant de $m$ dans $M_{P}(\mathbb{A})$, comme dans le lemme 1.4 ci-dessus. Il résulte du lemme 1.2 et du fait qu'il existe une constante $c$ telle que $\varpi\left(H_{\widetilde{B}}\left(w_{\widetilde{s}} n\right)\right) \leq c$ pour tout $\varpi \in \widehat{\Delta}_{\widetilde{B}}$ et tout $\widetilde{s} \in \Omega^{\widetilde{G}}$ que si $\widetilde{k}_{1} \in \omega_{\widetilde{B}} \cap M_{\widetilde{P}}(\mathbb{A}), \widetilde{a} \in A_{\widetilde{B}}^{\infty}\left(\widetilde{P}, c_{0}\right)$ et $\widetilde{k} \in \widetilde{K} \cap M_{\widetilde{P}}(\mathbb{A})$ sont tels que $F_{\widetilde{B}} \widetilde{P}\left(\widetilde{k}_{1} \widetilde{a} \widetilde{k}, T\right)=1$ alors $F_{\widetilde{B}} \widetilde{P}(\widetilde{a}, T)=1$. En particulier, puisque $\omega_{B} \subseteq \omega_{\widetilde{B}}$ pour tout $\widetilde{B} \in \mathcal{F}\left(M_{\widetilde{0}}, B\right) \cap \mathcal{P}\left(M_{\widetilde{0}}\right)$ et $K \subseteq \widetilde{K}$, si l'on suppose que $F^{\widetilde{P}}(m, T)=F_{\widetilde{B}}^{\widetilde{P}}\left(k_{1} a k_{2}, T_{\widetilde{B}}\right)=1$ on a $F_{\widetilde{B}}^{\widetilde{P}}\left(a, T_{\widetilde{B}}\right)=1$ d'où $a \in A_{B}^{\infty}\left(P, c_{0}\right) \cap A_{\widetilde{B}}^{\infty}\left(\widetilde{P}, c_{0}\right)$ et le corollaire suit.

\subsection{Majorations cruciales}

Dans ce paragraphe on se propose de donner une majoration de l'intégrale du type :

$$
\int_{M_{P}(\mathrm{~F}) \backslash M_{P}(\mathbb{A})} e^{\rho\left(H_{P}(m)\right)} F^{\widetilde{P}}(m, T)|\Phi(m)| d m
$$

où $\widetilde{P} \in \mathcal{F}\left(M_{\widetilde{0}}, B\right), \rho \in \mathfrak{a}_{P}^{*}$, et $\Phi$ est une fonction sur $M_{\widetilde{P}}(\mathbb{A})$ invariante à gauche par $M_{\widetilde{P}}(\mathrm{~F}) A_{\widetilde{G}}^{\infty}$. Pour $\widetilde{B} \in \mathcal{P}\left(M_{\widetilde{0}}, B, \widetilde{P}\right)$ notons :

$$
A_{\widetilde{B}}^{\widetilde{G}, \infty}\left(\widetilde{P}, c_{0}, T_{\widetilde{B}}\right):=A_{\widetilde{B}}^{\infty}\left(\widetilde{P}, c_{0}, T_{\widetilde{B}}\right) \cap A_{\widetilde{B}}^{\widetilde{G}, \infty}
$$

On a alors : 
Lemme 1.6. Il existe un $c>0$ tel que pour toute fonction $\Phi$ comme ci-dessus, tout $T \in T_{+}+\mathfrak{a}_{\tilde{0}}^{+}$, tout $\rho \in \mathfrak{a}_{P}^{*}$ et tout $\widetilde{B} \in \mathcal{P}\left(M_{\widetilde{0}}, B, \widetilde{P}\right)$ il existe des $\rho_{\widetilde{B}, \widetilde{P}} \in\left(\mathfrak{a}_{\widetilde{B}}^{\widetilde{G}}\right)^{*}$ tels que l'intégrale 1.14) est majorée par c fois

$$
\sum_{\widetilde{B} \in \mathcal{P}\left(M_{\widetilde{0}}, B, \widetilde{P}\right)} \int_{A_{\widetilde{B}}^{\widetilde{\widetilde{G}}, \infty}\left(\widetilde{P}, c_{0}, T_{\widetilde{B}}\right)} e^{\rho_{\widetilde{B}, \widetilde{P}}\left(H_{\widetilde{B}}(a)\right)} \sup _{\substack{k_{1} \in \omega_{\widetilde{B}} \cap M_{\widetilde{P}}(\mathbb{A}) \\ k_{2} \in \widetilde{K} \cap M_{\widetilde{P}}(\mathbb{A})}}\left|\Phi\left(k_{1} a k_{2}\right)\right| d a .
$$

Démonstration. Soient $B_{P}=B \cap M_{P}$ et $B_{P}(\mathbb{A})^{1}=N_{B}^{P}(\mathbb{A}) M_{B}(\mathbb{A})^{1}$. Alors $\omega_{P} \subseteq B_{P}(\mathbb{A})^{1}$. Le compact $K_{P}$ est un sous-groupe compact maximal de $M_{P}(\mathbb{A})$. La décomposition d'Iwasawa implique qu'on a $M_{P}(\mathbb{A})=B_{P}(\mathbb{A})^{1} A_{B}^{\infty} K_{P}$. Il existe alors une unique mesure $d b^{\prime}$ sur $B_{P}(\mathbb{A})^{1}$ invariante à gauche telle que $d m=e^{-2 \rho_{B}^{P\left(H_{P}(a)\right)}} d b^{\prime} d a d k$.

En utilisant le corollaire 1.5 on voit alors que l'intégrale (1.14) est majorée par :

$$
\sum_{\widetilde{B} \in \mathcal{P}\left(M_{\widetilde{0}}, B, \widetilde{P}\right)} \operatorname{vol}\left(\omega_{P}\right) \quad \int_{A_{B}^{\infty}\left(P, c_{0}\right) \cap A_{\widetilde{B}}^{\infty}\left(\widetilde{P}, c_{0}, T_{\widetilde{B}}\right)} e^{\left(\rho-2 \rho_{B}^{P}\right)\left(H_{B}(a)\right)} \sup _{\substack{k_{1} \in \omega_{P} \\ k_{2} \in K_{P}}}\left|\Phi\left(k_{1} a k_{2}\right)\right| d a .
$$

Soit $\widetilde{B} \in \mathcal{P}\left(M_{\widetilde{0}}, B, \widetilde{P}\right)$. On peut appliquer les résultats du paragraphe 1.7 à $\widetilde{P}=\widetilde{B}$ car on a $\mathfrak{a}_{B}=\mathfrak{a}_{\widetilde{B}}^{s t}$. Si l'on note $\rho_{\widetilde{P}, \widetilde{B}}=\iota_{\widetilde{B}}^{s t}\left(\rho-2 \rho_{B}^{P}\right) \in\left(\mathfrak{a}_{\widetilde{B}}^{\widetilde{G}}\right)^{*}$, on obtient, en vertu de l'égalité (1.11), que (1.15) égale

$$
\sum_{\widetilde{B} \in \mathcal{P}\left(M_{\widetilde{0}}, B, \widetilde{P}\right)} j_{\widetilde{B}}^{-1} \operatorname{vol}\left(\omega_{P}\right) \quad \int_{A_{\widetilde{B}}^{\widetilde{\widetilde{P}}}\left(\widetilde{P}_{,}, c_{0}, T_{\widetilde{B}}\right)} e^{\rho_{\widetilde{B}, \widetilde{P}}\left(H_{\widetilde{B}}(a)\right)} \sup _{\substack{k_{1} \in \omega_{P} \\ k_{2} \in K_{P}}}\left|\Phi\left(k_{1} a k_{2}\right)\right| d a .
$$

On conclut en remarquant que $\omega_{P} \subseteq \omega_{\widetilde{B}} \cap M_{\widetilde{P}}(\mathbb{A})$ et $K_{P} \subseteq \widetilde{K} \cap M_{\widetilde{P}}(\mathbb{A})$.

\section{Formule des traces relative pour $\mathfrak{g l}(n+1) / / \mathrm{GL}(n)$}

On note $\mathfrak{g}=\operatorname{Lie}(G)$ et $\widetilde{\mathfrak{g}}=\operatorname{Lie}(\widetilde{G})$. Conformément au paragraphe 1.3 , pour tout $\widetilde{P} \in \mathcal{F}\left(M_{\widetilde{0}}\right)$, on note $\mathfrak{m}_{\widetilde{P}}=\operatorname{Lie}\left(M_{\widetilde{P}}\right), \mathfrak{n}_{\widetilde{P}}=\operatorname{Lie}\left(N_{\widetilde{P}}\right)$, etc. Pour la forme bilinéaire $\langle\cdot, \cdot\rangle$ on choisit la forme trace.

\subsection{Les invariants}

Soit $X \in \widetilde{\mathfrak{g}}$, suivant la décomposition $W=V \oplus D_{0}$ on écrit :

$$
X=\left(\begin{array}{ll}
B & u \\
v & d
\end{array}\right)
$$

où $B \in \mathfrak{g}, u \in \operatorname{Hom}_{\mathrm{F}}\left(D_{0}, V\right), v \in \operatorname{Hom}_{\mathrm{F}}\left(V, D_{0}\right)$ et $d \in \operatorname{Hom}_{\mathrm{F}}\left(D_{0}, D_{0}\right)$. On rappelle qu'on a fixé un vecteur non-nul $e_{0} \in D_{0}$. On note alors $e_{0}^{*} \in W^{*}$ défini par $e_{0}^{*}\left(e_{0}\right)=1$ et $\left.e_{0}^{*}\right|_{V} \equiv 0$ et on identifie donc $d$ avec $e_{0}^{*}\left(d\left(e_{0}\right)\right) \in \mathbb{G}_{a}, u$ avec $u\left(e_{0}\right) \in V$ et $v$ avec l'élément de $V^{*}=\operatorname{Hom}_{\mathrm{F}}(V, \mathrm{~F})$ défini par $x \mapsto e_{0}^{*}(v(x))$.

On dit que $X \in \tilde{\mathfrak{g}}$ est semi-simple régulier s'il vérifie les conditions de la proposition suivante, due à [SG08], théorème 6.1 et proposition 6.3.

Proposition 2.1. Soit $X=\left(\begin{array}{ll}B & u \\ v & d\end{array}\right) \in \widetilde{\mathfrak{g}}$, alors les conditions suivantes sont équivalentes :

1) $\operatorname{det}\left(a_{i j}\right) \neq 0$ où $a_{i j}=v B^{i+j} u, 0 \leq i, j \leq n-1$.

2) Le stabilisateur de $X$ dans $G$ est trivial et l'orbite de $X$ dans $\widetilde{\mathfrak{g}}$ pour l'action de $G$ est fermée pour la topologie de Zariski. 
On introduit alors les invariants suivants pour l'action de $G$ sur $\tilde{\mathfrak{g}}$. Soit $X=\left(\begin{array}{ll}B & u \\ v & d\end{array}\right) \in$ $\widetilde{\mathfrak{g}}$ comme ci-dessus. On pose $A_{0}(X)=d$ et $A_{i}(X)=v B^{i-1} u$ pour $i=1,2, \ldots, n$ ainsi que $B_{j}(X)=\operatorname{Tr} \bigwedge^{j} B$ pour $j=1, \ldots, n$. Alors, le lemme 3.1 de [Zha14b] dit que les fonctions $A_{i}$, $B_{j}$ engendrent l'anneau des polynômes sur $\widetilde{\mathfrak{g}}$ invariants sous l'action de $G$. Ils définissent alors une relation d'équivalence sur $\widetilde{\mathfrak{g}}(\mathrm{F})$, moins fine que la relation de conjugaison par $G(\mathrm{~F})$, où $X, Y \in \tilde{\mathfrak{g}}(\mathrm{F})$ sont dans la même classe si et seulement si $A_{i}(X)=A_{i}(Y)$ et $B_{j}(X)=B_{j}(Y)$ pour $i=0, \ldots, n$ et $j=1, \ldots, n$. Notons $\mathcal{O}$ l'ensemble des classes d'équivalence pour cette relation. Cette relation pour des éléments semi-simples réguliers coïncide avec la relation de conjugaison sous $G(\mathrm{~F})$ comme il est démontrée dans [SG08], proposition 6.2.

Lemme 2.2. Soient $X$ et $Y$ deux éléments semi-simples réguliers de $\widetilde{\mathfrak{g}}(\mathrm{F})$. Ils appartiennent à la même classe dans $\mathcal{O}$ si et seulement s'ils sont conjugués par $G(\mathrm{~F})$.

Dans le paragraphe 1.6. on a introduit pour tout $\widetilde{P} \in \mathcal{F}\left(M_{\widetilde{0}}\right)$ des sous-espaces $\mathcal{V}_{\widetilde{P}}$ et $\mathcal{Z}_{\widetilde{P}}$ de $V \times V^{*}$. Voici leur rapport avec la décomposition (2.1).

Lemme 2.3. Soient $X=\left(\begin{array}{ll}B & u \\ v & d\end{array}\right) \in \widetilde{\mathfrak{g}}$ et $\widetilde{P} \in \mathcal{F}\left(M_{\widetilde{0}}\right)$ Alors

i) $X \in \mathfrak{m}_{\widetilde{P}}$ si et seulement si $B \in \mathfrak{m}_{P}$ et $(u, v) \in \mathcal{Z}_{\widetilde{P}}$.

ii) $X \in \mathfrak{n}_{\widetilde{P}}$ si et seulement si $B \in \mathfrak{n}_{P}$ et $(u, v) \in \mathcal{V}_{\widetilde{P}}$.

Démonstration. Il s'agit d'un calcul matriciel direct.

On étudiera maintenant les intersections des classes $\mathfrak{o} \in \mathcal{O}$ avec les algèbres de Lie de sousgroupes paraboliques relativement standards.

Proposition 2.4. Soit $\widetilde{P}$ un sous-groupe parabolique relativement standard de $\widetilde{G}$. Alors, pour tous $X \in \mathfrak{m}_{\widetilde{P}}(\mathrm{~F}), N \in \mathfrak{n}_{\widetilde{P}}(\mathrm{~F})$ et $\mathfrak{o} \in \mathcal{O}$ on $a:$

$$
X \in \mathfrak{o} \Longleftrightarrow X+N \in \mathfrak{o} .
$$

Démonstration. La preuve s'appuie sur le lemme 2.3 ci-dessus et est essentiellement identique à celle de la proposition 1.5 de Zyd15a.

Corollaire 2.5. Soient $\widetilde{P} \in \mathcal{F}\left(M_{\widetilde{0}}, B\right)$ et $\mathfrak{o} \in \mathcal{O}$. Alors pour tout $A \subseteq \mathfrak{m}_{\widetilde{P}}(\mathrm{~F})$ et tout $B \subseteq \mathfrak{n}_{\widetilde{P}}(\mathrm{~F})$ on $a$ :

$$
\mathfrak{o} \cap(A \oplus B)=(\mathfrak{o} \cap A) \oplus B .
$$

\subsection{Convergence du noyau modifié}

On définit det $\in \mathfrak{a}_{\tilde{0}}^{*}$ comme le déterminant du tore $A_{\widetilde{0}}$ pour son action sur $W$. Notons que pour tout $x \in G(\mathbb{A})$ et tout $\sigma \in \mathbb{R}$ on a alors $|\operatorname{det} x|_{\mathbb{A}}^{\sigma}=e^{\sigma \operatorname{det}\left(H_{G}(x)\right)}$.

Soit $f \in \mathcal{S}(\widetilde{\mathfrak{g}}(\mathbb{A}))$. Pour tout $\widetilde{P} \in \mathcal{F}\left(M_{\widetilde{0}}, B\right)$ et toute classe $\mathfrak{o} \in \mathcal{O}$ posons

$$
k_{\widetilde{P}, \mathfrak{o}}(x)=k_{f, \widetilde{P}, \mathfrak{o}}(x)=\sum_{\xi \in \mathfrak{m}_{\widetilde{P}}(\mathrm{~F}) \cap \mathfrak{o}} \int_{\mathfrak{n}_{\widetilde{P}}(\mathbb{A})} f\left(x^{-1}\left(\xi+U_{\widetilde{P}}\right) x\right) d U_{\widetilde{P}}, x \in M_{P}(\mathrm{~F}) N_{P}(\mathbb{A}) \backslash G(\mathbb{A})
$$

Pour $T \in \mathfrak{a}_{\tilde{0}}$ on pose alors

$$
k_{\mathfrak{o}}^{T}(x)=k_{f, \mathfrak{o}}^{T}(x)=\sum_{\widetilde{P} \in \mathcal{F}\left(M_{\widetilde{0}}, B\right)}(-1)^{d d_{\widetilde{P}}^{\widetilde{G}}} \sum_{\delta \in P(\mathrm{~F}) \backslash G(\mathrm{~F})} \hat{\tau}_{\widetilde{P}}\left(H_{\widetilde{P}}(\delta x)-T_{\widetilde{P}}\right) k_{\widetilde{P}, \mathfrak{o}}(\delta x), x \in G(\mathrm{~F}) \backslash G(\mathbb{A})
$$

où $d \widetilde{\widetilde{G}}$ est défini par (1.1). N.B. la somme sur $\delta$ dans $P(\mathrm{~F}) \backslash G(\mathrm{~F})$ est finie en vertu du lemme 5.1 de Art78. 
Théoréme 2.6. On a pour tout $T \in T_{+}+\mathfrak{a}_{\tilde{0}}^{+}$et tout $\sigma \in \mathbb{R}$

$$
\sum_{\mathfrak{o} \in \mathcal{O}} \int_{G(\mathrm{~F}) \backslash G(\mathbb{A})}\left|k_{\mathfrak{o}}^{T}(x)\right||\operatorname{det} x|_{\mathbb{A}}^{\sigma} d x<\infty
$$

Démonstration. En utilisant la proposition (1.3) on a que $k_{\mathfrak{o}}^{T}(x)$ égale la somme sur $\widetilde{P} \in$ $\mathcal{F}\left(M_{\widetilde{0}}, B\right)$ de $(-1)^{d \widetilde{\widetilde{G}}}$ fois la somme sur $\delta$ dans $P(\mathrm{~F}) \backslash G(\mathrm{~F})$ de

$$
\left(\sum_{\mathcal{F}\left(M_{\widetilde{0}}, B\right) \ni \widetilde{P}_{1} \subseteq \widetilde{P}} \sum_{\eta \in P_{1}(\mathrm{~F}) \backslash P(\mathrm{~F})} F^{\widetilde{P}_{1}}\left(\eta \delta x, T_{\widetilde{P}_{1}}\right) \tau_{\widetilde{P}_{1}}^{\widetilde{P}_{1}}\left(H_{\widetilde{P}_{1}}(\eta \delta x)-T_{\widetilde{P}_{1}}\right)\right) \hat{\tau}_{\widetilde{P}}\left(H_{\widetilde{P}}(\delta x)-T_{\widetilde{P}}\right) k_{\widetilde{P}, \mathfrak{o}}(\delta x) .
$$

Suivant l'article [Art78], les paragraphes 6 et 7 , on a :

$$
\tau_{\widetilde{P}_{1}}^{\widetilde{P}}(H) \hat{\tau}_{\widetilde{P}}(H)=\sum_{\widetilde{P}_{2} \supseteq \widetilde{P}} \sigma_{\widetilde{1}}^{\widetilde{2}}(H) \quad H \in \mathfrak{a}_{\widetilde{1}}
$$

où

$$
\sigma_{\widetilde{P}_{1}}^{\widetilde{P}_{2}}(H)=\sigma_{\widetilde{1}}^{\widetilde{2}}(H)=\sum_{\widetilde{Q} \supseteq \widetilde{P}_{2}}(-1)^{d_{2}^{\widetilde{Q}}} \tau_{\widetilde{P}_{1}}^{\widetilde{Q}}(H) \hat{\tau}_{\widetilde{Q}}(H), H \in \mathfrak{a}_{\tilde{1}} .
$$

Si l'on pose alors

$$
\begin{aligned}
& \chi_{\widetilde{1}, \widetilde{2}}^{T}(x)=F^{\widetilde{1}}(x, T) \sigma_{\widetilde{1}}^{\widetilde{2}}\left(H_{\widetilde{1}}(x)-T_{\widetilde{1}}\right), x \in \widetilde{P}_{1}(\mathrm{~F}) \backslash \widetilde{G}(\mathbb{A}), \\
& k_{\widetilde{P}_{1}, \widetilde{P}_{2}, \mathfrak{o}}(x)=k_{\widetilde{1}, \widetilde{2}, \mathfrak{o}}(x)=\sum_{\widetilde{P}_{1} \subseteq \widetilde{P} \subseteq \widetilde{P}_{2}}(-1)^{d d_{\widetilde{P}}^{\widetilde{\widetilde{P}}}} k_{\widetilde{P}, \mathfrak{o}}(x), x \in P_{1}(\mathrm{~F}) \backslash G(\mathbb{A}),
\end{aligned}
$$

on s'aperçoit alors que

$$
k_{\mathfrak{o}}^{T}(x)=\sum_{\widetilde{P}_{1} \subseteq \widetilde{P}_{2}} \sum_{\delta \in P_{1}(\mathrm{~F}) \backslash G(\mathrm{~F})} \chi_{\widetilde{1}, \widetilde{2}}^{T}(\delta x) k_{\widetilde{1}, \widetilde{2}, \mathfrak{o}}(\delta x)
$$

où la somme porte sur tous les couples $\widetilde{P}_{1}, \widetilde{P}_{2} \in \mathcal{F}\left(M_{\widetilde{0}}, B\right)$ tels que $\widetilde{P}_{1} \subseteq \widetilde{P}_{2}$.

On fixe alors de tels $\widetilde{P}_{1} \subseteq \widetilde{P}_{2}$ et l'on s'aperçoit qu'il suffit de démontrer :

$$
\sum_{\mathfrak{o} \in \mathcal{O}} \int_{P_{1}(\mathrm{~F}) \backslash G(\mathbb{A})} \chi_{\widetilde{1}, \widetilde{2}}^{T}(x)\left|k_{\widetilde{1}, \widetilde{2}, \mathfrak{o}}(x)\right| e^{\sigma \operatorname{det}\left(H_{G}(x)\right)} d x<\infty .
$$

Si $\widetilde{P}_{1}=\widetilde{P}_{2} \neq \widetilde{G}$, c'est une conséquence du lemme 5.1 de Art78] que $\sigma_{\widetilde{1}}^{\widetilde{2}} \equiv 0$ donc l'intégrale (2.7) converge. Si $\widetilde{P}_{1}=\widetilde{P}_{2}=\widetilde{G}$ les résultats de la section [1.9, le lemme 1.6 en l'occurrence, montrent que l'intégrale (2.7) est majorée par une somme sur $\widetilde{B} \in \mathcal{F}\left(M_{\widetilde{0}}, B\right) \cap \mathcal{P}\left(M_{\widetilde{0}}\right)$ d'une intégrale d'une fonction continue sur un ensemble $A_{\widetilde{B}}^{\widetilde{G}, \infty}\left(\widetilde{G}, c_{0}, T_{\widetilde{B}}\right)$ qui est compact donc on a aussi la convergence dans ce cas. Si $\widetilde{P}_{1} \subsetneq \widetilde{P}_{2}$ on démontrera un peu plus :

Théoréme 2.7. Soient $f \in \mathcal{S}(\widetilde{\mathfrak{g}}(\mathbb{A})), \sigma \in \mathbb{R}$ et $\widetilde{P}_{1}, \widetilde{P}_{2}$ deux sous-groupes paraboliques relativement standards de $\widetilde{G}$ tels que $\widetilde{P}_{1} \subsetneq \widetilde{P}_{2}$. Alors pour tout réel $\varepsilon_{0}>0$ et tout $N \in \mathbb{N}$ il existe une constante $C$ qui ne dépend que de $N, f, \sigma$ et $\varepsilon_{0}$ telle que:

$$
\sum_{\mathfrak{o} \in \mathcal{O}} \int_{P_{1}(\mathrm{~F}) \backslash G(\mathbb{A})} \chi_{\widetilde{1}, \widetilde{2}}^{T}(x)\left|k_{\widetilde{1}, \widetilde{2}, \mathfrak{o}}(x) \| \operatorname{det} x\right|_{\mathbb{A}}^{\sigma} d x<C e^{-N\|T\|}
$$

pour tout $T \in T_{+}+\mathfrak{a}_{\tilde{0}}^{+}$tels que $\forall \alpha \in \Delta_{\widetilde{B}_{0}} \alpha(T)>\varepsilon_{0}\|T\|$. 
On fixe alors deux sous-groupes relativement standards $\widetilde{P}_{1} \subsetneq \widetilde{P}_{2}$. On introduit d'abord quelques notations. Pour tous sous-groupes paraboliques relativement standards $\widetilde{Q} \subseteq \widetilde{S}$ posons :

$$
\left(\overline{\mathfrak{n}}_{\widetilde{Q}}^{\widetilde{S}}\right)^{\prime}=\overline{\mathfrak{n}}_{\widetilde{Q}}^{\widetilde{S}} \backslash \bigcup_{\widetilde{Q} \subseteq \widetilde{R} \subsetneq \widetilde{S}} \overline{\mathfrak{n}}_{\widetilde{Q}}^{\widetilde{R}}, \quad \mathfrak{m}_{\widetilde{S}, \widetilde{Q}}^{\prime}=\left(\overline{\mathfrak{n}}_{\widetilde{Q}}^{\widetilde{S}}\right)^{\prime} \oplus \mathfrak{m}_{\widetilde{Q}} \oplus \mathfrak{n}_{\widetilde{Q}}^{\widetilde{S}} .
$$

On a alors les décompositions suivantes :

$$
\overline{\mathfrak{n}} \widetilde{\widetilde{S}}=\coprod_{Q \subseteq R \subseteq S}\left(\overline{\mathfrak{n}}_{\widetilde{Q}}^{\widetilde{R}}\right)^{\prime}
$$

et $\mathfrak{m}_{\widetilde{P}}=\coprod_{\widetilde{P}} \subseteq \widetilde{S} \subseteq \widetilde{P}\left(\mathfrak{m}_{\widetilde{S}, \widetilde{1}}^{\prime} \oplus \mathfrak{n}_{\widetilde{S}}^{\widetilde{P}}\right)$ pour tout $\widetilde{P}_{1} \subseteq \widetilde{P} \subseteq \widetilde{P}_{2}$.

Finalement, en utilisant le corollaire 2.5 on obtient, pour tout $\mathfrak{o} \in \mathcal{O}$ et tout $\widetilde{P}_{1} \subseteq \widetilde{P} \subseteq \widetilde{P}_{2}$

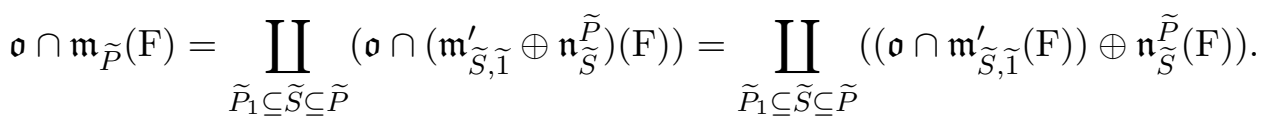

Grâce à cela, on peut réécrire $k_{\widetilde{P}, \mathfrak{o}}(x)$ comme :

$$
\sum_{\widetilde{P} 1 \subseteq \widetilde{S} \subseteq \widetilde{P}} \sum_{\eta \in \mathfrak{n} \widetilde{\widetilde{P}}(\mathrm{~F})} \sum_{\zeta \in \mathfrak{m}_{\widetilde{S}, \tilde{1}}^{\prime}(\mathrm{F}) \cap \mathfrak{0}} \int_{\mathfrak{n}_{\widetilde{P}}(\mathbb{A})} f\left(x^{-1}\left(\eta+\zeta+U_{\widetilde{P}}\right) x\right) d U_{\widetilde{P}}
$$

Fixons un caractère additif non-trivial $\psi$ sur $\mathrm{F} \backslash \mathbb{A}$. En appliquant la formule sommatoire de Poisson pour la somme portant $\operatorname{sur} \eta \in \mathfrak{n} \underset{\widetilde{S}}{\widetilde{P}}(\mathrm{~F})$ de la fonction :

$$
\mathfrak{n}_{\widetilde{S}}^{\widetilde{P}}(\mathbb{A}) \ni Y \longmapsto \int_{\mathfrak{n}_{\widetilde{P}}(\mathbb{A})} f\left(x^{-1}\left(Y+\zeta+U_{\widetilde{P}}\right) x\right) d U_{\widetilde{P}},
$$

pour tout $\zeta \in \mathfrak{m}_{\widetilde{S}, \widetilde{1}}^{\prime}(\mathrm{F}) \cap \mathfrak{o}$, on obtient

$$
k_{P, \mathfrak{o}}(x)=\sum_{\widetilde{P}_{1} \subseteq \widetilde{S} \subseteq \widetilde{P}} \sum_{\eta \in \tilde{\mathfrak{n}}_{\widetilde{S}}(\mathrm{~F})} \sum_{\zeta \in \mathfrak{m}_{\widetilde{S}, \widetilde{1}}^{\prime}(\mathrm{F}) \cap \mathfrak{o}} \Phi_{\widetilde{S}}(x, \zeta, \eta),
$$

où :

$$
\Phi_{\widetilde{S}}(x, X, Y)=\int_{\mathfrak{n}_{\widetilde{S}}(\mathbb{A})} f\left(x^{-1}\left(X+U_{\widetilde{S}}\right) x\right) \psi\left(\left\langle Y, U_{\widetilde{S}}\right\rangle\right) d U_{\widetilde{S}}, x \in G(\mathbb{A}), X \in \mathfrak{m}_{\widetilde{S}}(\mathbb{A}), Y \in \overline{\mathfrak{n}}_{\widetilde{S}}^{\widetilde{2}}(\mathbb{A}) .
$$

En utilisant l'égalité (2.8) on peut écrire $k_{\widetilde{P}, \mathfrak{o}}(x)$ aussi comme :

$$
\sum_{\widetilde{P} 1 \subseteq \widetilde{S} \subseteq \widetilde{R} \subseteq \widetilde{P}} \sum_{\zeta \in \mathfrak{m}_{\widetilde{S}, \widetilde{1}}^{\prime}(\mathrm{F}) \cap \mathfrak{a}} \sum_{\eta \in(\overline{\mathfrak{n}} \widetilde{S})^{\prime}(\mathrm{F})} \Phi_{\widetilde{S}}(x, \zeta, \eta) .
$$

Grâce à cette formule, on a pour tout $\mathfrak{o} \in \mathcal{O}$ :

$$
\begin{aligned}
& k_{\widetilde{1}, \widetilde{2}, \mathfrak{o}}(x)=\sum_{\widetilde{P}_{1} \subseteq \widetilde{P} \subseteq \widetilde{P}_{2}}(-1)^{d d_{\widetilde{P}}^{\widetilde{G}}} k_{\widetilde{P}, \mathfrak{o}}(x)= \\
& \sum_{\widetilde{P} \subseteq \widetilde{S} \subseteq \widetilde{R} \subseteq \widetilde{P} \subseteq \widetilde{P}_{2}}(-1)^{d \widetilde{\mathcal{P}}} \sum_{\zeta \in \mathfrak{m}_{\widetilde{S}, \widetilde{1}}^{\prime}(\mathrm{F}) \cap \mathfrak{o}} \sum_{\eta \in(\overline{\mathfrak{n}} \widetilde{\mathcal{S}})^{\prime}(\mathrm{F})} \Phi_{\widetilde{S}}(x, \zeta, \eta)= \\
& \sum_{\widetilde{P} 1 \subseteq \widetilde{S} \subseteq \widetilde{R} \subseteq \widetilde{P}_{2}} \sum_{\zeta \in \mathfrak{m}_{\widetilde{S}, \widetilde{1}}^{\prime}(\mathrm{F}) \cap \mathfrak{o}} \sum_{\eta \in(\overline{\mathfrak{n}} \widetilde{\widetilde{S}})^{\prime}(\mathrm{F})} \Phi_{\widetilde{S}}(x, \zeta, \eta) \sum_{\widetilde{R} \subseteq \widetilde{P} \subseteq \widetilde{P}_{2}}(-1)^{d \widetilde{\mathcal{P}}} .
\end{aligned}
$$


On invoque maintenant l'identité due à Arthur [Art78], proposition 1.1 :

$$
\sum_{\left\{\widetilde{P} \mid \widetilde{R} \subseteq \widetilde{P} \subseteq \widetilde{P}_{2}\right\}}(-1)^{d_{\widetilde{P}}^{\widetilde{P}_{2}}}=\left\{\begin{array}{l}
0 \text { si } \widetilde{R} \neq \widetilde{P}_{2}, \\
1 \text { sinon. }
\end{array}\right.
$$

On en déduit que la somme (2.9) décrivant $k_{\widetilde{1}, \widetilde{2}, \mathfrak{o}}(x)$ se réduit à :

$$
(-1)^{d \widetilde{P}_{\widetilde{P}_{2}}^{\widetilde{G}}} \sum_{\widetilde{P}_{1} \subseteq \widetilde{S} \subseteq \widetilde{P}_{2}} \sum_{\eta \in\left(\widetilde{\mathfrak{n}}_{\widetilde{S}}^{\widetilde{T}}\right)^{\prime}(\mathrm{F})} \sum_{\zeta \in \mathfrak{m}_{\widetilde{S}, \widetilde{1}}^{\prime}(\mathrm{F}) \cap \mathfrak{o}} \Phi_{\widetilde{S}}(x, \zeta, \eta) .
$$

Ainsi, pour démontrer le théorème 2.7 il suffit de majorer :

$$
\int_{P_{1}(\mathrm{~F}) \backslash G(\mathbb{A})} \chi_{\widetilde{1}, \widetilde{2}}^{T}(x) \sum_{\eta \in\left(\widetilde{\mathfrak{n}}_{\widetilde{S}}^{2}\right)^{\prime}(\mathrm{F})} \sum_{\zeta \in \mathfrak{m}_{\widetilde{S}, \widetilde{1}}^{\prime}(\mathrm{F})}\left|\Phi_{\widetilde{S}}(x, \zeta, \eta)\right| e^{\sigma \operatorname{det}\left(H_{G}(x)\right)} d x
$$

où $\widetilde{P}_{1} \subseteq \widetilde{S} \subseteq \widetilde{P}_{2}$ sont fixés. Remarquons que la double somme sur $\mathfrak{o} \in \mathcal{O}$ et $\zeta \in \mathfrak{m}_{\widetilde{S}, \widetilde{1}}^{\prime}(\mathrm{F}) \cap \mathfrak{o}$ s'est réduit à la somme sur tout $\zeta \in \mathfrak{m}_{\widetilde{S}, \widetilde{1}}^{\prime}(\mathrm{F})$.

En utilisant la décomposition d'Iwasawa $G(\mathbb{A})=P_{1}(\mathbb{A}) K$, ainsi que la décomposition $P_{1}=$ $N_{1} M_{1}$ on a que (2.11) égale

$$
\int_{K} \int_{\left[M_{1}\right]\left[N_{1}\right]} F^{\widetilde{1}}\left(m_{1}, T\right) \sigma_{\widetilde{1}}^{\widetilde{2}}\left(H_{\widetilde{1}}\left(m_{1}\right)-T_{\widetilde{1}}\right) e^{\left(\sigma \operatorname{det}-2 \rho_{1}\right)\left(H_{1}\left(m_{1}\right)\right)} \sum_{\eta \in\left(\tilde{\mathfrak{n}}_{\widetilde{S}}\right)^{\prime}(\mathrm{F})} \sum_{\zeta \in \mathfrak{m}_{\widetilde{S}, \widetilde{1}}^{\prime}(\mathrm{F})}\left|\Phi_{\widetilde{S}}\left(n_{1} m_{1} k, \zeta, \eta\right)\right| d n_{1} d m_{1} d k .
$$

Pour $\widetilde{B} \in \mathcal{P}\left(M_{\widetilde{0}}, B, \widetilde{P}\right)$ (voir définition (1.13) $)$ soit $\lambda_{\widetilde{B}, \widetilde{1}, \sigma}$ l'élément de $\left(\mathfrak{a}_{\widetilde{B}}^{\widetilde{G}}\right)^{*}$ associé à $\rho=$ $\sigma \operatorname{det}-2 \rho_{1} \in \mathfrak{a}_{1}^{*}$ par le lemme 1.6. En vertu de ce lemme il suffit de borner pour un tel $\widetilde{B} \subseteq \widetilde{P}_{1}$ fixé l'expression suivante :

$$
\int_{A_{\widetilde{B}}^{\widetilde{G}, \infty}\left(P_{\widetilde{1}}, c_{0}, T_{\widetilde{B}}\right)} \sigma_{\left[N_{1}\right]}^{\widetilde{\widetilde{1}}}\left(H_{\widetilde{1}}(a)-T_{\widetilde{1}}\right) e^{\lambda_{\widetilde{B}, \widetilde{1}, \sigma}\left(H_{\widetilde{B}}(a)\right)} \sup _{k_{1} \in \omega_{\widetilde{1}}, k \in \widetilde{K}} \sum_{\eta \in\left(\widetilde{\mathfrak{n}}_{\widetilde{S}}^{\widetilde{2}}\right)^{\prime}(\mathrm{F})} \sum_{\zeta \in \mathfrak{m}_{\widetilde{S}, \widetilde{1}}^{\prime}(\mathrm{F})}\left|\Phi_{\widetilde{S}}\left(n_{1} k_{1} a k, \zeta, \eta\right)\right| d n_{1} d a
$$

où $\omega_{\widetilde{1}}=\omega_{\widetilde{B}} \cap M_{\widetilde{1}}(\mathbb{A})$.

L'intégrale ci-dessus est essentiellement identique à celle qui apparaît dans la preuve de la proposition 4.4 dans Cha02 qui dit justement qu'une telle expression vérifie les conditions du théorème 2.7. Plus précisément, on voit qu'elle apparaît quand on passe de l'expression (4.8) à (4.9) dans loc. cit. On remarque que dans la preuve dans loc. cit. on a $\lambda_{\widetilde{B}, \widetilde{1}, \sigma}=2 \rho_{\widetilde{B}}-2 \rho_{\widetilde{P}_{1}}$, mais en fait la preuve marche sans changement pour n'importe quel $\lambda \in\left(\mathfrak{a}_{\widetilde{B}}^{\widetilde{G}}\right)^{*}$ ce qui démontre les théorèmes 2.7 et 2.6.

\section{Propriétés qualitatives}

On fixe une fois pour toute $\eta: \mathrm{F} \backslash \mathbb{A}^{*} \rightarrow \mathbb{C}^{*}$ un caractère continu, qui est trivial sur le groupe $\mathbb{R}_{>0}^{*}$ vu comme un sous-groupe de $\mathbb{A}^{*}$ via l'inclusion $\mathbb{R}_{>0}^{*} \hookrightarrow(\mathrm{F} \otimes \mathbb{Q} \mathbb{R})^{*} \hookrightarrow \mathbb{A}^{*}$. Notons alors pour $s \in \mathbb{C}$ et $x \in \mathbb{A}^{*}:$

$$
\eta_{s}(x)=|x|_{\mathbb{A}}^{s} \eta(x) .
$$

Pour une fonction $f \in \mathcal{S}(\widetilde{\mathfrak{g}}(\mathbb{A}))$ et $T \in T_{+}+\mathfrak{a}_{\tilde{0}}^{+}$on note

$$
k^{T}(x)=k_{f}^{T}(x)=\sum_{\mathfrak{o} \in \mathcal{O}} k_{f, \mathfrak{o}}^{T}(x), \quad x \in G(\mathrm{~F}) \backslash G(\mathbb{A}) .
$$


Grâce au théorème 2.6 les distributions suivantes :

$$
\begin{array}{ll}
I_{\mathfrak{o}}^{T}\left(\eta_{s}, f\right)=\int_{G(\mathrm{~F}) \backslash G(\mathbb{A})} k_{f, \mathfrak{o}}^{T}(x) \eta_{s}(\operatorname{det} x) d x, & \mathfrak{o} \in \mathcal{O}, T \in T_{+}+\mathfrak{a}_{\tilde{0}}^{+}, \\
I^{T}\left(\eta_{s}, f\right) & =\int_{G(\mathrm{~F}) \backslash G(\mathbb{A})} k_{f}^{T}(x) \eta_{s}(\operatorname{det} x) d x
\end{array}
$$

sont bien définies pour tout $s \in \mathbb{C}$.

Dans le paragraphe 3.3 on démontrera que pour $s \in \mathbb{C}$ la fonction $T \mapsto I_{\mathfrak{o}}^{T}\left(\eta_{s}, f\right)$ est un polynôme-exponentielle et si $s \neq-1,1$ son terme purement polynomial, noté $I_{\mathfrak{o}}\left(\eta_{s}, f\right)$, ne dépend pas de $T$. Pour bien énoncer ce résultat on étudie d'abord les fonctions de type polynômeexponentielle dans le paragraphe 3.1 et dans le paragraphe 3.2 on introduit les distributions $I_{\mathfrak{o}}^{M_{\widetilde{Q}}, T}\left(\eta_{s}, \cdot\right)$ pour tout sous-groupe parabolique relativement standard $\widetilde{Q}$ de $\widetilde{G}$. La suite de cette section est consacrée aux propriétés des distributions $I_{\mathfrak{o}}\left(\eta_{s}, \cdot\right)$ pour $s \neq-1,1$.

\subsection{Polynômes-exponentielles}

Soit $\mathcal{V}$ un $\mathbb{R}$-espace vectoriel de dimension finie. Par un polynôme-exponentielle sur $\mathcal{V}$ on entend une fonction sur $\mathcal{V}$ de la forme

$$
f(v)=\sum_{\lambda \in \mathcal{V}^{*}} e^{\lambda(v)} P_{\lambda}(v), \quad v \in \mathcal{V}
$$

où $P_{\lambda}$ est un polynôme sur $\mathcal{V}$ à coefficients complexes, égale 0 pour presque tout $\lambda \in \mathcal{V}^{*}$. On appelle $\lambda \in \mathcal{V}^{*}$ tels que $P_{\lambda} \neq 0$ les exposants de $f$ et le polynôme correspondant à $\lambda=0$ le terme purement polynomial de $f$. On a alors le résultat d'unicité suivant : si $f$ est comme ci-dessus et $g=\sum_{\lambda \in \mathcal{V}^{*}} e^{\lambda} Q_{\lambda}$ est un polynôme-exponentielle sur $\mathcal{V}$ tel que $g(v)=f(v)$ pour tout $v \in \mathcal{V}$ alors pour tout $\lambda \in \mathcal{V}^{*}$ on a $P_{\lambda}=Q_{\lambda}$.

Pour $i=0,1, \ldots, n$ soit $e_{i}^{*} \in \mathfrak{a}_{\tilde{0}}^{*}$ le caractère par lequel $A_{\widetilde{0}}$ agit sur $D_{i}$. Posons aussi $e_{j}^{\vee} \in \mathfrak{a}_{\tilde{0}}$ les éléments tels que $e_{i}^{*}\left(e_{j}^{\vee}\right)=\delta_{i j}$ où $i, j=0,1, \ldots, n$. On pose pour $i=1, \ldots, n$

$\widetilde{\varpi}_{i}^{-}=\frac{n+1-i}{n+1}\left(\sum_{j=1}^{i} e_{j}^{*}\right)-\frac{i}{n+1}\left(e_{0}^{*}+\sum_{j=i+1}^{n} e_{i}^{*}\right), \quad \widetilde{\varpi}_{i}^{+}=\frac{n+1-i}{n+1}\left(e_{0}^{*}+\sum_{j=1}^{i-1} e_{j}^{*}\right)-\frac{i}{n+1}\left(\sum_{j=i}^{n} e_{i}^{*}\right)$.

On définit $\widetilde{\varpi}_{i}^{-, \vee}, \widetilde{\varpi}_{i}^{+, \vee}$ en remplaçant ${ }^{*}$ par $\vee$. Alors $\widetilde{\varpi}_{i}^{-}, \widetilde{\varpi}_{i}^{+} \in\left(\mathfrak{a}_{\tilde{0}}^{\widetilde{G}}\right)^{*}$. On pose aussi $\widetilde{\varpi}_{l}^{-}=$ $\widetilde{\varpi}_{l}^{+}=0$ pour $l \notin\{1, \ldots, n\}$.

Fixons un sous-groupe parabolique relativement standard $\widetilde{Q}$ de $\widetilde{G}$ stabilisant le drapeau

$$
0=V_{i_{0}} \subsetneq \cdots \subsetneq V_{i_{k-1}} \subsetneq V_{i_{k}} \oplus D_{0} \subsetneq \cdots \subsetneq V_{i_{l}} \oplus D_{0}=W
$$

Alors

$$
\begin{aligned}
& \widehat{\Delta}_{\widetilde{Q}}=\left\{\widetilde{\varpi}_{i_{a}}^{-}, \widetilde{\varpi}_{i_{b}+1}^{+} \mid 1 \leq a \leq k-1, k \leq b \leq l-1\right\}, \\
& \widehat{\Delta}_{\widetilde{Q}}^{\vee}=\left\{\widetilde{\varpi}_{i_{a}}^{-, \vee \vee}, \widetilde{\varpi}_{i_{b}+1}^{+, \vee} \mid 1 \leq a \leq k-1, k \leq b \leq l-1\right\} .
\end{aligned}
$$

Posons $\widetilde{\varpi}_{\widetilde{Q}}^{-}:=\widetilde{\varpi}_{m}^{-}$où $m=\max \left(\left\{j \mid \widetilde{\varpi}_{j}^{-} \in \widehat{\Delta}_{\widetilde{Q}}\right\} \cup\{0\}\right)$. De même, on pose $\widetilde{\varpi}_{\widetilde{Q}}^{+}:=\widetilde{\varpi}_{m}^{+}$où $m=\min \left(\left\{j \mid \widetilde{\varpi}_{j}^{+} \in \widehat{\Delta}_{\widetilde{Q}}\right\} \cup\{0\}\right)$. Pour $s \in \mathbb{C}$ posons :

$$
s_{\widetilde{Q}}:=\frac{s(n+1)+i_{k-1}+i_{k}-n}{i_{k}-i_{k-1}+1}
$$

et

$$
\rho_{\widetilde{Q}, s}:=\left(1+s_{\widetilde{Q}}\right) \widetilde{\varpi} \widetilde{Q}+\left(1-s_{\widetilde{Q}}\right) \widetilde{\varpi} \widetilde{Q}_{\widetilde{Q}}^{+} \in\left(\mathfrak{a}_{\widetilde{Q}, \mathbb{C}}^{\widetilde{G}}\right)^{*} .
$$

Avec la notation du paragraphe 1.7 on a : 
Lemme 3.1. Soient $s \in \mathbb{C}$ et $\widetilde{Q} \in \mathcal{F}\left(M_{\widetilde{0}}, B\right)$. Alors

1.

$$
\rho_{\widetilde{Q}, s}=\iota_{\widetilde{Q}}^{s t}\left(s \operatorname{det}-2 \rho_{Q}\right)+2 \rho_{\widetilde{Q}}
$$

où l'on voit $s \operatorname{det}-2 \rho_{Q}$ comme l'élément de $\left(\mathfrak{a}_{\widetilde{Q}, \mathbb{C}}^{s t}\right)^{*}$ par restriction.

2. Pour tout $m \in H_{\widetilde{Q}}(\mathbb{A})^{1} \times G_{\widetilde{Q}}(\mathbb{A})$ on a

$$
e^{-\rho_{\widetilde{Q}, s}\left(H_{\widetilde{Q}}(m)\right)}|\operatorname{det} m|_{\mathbb{A}}^{s}=|\operatorname{det} m|_{\mathbb{Q}}^{s \widetilde{Q}} .
$$

3. Pour tout $\widetilde{R} \in \mathcal{F}\left(M_{\widetilde{0}}, B\right)$ tel que $\widetilde{R} \supseteq \widetilde{Q}$, la restriction de $\rho_{\widetilde{Q}, s}$ à $\widetilde{R}$ égale $\rho_{\widetilde{R}, s}$. Démonstration. Calcul direct.

Lemme 3.2. Soit $\widetilde{Q} \in \mathcal{F}\left(M_{\widetilde{0}}, B\right)$.

i) Pour tout $s \in \mathbb{C} \backslash\{-1,1\}$ et tout $\widetilde{\varpi}^{\vee} \in \widehat{\Delta}_{\widetilde{Q}}^{\vee}$ on a $\rho_{\widetilde{Q}, s}\left(\widetilde{\varpi}^{\vee}\right) \neq 0$.

ii) Pour tout $s \in \mathbb{C}$ tel que $-1<\operatorname{Re}(s)<1$ et tout $\widetilde{\varpi}^{\vee} \in \widehat{\Delta}_{\widetilde{Q}}^{\vee}$ on a $\operatorname{Re}\left(\rho_{\widetilde{Q}, s}\left(\widetilde{\varpi}^{\vee}\right)\right)>0$.

Démonstration. On a pour $1 \leq a \leq k-1$ que $\rho_{\widetilde{Q}_{, s}}\left(\widetilde{\varpi}_{i_{a}}^{-, \vee}\right)=i_{a}(1+s)$ et pour $k \leq b \leq l-1$ que $\rho_{\widetilde{Q}, s}\left(\widetilde{\varpi}_{i_{b}+1}^{+, \vee}\right)=\left(n-i_{b}\right)(1-s)$, d'où les résultats voulus.

On note le corollaire immédiat du lemme 3.2 .

Corollaire 3.3. Soit $\widetilde{Q}$ un sous-groupe parabolique relativement standard de $\widetilde{G}$. Alors, pour tout sous-groupe parabolique $\widetilde{R} \supseteq \widetilde{Q}$ différent de $\widetilde{G}$ et tout $s \in \mathbb{C} \backslash\{-1,1\}$ la restriction de $\rho_{\widetilde{Q}, s} \grave{a} \mathfrak{a}_{\widetilde{R}}^{\widetilde{G}}$ est non-nulle.

Soit $v_{\widetilde{Q}}$ le volume dans $\mathfrak{a}_{\widetilde{Q}}^{\widetilde{G}}$ du parallélotope engendré par $\left(\widehat{\Delta}_{\widetilde{Q}}\right)^{\vee}$. Suivant le paragraphe 2 de Art81, posons

$$
\hat{\theta}_{\widetilde{Q}}(\mu)=v_{\widetilde{Q}}^{-1} \prod_{\widetilde{\varpi} \in \widehat{\Delta}_{\widetilde{Q}}^{\vee}} \mu\left(\widetilde{\varpi}^{\vee}\right), \quad \mu \in \mathfrak{a}_{\widetilde{Q}, \mathbb{C}}^{*} \cdot
$$

Supposons $\widetilde{R} \supseteq \widetilde{Q}$, pour $X \in \mathfrak{a}_{\widetilde{Q}}$ on note $X_{\widetilde{R}}$ sa projection à $\mathfrak{a}_{\widetilde{R}}$ selon la décomposition (1.2). Suivant loc. cit., posons

$$
\Gamma_{\widetilde{Q}}^{\prime}(H, X)=\sum_{\widetilde{R} \supseteq \widetilde{Q}}(-1)^{d d_{\widetilde{Q}}^{\widetilde{Q}}} \hat{\tau}_{\widetilde{R}}\left(H_{\widetilde{R}}-X_{\widetilde{R}}\right) \tau_{\widetilde{Q}}^{\widetilde{R}}(H), \quad H, X \in \mathfrak{a}_{\widetilde{Q}} .
$$

Lemme 3.4. Soit $\widetilde{Q} \in \mathcal{F}\left(M_{\widetilde{0}}, B\right)$. Alors, pour tout $s \in \mathbb{C}$

$$
p_{\widetilde{Q}, s}(X):=\int_{\mathfrak{a}_{\widetilde{Q}}^{s t}} e^{\left(s \operatorname{det}+2 \rho_{\widetilde{Q}}-2 \rho_{Q}\right)(H)} \Gamma_{\widetilde{Q}}^{\prime}(H, X) d H, \quad X \in \mathfrak{a}_{\widetilde{Q}}
$$

est un polynôme-exponentielle sur $\mathfrak{a}_{\widetilde{Q}} / \mathfrak{a}_{\widetilde{G}}$. En plus, si $s \neq-1,1$, pour tout $\widetilde{R} \supseteq \widetilde{Q}$ il existe un polynôme $P_{\widetilde{Q}, \widetilde{R}, s}$ de degré au plus $d_{\widetilde{Q}}^{\widetilde{G}}$ sur $\mathfrak{a}_{\widetilde{R}} / \mathfrak{a}_{\widetilde{G}}$ tel que

$$
p_{\widetilde{Q}, s}(X):=j_{\widetilde{Q}}^{-1} \sum_{\widetilde{R} \supseteq \widetilde{Q}} e^{\rho_{\widetilde{R}, s}\left(X_{\widetilde{R}}\right)} p_{\widetilde{Q}, \widetilde{R}, s}\left(X_{\widetilde{R}}\right)
$$

ò̀ $p_{\widetilde{Q}, \widetilde{G}, s}\left(X_{\widetilde{G}}\right)=(-1)^{d{ }^{\widetilde{G}}} \hat{\theta}_{\widetilde{Q}}\left(\rho_{\widetilde{Q}, s}\right)^{-1}$. En particulier, si $s \neq-1,1$, la fonction $p_{\widetilde{Q}, s}$ est un

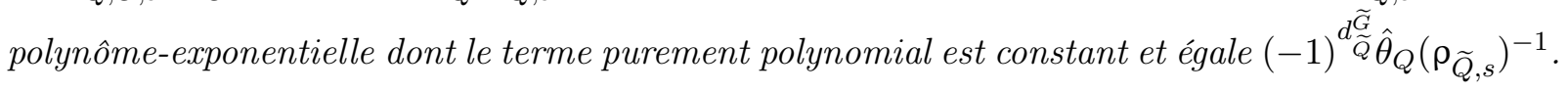


Remarque 3.5. On ne prétend pas que les polynômes $p_{\widetilde{Q}, \widetilde{R}, s}$ sont uniquement déterminés pour tout $\widetilde{R} \supseteq \widetilde{Q}$. En effet, il arrive que $\rho_{\widetilde{R}, s}=\rho_{\widetilde{R}^{\prime}, s}$ pour $\widetilde{R} \neq \widetilde{R}^{\prime}$. Cependant, $\widetilde{G}$ est le seul sous-groupe parabolique $\widetilde{R} \supseteq \widetilde{Q}$ tel que $\rho_{\widetilde{R}, s}=0$ si $s \neq-1,1$ d'où l'unicité du terme $p_{\widetilde{Q}, \widetilde{G}, s}$.

Démonstration. En utilisant le lemme 3.1 et l'équation (1.11) on a

$$
p_{\widetilde{Q}, s}(X)=j_{\widetilde{Q}}^{-1} \int_{\mathfrak{a} \widetilde{G}} e^{\rho_{\widetilde{Q}, s}(H)} \Gamma_{\widetilde{Q}}^{\prime}(H, X) d H
$$

Il résulte du lemme 2.1 de [Art81] que pour un $X$ fixé, la fonction $\mathfrak{a}_{\widetilde{Q}}^{\widetilde{G}} \ni H \mapsto \Gamma_{\widetilde{Q}}^{\prime}(H, X)$ est une fonction caractéristique d'un compact dans $\mathfrak{a}_{\widetilde{Q}}^{\widetilde{G}}$. L'intégrale ci-dessus est donc bien définie et le lemme 2.2 dans loc. cit. montre que c'est un polynôme-exponentielle ce qui démontre la première partie du lemme.

Quand $s \neq-1,1$, on applique le lemme $3.2 i$ ) et son corollaire 3.3 et on voit qu'on est dans la même situation que dans le lemme 3.3 de [Zyd15a]. En utilisant ce lemme on conclut.

\subsection{Une généralisation du théorème 2.6}

Soient $W^{\prime}$ un F-espace vectoriel de dimension $m+1, V^{\prime} \subseteq W^{\prime}$ un sous-espace de dimension $m$ et $D_{0}^{\prime} \subseteq\left(W^{\prime} \backslash V^{\prime}\right) \cup\{0\}$ une droite, où $m \in \mathbb{N}$. Soient $H \cong \prod_{i=1}^{k} \mathrm{GL}_{n_{i}} G^{\prime}=\mathrm{GL}\left(V^{\prime}\right)$ et $\widetilde{G}^{\prime}=\mathrm{GL}\left(W^{\prime}\right)$ où $k \in \mathbb{N}$, et $n_{i} \in \mathbb{N}$ pour $i=1, \ldots, k$. On identifie $G^{\prime}$ avec le sous-groupe de $\widetilde{G}^{\prime}$ qui agit trivialement sur $D_{0}^{\prime}$. On va généraliser le théorème 2.6] au cas de l'inclusion $H \times G^{\prime} \hookrightarrow H \times \widetilde{G}^{\prime}$.

Notons $\mathfrak{h}=\operatorname{Lie}(H), \mathfrak{g}^{\prime}=\operatorname{Lie}\left(G^{\prime}\right)$ et $\widetilde{\mathfrak{g}}^{\prime}=\operatorname{Lie}\left(\widetilde{G}^{\prime}\right)$. Pour $X \in\left(\mathfrak{h} \times \widetilde{\mathfrak{g}}^{\prime}\right)(\mathrm{F})$ soient $X_{1} \in \mathfrak{h}(\mathrm{F})$ et $X_{2} \in \widetilde{\mathfrak{g}}^{\prime}(\mathrm{F})$ tels que $X=X_{1}+X_{2}$. Soit $\mathcal{O}^{H \times \widetilde{G}^{\prime}}$ la relation d'équivalence sur $\left(\mathfrak{h} \times \widetilde{\mathfrak{g}}^{\prime}\right)(\mathrm{F})$ définie de la façon suivante. On a $X=X_{1}+X_{2} \sim Y=Y_{1}+Y_{2}$ si et seulement si les polynômes caractéristiques de $X_{1}$ et $Y_{1}$ coïncident et si $X_{2}$ et $Y_{2}$ sont dans la même classe pour la relation d'équivalence dans $\widetilde{\mathfrak{g}}^{\prime}(\mathrm{F})$ décrite dans le paragraphe 2.1 par rapport à l'inclusion $G^{\prime} \hookrightarrow \widetilde{G}^{\prime}$.

Soit $B$ un sous-F-groupe de Borel de $H \times G^{\prime}$ et fixons aussi $M_{0}$ une partie de Levi de $B$. Soit $M_{\widetilde{0}}$ l'unique sous-groupe de Levi minimal de $H \times \widetilde{G}^{\prime}$ tel que $M_{\widetilde{0}} \supseteq M_{0}$. On peut alors parler de sous-groupes paraboliques standards de $H \times G^{\prime}$ et semi-standards de $H \times \widetilde{G}^{\prime}$. Notons $\mathcal{F}_{H \times G^{\prime}}\left(M_{\widetilde{0}}, B\right)$ le sous-ensemble de sous-groupes paraboliques semi-standards $\widetilde{P}$ de $H \times \widetilde{G}^{\prime}$ tels que $\widetilde{P} \supseteq B$.

Fixons un sous-groupe de Borel $\widetilde{B}_{0} \in \mathcal{F}\left(M_{\widetilde{0}}\right)$. Soit $\widetilde{P} \in \mathcal{F}\left(M_{\widetilde{0}}\right)$. Pour tout $H \in \mathfrak{a}_{\widetilde{B}_{0}}$ on note $H_{\widetilde{P}}$ la projection de $s H$ à $\mathfrak{a}_{\widetilde{P}}$ où $s$ est un élément du groupe de Weyl de $H \times \widetilde{G}^{\prime}$ tel que $s^{-1} \widetilde{P} \supseteq \widetilde{B}_{0}$.

Pour une fonction $f \in \mathcal{S}\left(\left(\mathfrak{h} \times \widetilde{\mathfrak{g}}^{\prime}\right)(\mathbb{A})\right)$, un $\widetilde{P} \in \mathcal{F}_{H \times G^{\prime}}\left(M_{\widetilde{0}}, B\right)$ et une classe $\mathfrak{o} \in \mathcal{O}^{H \times \widetilde{G}^{\prime}}$ on pose

$$
k_{f, \widetilde{P}, \mathfrak{o}}(x)=\sum_{\xi \in \mathfrak{m}_{\widetilde{P}}(\mathrm{~F}) \cap \mathfrak{o}} \int_{\mathfrak{n}_{\widetilde{P}}(\mathbb{A})} f\left(x^{-1}\left(\xi+U_{\widetilde{P}}\right) x\right) d U_{\widetilde{P}}, \quad x \in\left(H \times \widetilde{G}^{\prime}\right)(\mathbb{A}) .
$$

Pour un $T \in \mathfrak{a}_{\widetilde{B}_{0}}^{+}$on pose donc

$$
k_{f, \mathfrak{o}}^{T}(x)=\sum_{\widetilde{P} \in \mathcal{F}_{H \times G^{\prime}}\left(M_{\widetilde{0}}, B\right)}(-1)^{d_{\widetilde{P}}^{H \times \widetilde{G}^{\prime}}} \sum_{\delta \in P(\mathrm{~F}) \backslash\left(H \times G^{\prime}\right)(\mathrm{F})} \hat{\tau}_{\widetilde{P}}^{H \times \widetilde{G}^{\prime}}\left(H_{\widetilde{P}}(\delta x)-T_{\widetilde{P}}\right) k_{f, \widetilde{P}, \mathfrak{o}}(\delta x)
$$

où $P=\widetilde{P} \cap\left(H \times G^{\prime}\right)$. 
Théoréme 3.6. Soit $f \in \mathcal{S}\left(\left(\mathfrak{h} \times \widetilde{\mathfrak{g}}^{\prime}\right)(\mathbb{A})\right)$, alors pour tout $T \in \mathfrak{a}_{\widetilde{B}_{0}}^{+}$suffisamment régulier et tout $\sigma \in \mathbb{R}$ on a

$$
\sum_{\mathfrak{o} \in \mathcal{O}^{H \times \widetilde{G}^{\prime}}} \int_{\left(H \times G^{\prime}\right)(\mathrm{F}) \backslash H(\mathbb{A})^{1} \times G^{\prime}(\mathbb{A})}\left|k_{f, \mathfrak{o}}^{T}(x)\right||\operatorname{det} x|_{\mathbb{A}}^{\sigma} d x<\infty .
$$

Démonstration. La preuve est similaire à celle du théorème 2.6 Les détails sont laissés au lecteur. Notons que si $f=f_{1} \otimes f_{2}$ où $f \in \mathcal{S}(\mathfrak{h}(\mathbb{A}))$ et $f_{2} \in \mathcal{S}\left(\widetilde{\mathfrak{g}}^{\prime}(\mathbb{A})\right)$, c'est une conséquence immédiate des théorèmes 2.6 ci-dessus et 3.1 de [Cha02].

Notons alors pour $s \in \mathbb{C}, \mathfrak{o} \in \mathcal{O}^{H \times \widetilde{G}^{\prime}}$ et $f \in \mathcal{S}\left(\left(\mathfrak{h} \times \widetilde{\mathfrak{g}}^{\prime}\right)(\mathbb{A})\right)$

$$
I_{\mathfrak{o}}^{H \times \widetilde{G}^{\prime}, T}\left(\eta_{s}, f\right)=\int_{\left(H \times G^{\prime}\right)(\mathrm{F}) \backslash H(\mathbb{A})^{1} \times G^{\prime}(\mathbb{A})} k_{f, \mathfrak{o}}^{T}(x) \eta_{s}(\operatorname{det} x) d x .
$$

Revenons dans le contexte de l'inclusion $G \hookrightarrow \widetilde{G}^{\prime}$. Soit $\widetilde{Q}$ un sous-groupe parabolique relativement standard de $\widetilde{G}$. Comme il est expliqué dans le paragraphe 1.6, on a les décompositions $M_{\widetilde{Q}} \cong H_{\widetilde{Q}} \times \widetilde{G}_{\widetilde{Q}}$ et $M_{Q} \cong H_{\widetilde{Q}} \times G_{\widetilde{Q}}$ où $H_{\widetilde{Q}}, G_{\widetilde{Q}}$ et $\widetilde{G}_{\widetilde{Q}}$ vérifient les conditions de ce paragraphe.

Soit $\mathfrak{o} \in \mathcal{O}$, il existe $\mathfrak{o}_{\widetilde{Q}, 1}, \ldots, \mathfrak{o}_{\widetilde{Q}, m} \in \mathcal{O}^{M_{\widetilde{Q}}}$, où $0 \leq m<\infty$, tels que

$$
\mathfrak{m}_{\widetilde{Q}}(\mathrm{~F}) \cap \mathfrak{o}=\coprod_{i=1}^{m} \mathfrak{o}_{\widetilde{Q}, i} \cap \mathfrak{m}_{\widetilde{Q}}(\mathrm{~F}) .
$$

Pour $T \in \mathfrak{a}_{\tilde{0}}^{+}$et $s \in \mathbb{C}$, on définit alors les distributions $I_{\mathfrak{o}}^{M_{\widetilde{Q}}, T}\left(\eta_{s}, \cdot\right)$ et $I^{M_{\widetilde{Q}}, T}\left(\eta_{s}, \cdot\right) \operatorname{sur} \mathcal{S}\left(\mathfrak{m}_{\widetilde{Q}}(\mathbb{A})\right)$ par :

$$
I_{\mathfrak{o}}^{M_{\widetilde{Q}}, T}\left(\eta_{s}, f\right)=\sum_{i=1}^{m} I_{\mathfrak{o}_{\widetilde{Q}, i}}^{M_{\widetilde{Q}}, T}\left(\eta_{s_{\widetilde{Q}}}, f\right), \quad I^{M_{\widetilde{Q}}, T}\left(\eta_{s}, f\right)=\sum_{\mathfrak{o} \in \mathcal{O}} I_{\mathfrak{o}}^{M_{\widetilde{Q}}, T}\left(\eta_{s}, f\right)
$$

où $s_{\widetilde{Q}}$ est défini par (3.1) et pour $\mathfrak{o}_{\widetilde{Q}} \in \mathcal{O}^{M_{\widetilde{Q}}}, I_{\mathfrak{o}_{\widetilde{Q}}}^{M_{\widetilde{Q}}, T}\left(\eta_{s}, \cdot\right)$ c'est la distribution associée à l'inclusion $M_{Q} \hookrightarrow M_{\widetilde{Q}}$ décrite ci-dessus par rapport au sous-groupe de Levi minimal $M_{0}$ de $M_{Q}$ et aux sous-groupes de Borel $B \cap M_{Q}$ de $M_{Q}$ et $\widetilde{B}_{0} \cap M_{\widetilde{Q}}$ de $M_{\widetilde{Q}}$.

Pour $f \in \mathcal{S}(\widetilde{\mathfrak{g}}(\mathbb{A}))$ on pose

$$
f_{\widetilde{Q}}(X)=\int_{K} \int_{\mathfrak{n}_{\widetilde{Q}}(\mathbb{A})} f\left(k^{-1}\left(X+U_{\widetilde{Q}}\right) k\right) \eta(\operatorname{det} k) d U_{\widetilde{Q}} d k, \quad X \in \mathfrak{m}_{\widetilde{Q}}(\mathbb{A}) ;
$$

alors $f_{\widetilde{Q}} \in \mathcal{S}\left(\mathfrak{m}_{\widetilde{Q}}(\mathbb{A})\right)$. Notons que l'application

$$
\widetilde{Q} \supseteq \widetilde{P} \mapsto M_{\widetilde{Q}} \cap \widetilde{P}
$$

définit une bijection entre les sous-groupes paraboliques relativement standards de $\widetilde{G}$ contenus dans $\widetilde{Q}$ et les sous-groupes paraboliques semi-standards de $M_{\widetilde{Q}}$ contenant $B \cap M_{Q}$. En utilisant le lemme 3.1, on s'aperçoit alors que pour tout sous-groupe de Borel relativement standard $\widetilde{B} \subseteq \widetilde{Q}$ et tous $T \in \mathfrak{a}_{\tilde{0}}^{+}$et $s \in \mathbb{C}$ on a

$$
\begin{aligned}
& I_{\mathfrak{o}}^{M_{\widetilde{Q}}, T}\left(\eta_{s}, f_{\widetilde{Q}}\right)=\int_{M_{Q}(\mathrm{~F}) \backslash H_{Q}(\mathbb{A})^{1} \times G_{\widetilde{Q}}(\mathbb{A})^{j=1}} \sum_{k_{\widetilde{Q}}, \mathfrak{Q}_{\widetilde{Q}, i}}^{T_{\widetilde{Q}}}(m) \eta_{s_{\widetilde{Q}}}(\operatorname{det} m) d m=\int_{M_{Q}(\mathrm{~F}) \backslash H_{Q}(\mathbb{A})^{1} \times G_{\widetilde{Q}}(\mathbb{A})} e^{-\rho_{\widetilde{Q}, s}\left(H_{\widetilde{Q}}(m)\right)} \sum_{\widetilde{P} \subseteq \widetilde{Q}}(-1)^{d \widetilde{P}} \\
& \sum_{\eta \in\left(P \cap M_{Q}\right)(\mathrm{F}) \backslash M_{Q}(\mathrm{~F})} \hat{\tau}_{\widetilde{Q}}^{\widetilde{Q}}\left(H_{\widetilde{P}}(\eta m)-T_{\widetilde{P}}\right)\left(\sum_{\xi \in \mathfrak{m}_{\widetilde{P}}(\mathrm{~F}) \cap \mathfrak{0}} \int_{\mathfrak{n}_{\widetilde{P}}(\mathbb{A})} f_{\widetilde{Q}}\left(\operatorname{Ad}\left((\eta m)^{-1}\right)\left(\xi+U_{\widetilde{P}}^{\widetilde{Q}}\right)\right) d U_{\widetilde{P}}^{\widetilde{Q}}\right) \eta_{s}(\operatorname{det} m) d m .
\end{aligned}
$$




\subsection{Le comportement en $T$}

On démontre la proposition suivante.

Proposition 3.7. Soient $f \in \mathcal{S}(\widetilde{\mathfrak{g}}(\mathbb{A})), T^{\prime} \in T_{+}+\mathfrak{a}_{\tilde{0}}^{+}, s \in \mathbb{C}, \mathfrak{o} \in \mathcal{O}$ et $T \in T^{\prime}+\mathfrak{a}_{\tilde{0}}^{+}$. Alors

$$
I_{\mathfrak{o}}^{T}\left(\eta_{s}, f\right)=\sum_{\widetilde{Q} \in \mathcal{F}\left(M_{\widetilde{0}}, B\right)} p_{\widetilde{Q}, s}\left(T_{\widetilde{Q}}-T_{\widetilde{Q}}^{\prime}\right) e^{\rho_{\widetilde{Q}, s}\left(T_{\widetilde{Q}}^{\prime}\right)} I_{\mathfrak{o}}^{M_{\widetilde{Q}}, T^{\prime}}\left(\eta_{s}, f_{\widetilde{Q}}\right)
$$

où pour un sous-groupe parabolique $\widetilde{Q}$ relativement standard, la fonction $p_{\widetilde{Q}, s}$ est définie dans le lemme 3.4, $\rho_{\widetilde{Q}, s} \in\left(\mathfrak{a}_{\widetilde{Q}}^{\widetilde{G}}\right)^{*}$ est défini par [3.2, la distribution $I_{\mathfrak{o}}^{M_{\widetilde{Q}}, T^{\prime}}$ est définie dans le paragraphe 3.2 et $f_{\widetilde{Q}} \in \mathcal{S}\left(\mathfrak{m}_{\widetilde{Q}}(\mathbb{A})\right)$ est définie par (3.7) dans le même paragraphe.

Démonstration. Il est démontré dans le le paragraphe 2 de [Art81, que les fonctions $\Gamma_{\widetilde{Q}}^{\prime}$, définies par (3.4), vérifient la relation suivante : pour tout sous-groupe parabolique relativement standard $\widetilde{P}$ de $\widetilde{G}$, on a :

$$
\hat{\tau}_{\widetilde{P}}(H-X)=\sum_{\widetilde{Q} \supseteq \widetilde{P}}(-1)^{d d_{\widetilde{Q}}^{\widetilde{G}}} \hat{\tau}_{\widetilde{P}}^{\widetilde{Q}}(H) \Gamma_{\widetilde{Q}}^{\prime}(H, X), \quad H, X \in \mathfrak{a}_{\widetilde{P}}
$$

Fixons un $T^{\prime} \in T_{+}+\mathfrak{a}_{\tilde{0}}^{+}$et soit $T \in T^{\prime}+\mathfrak{a}_{\tilde{0}}^{+}$. En utilisant l'égalité ci-dessus dans la définition du noyau $k_{\mathfrak{o}}^{T}(2.3)$ avec $H=H_{\widetilde{P}}(\delta x)-T_{\widetilde{P}}^{\prime}$ et $X=T_{\widetilde{P}}-T_{\widetilde{P}}^{\prime}$ pour tout $\widetilde{P} \in \mathcal{F}\left(M_{\widetilde{0}}, B\right)$ et tout $\delta \in P(\mathrm{~F}) \backslash G(\mathrm{~F})$ on a

$$
\begin{array}{r}
I_{\mathfrak{o}}^{T}\left(\eta_{s}, f\right)=\int_{G(\mathrm{~F}) \backslash G(\mathbb{A})} \sum_{\widetilde{P} \in \mathcal{F}\left(M_{\widetilde{0}}, B\right)}(-1)^{d \widetilde{\widetilde{P}}_{\widetilde{P}}^{\widetilde{T}}} \sum_{\delta \in P(\mathrm{~F}) \backslash G(\mathrm{~F})} \sum_{\widetilde{Q} \supseteq \widetilde{P}}(-1)^{d \widetilde{\widetilde{Q}}} \Psi_{\widetilde{P}, \widetilde{Q}_{\mathfrak{o}}}^{T, T^{\prime}}(\delta x) \eta_{s}(\operatorname{det} x) d x= \\
\sum_{\widetilde{Q} \in \mathcal{F}\left(M_{\widetilde{0}}, B\right)_{Q(\mathrm{~F}) \backslash G(\mathbb{A})}} \sum_{\mathcal{F}\left(M_{\widetilde{0}}, B\right) \ni \widetilde{P} \subseteq \widetilde{Q}}(-1)^{d \widetilde{P}} \sum_{\delta \in\left(P \cap M_{Q}\right)(\mathrm{F}) \backslash M_{Q}(\mathrm{~F})} \Psi_{\widetilde{P}, \widetilde{Q}, \mathfrak{o}}^{T, T^{\prime}}(\delta x) \eta_{s}(\operatorname{det} x) d x
\end{array}
$$

où :

$$
\Psi_{\widetilde{P}, \widetilde{Q}, \mathfrak{o}}^{T, T^{\prime}}(x)=k_{\widetilde{P}, \mathfrak{o}}(x) \hat{\tau}_{\widetilde{P}}^{\widetilde{Q}}\left(H_{\widetilde{P}}(x)-T_{\widetilde{P}}^{\prime}\right) \Gamma_{\widetilde{Q}}^{\prime}\left(H_{\widetilde{P}}(x)-T_{\widetilde{Q}}^{\prime}, T_{\widetilde{Q}}-T_{\widetilde{Q}}^{\prime}\right) .
$$

Le fait qu'on peut sortir la somme $\sum_{\widetilde{Q}}$ avant l'intégrale va se déduire du fait qu'on va montrer que les intégrales correspondantes sont absolument convergentes.

Fixons $\widetilde{Q} \in \mathcal{F}\left(M_{\widetilde{0}}, B\right)$. On remplace l'intégrale sur $Q(\mathrm{~F}) \backslash G(\mathbb{A})$ par l'intégrale sur

$$
N_{Q}(\mathrm{~F}) \backslash N_{Q}(\mathbb{A}) \times A_{\widetilde{Q}}^{s t, \infty} \times\left(M_{Q}(\mathrm{~F}) \backslash H_{\widetilde{Q}}(\mathbb{A})^{1} \times G_{\widetilde{Q}}(\mathbb{A})\right) \times K
$$

ce qui donne $d x=e^{-2 \rho_{Q}\left(H_{Q}(a m)\right)} d n d a d m d k$.

Soient $m \in\left(H_{\widetilde{Q}}(\mathbb{A})^{1} \times G_{\widetilde{Q}}(\mathbb{A})\right), a \in A_{\widetilde{Q}}^{s t, \infty}, k \in K, \delta \in M_{Q}(\mathrm{~F})$ et $\widetilde{P} \subseteq \widetilde{Q}$. On a donc :

$$
\int_{N_{Q}(\mathrm{~F}) \backslash N_{Q}(\mathbb{A})} \Psi_{\widetilde{P}, \widetilde{Q}, \mathfrak{o}}^{T, T^{\prime}}(\delta n a m k) d n=\int_{N_{Q}(\mathrm{~F}) \backslash N_{Q}(\mathbb{A})} \Psi_{P, Q, \mathfrak{o}}^{T, T^{\prime}}(n a \delta m k) d n
$$

car $\delta \in M_{Q}(\mathrm{~F})$ normalise $N_{Q}(\mathbb{A})$ sans changer sa mesure et il commute avec $A_{\widetilde{Q}}^{s t, \infty} \subseteq A_{Q}^{\infty}$. Les

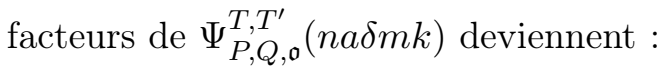

$$
\begin{gathered}
\Gamma_{\widetilde{Q}}^{\prime}\left(H_{\widetilde{Q}}(n a \delta m k)-T_{\widetilde{Q}}^{\prime}, T_{\widetilde{Q}}-T_{\widetilde{Q}}^{\prime}\right)=\Gamma_{\widetilde{Q}}^{\prime}\left(H_{\widetilde{Q}}(a)+H_{\widetilde{Q}}(m)-T_{\widetilde{Q}}^{\prime}, T_{\widetilde{Q}}-T_{\widetilde{Q}}^{\prime}\right), \\
\hat{\tau}_{\widetilde{P}}^{\widetilde{Q}}\left(H_{\widetilde{P}}(n a \delta m k)-T_{\widetilde{P}}^{\prime}\right)=\hat{\tau}_{\widetilde{P}}^{\widetilde{Q}}\left(H_{\widetilde{P}}(\delta m)+H_{\widetilde{P}}(a)-T_{\widetilde{P}}^{\prime}\right)=\hat{\tau}_{\widetilde{P}}^{\widetilde{Q}}\left(H_{\widetilde{P}}(\delta m)-T_{\widetilde{P}}^{\prime}\right) .
\end{gathered}
$$


Quant à $k_{\widetilde{P}, \mathfrak{o}}(n a \eta m k)$, on fait le changement de variable $\left(a^{-1} n^{-1}\left(\xi+U_{\widetilde{P}}\right) n a-\xi\right) \mapsto U_{\widetilde{P}}$ (voir la définition de $k_{\widetilde{P}, \mathfrak{o}}$ au début du paragraphe 2.2) et l'on obtient :

$$
k_{\widetilde{P}, \mathfrak{o}}(n a \delta m k)=e^{2 \rho_{\widetilde{Q}}\left(H_{\widetilde{Q}}(a)\right)} \int_{\mathfrak{n}_{\widetilde{P}}(\mathbb{A})} \sum_{\xi \in \mathfrak{m}_{\widetilde{P}}(\mathrm{~F}) \cap \mathfrak{o}} f\left((\delta m k)^{-1}\left(\xi+U_{\widetilde{P}}\right) \delta m k\right) d U_{\widetilde{P}}
$$

Ensuite, comme la mesure de $N_{Q}(\mathrm{~F}) \backslash N_{Q}(\mathbb{A})$ vaut 1 on a en faisant le changement de variable $\left((\delta m)^{-1}\left(\xi+U_{\widetilde{Q}}\right) \delta m-\xi\right) \mapsto U_{\widetilde{Q}}$

$$
\begin{array}{r}
e^{-2 \rho_{\widetilde{Q}}\left(H_{\widetilde{Q}}(a)\right)} \int_{K} \int_{\left[N_{Q}\right]} k_{\widetilde{P}, \mathfrak{o}}(n a \delta m k) \eta(\operatorname{det} k) d n d k=\int_{K} \int_{\mathfrak{n}_{\widetilde{P}}(\mathbb{A})} \sum_{\xi \in \mathfrak{m}_{\widetilde{P}}(\mathrm{~F}) \cap \mathfrak{o}} f\left((\delta m k)^{-1}\left(\xi+U_{\widetilde{P}}\right) \delta m k\right) \eta(\operatorname{det} k) d U_{\widetilde{P}} d k \\
=\int_{\mathfrak{n} \widetilde{Q}(\mathbb{A})} \int_{K} \int_{\mathfrak{n}_{\widetilde{Q}}(\mathbb{A})} \sum_{\xi \in \mathfrak{m}_{\widetilde{P}}(\mathrm{~F}) \cap \mathfrak{o}} f\left((\delta m k)^{-1}\left(\xi+U_{\widetilde{P}}^{\widetilde{Q}}+U_{\widetilde{Q}}\right) \delta m k\right) \eta(\operatorname{det} k) d U_{\widetilde{Q}} d k d U_{\widetilde{P}}^{\widetilde{Q}}= \\
=e^{2 \rho_{\widetilde{Q}}\left(H_{\widetilde{Q}}(m)\right)} \int_{\mathfrak{n}_{\widetilde{P}}(\mathbb{A})} \sum_{\xi \in \mathfrak{m}_{\widetilde{P}}(\mathrm{~F}) \cap \mathfrak{o}} f_{\widetilde{Q}}\left((\delta m)^{-1}\left(\xi+U_{\widetilde{P}}^{\widetilde{Q}}\right) \delta m\right) d U_{\widetilde{P}}^{\widetilde{Q}}
\end{array}
$$

où $f_{\widetilde{Q}} \in \mathcal{S}\left(\mathfrak{m}_{\widetilde{Q}}(\mathbb{A})\right)$ est définie par (3.7) dans le paragraphe 3.2

Remarquons qu'on a $2 \rho_{\widetilde{Q}}\left(H_{\widetilde{Q}}(m)\right)=2 \rho_{Q}\left(H_{Q}(m)\right)$. En utilisant les lemmes 3.1 et 3.4 ainsi que l'égalité (1.11) on voit donc que l'intégrale sur $A_{\widetilde{Q}}^{s t, \infty}$ se réduit alors à

$$
\int_{A_{\widetilde{Q}}^{s t, \infty}} \Gamma_{\widetilde{Q}}^{\prime}\left(H_{\widetilde{Q}}(a)+H_{\widetilde{Q}}(m)-T_{\widetilde{Q}}^{\prime}, T_{\widetilde{Q}}-T_{\widetilde{Q}}^{\prime}\right) e^{\left(s \operatorname{det}+2\left(\rho_{\widetilde{Q}}-\rho_{Q}\right)\right)\left(H_{\widetilde{Q}}(a)\right)} d a=e^{\rho_{\widetilde{Q}, s}\left(T_{\widetilde{Q}}^{\prime}-H_{\widetilde{Q}}(m)\right)} p_{\widetilde{Q}, s}\left(T_{\widetilde{Q}}-T_{\widetilde{Q}}^{\prime}\right) .
$$

En utilisant le calcul (3.11) et en regardant la relation (3.8), on s'aperçoit qu'avec la notation de l'équation (3.10) on a

$\int_{Q(\mathrm{~F}) \backslash G(\mathbb{A})} \sum_{\widetilde{P} \subseteq \widetilde{Q}}(-1)^{d d_{\widetilde{P}}^{\widetilde{Q}}} \sum_{\delta \in\left(P \cap M_{Q}\right)(\mathrm{F}) \backslash M_{Q}(\mathrm{~F})} \Psi_{\widetilde{P}, \widetilde{Q}, \mathfrak{o}}^{T, T^{\prime}}(\delta x) \eta_{s}(\operatorname{det} x) d x=e^{\rho_{\widetilde{Q}, s}\left(T_{\widetilde{Q}}^{\prime}\right)} p_{\widetilde{Q}, s}\left(T_{\widetilde{Q}}-T_{\widetilde{Q}}^{\prime}\right) I_{\mathfrak{o}}^{M_{\widetilde{Q}}, T^{\prime}}\left(\eta_{s}, f_{\widetilde{Q}}\right)$.

Ce qu'il fallait démontrer.

En utilisant la proposition 3.7 démontrée ci-dessus et le lemme 3.4 qui décrit les fonctions $p_{\widetilde{Q}}$ explicitement on obtient le comportement en $T$ des distributions $I_{\mathfrak{o}}^{T}$ et $I^{T}$.

Théoréme 3.8. Soit $f \in \mathcal{S}(\widetilde{\mathfrak{g}}(\mathbb{A}))$. Les fonctions $T \mapsto I_{\mathfrak{o}}^{T}\left(\eta_{s}, f\right)$ et $T \mapsto I^{T}\left(\eta_{s}, f\right)$ où $\mathfrak{o} \in \mathcal{O}$, $s \in \mathbb{C}$ et $T$ parcourt $T_{+}+\mathfrak{a}_{\tilde{0}}^{+}$sont des polynômes-exponentielles. De plus, si $s \neq-1,1$ leur parties purement polynomiales sont constantes et données respectivement par

$$
\begin{aligned}
I_{\mathfrak{o}}\left(\eta_{s}, f\right) & :=\sum_{\widetilde{Q} \in \mathcal{F}\left(M_{\widetilde{0}}, B\right)}(-1)^{d d_{\widetilde{Q}}^{\widetilde{G}}} j_{\widetilde{Q}}^{-1} \hat{\theta}_{\widetilde{Q}}\left(\rho_{\widetilde{Q}, s}\right)^{-1} e^{\rho_{\widetilde{Q}, s}\left(T_{\widetilde{Q}}^{\prime}\right)} I_{\mathfrak{o}}^{M_{\widetilde{Q}}, T^{\prime}}\left(\eta_{s}, f_{\widetilde{Q}}\right), \\
I\left(\eta_{s}, f\right) & :=\sum_{\widetilde{Q} \in \mathcal{F}\left(M_{\widetilde{0}}, B\right)}(-1)^{d{ }^{\widetilde{G}}} j_{\widetilde{Q}}^{-1} \hat{\theta}_{\widetilde{Q}}\left(\rho_{\widetilde{Q}, s}\right)^{-1} e^{\rho_{\widetilde{Q}, s}\left(T_{\widetilde{Q}}^{\prime}\right)} I^{M_{\widetilde{Q}}, T^{\prime}}\left(\eta_{s}, f_{\widetilde{Q}}\right),
\end{aligned}
$$

pour tout $T^{\prime} \in T_{+}+\mathfrak{a}_{\tilde{0}}^{+}$. En particulier, les distributions $I_{\mathfrak{o}}$ et $I$ ne dépendent pas de $T^{\prime}$.

Remarque 3.9. Soit $\widetilde{Q}$ un sous-groupe parabolique relativement standard de $\widetilde{G}$. Par le même raisonnement que dans la proposition 3.7 on obtient que pour tout $s \in \mathbb{C}$ les distributions $I_{\mathfrak{o}}^{M_{\widetilde{Q}}, T}\left(\eta_{s}, \cdot\right)$ et $I^{M_{\widetilde{Q}}, T}\left(\eta_{s}, \cdot\right)$, définies dans le paragraphe 3.2. sont des polynômes-exponentielles en $T$ qui ne dépendent pas de $T_{\widetilde{Q}} \in \mathfrak{a}_{\widetilde{Q}}$. Cependant, si $\widetilde{Q} \neq \widetilde{G}$ le terme purement polynomial n'est pas constant. 
Remarque 3.10. En vertu de la convergence absolue, pour tout $\widetilde{Q} \in \mathcal{F}\left(M_{\widetilde{0}}, B\right)$ et tout $f \in$ $\mathcal{S}\left(\mathfrak{m}_{\widetilde{Q}}(\mathbb{A})\right)$ on a que les fonctions $\mathbb{C} \ni s \mapsto I^{M_{\widetilde{Q}}, T}\left(\eta_{s}, f\right)$ et $\mathbb{C} \ni s \mapsto I_{\mathfrak{o}}^{M_{\widetilde{Q}}, T}\left(\eta_{s}, f\right)$, où o $\in \mathcal{O}$, sont holomorphes. Le théorème 3.8 dit alors que pour tout $f \in \mathcal{S}(\widetilde{\mathfrak{g}}(\mathbb{A}))$ et tout $\mathfrak{o} \in \mathcal{O}$ les fonctions $\mathbb{C} \backslash\{-1,1\} \ni s \mapsto I^{T}\left(\eta_{s}, f\right)$ et $\mathbb{C} \backslash\{-1,1\} \ni s \mapsto I_{\mathfrak{o}}^{T}\left(\eta_{s}, f\right)$ sont holomorphes et admettent des prolongement méromorphes à $\mathbb{C}$ avec des pôles possibles en -1 et 1 .

\section{4 Équivariance}

Soient $f \in \mathcal{S}(\widetilde{\mathfrak{g}}(\mathbb{A}))$ et $y \in G(\mathbb{A})$. Notons $f^{y} \in \mathcal{S}(\widetilde{\mathfrak{g}}(\mathbb{A}))$ la fonction définie par $f^{y}(X)=$ $f(\operatorname{Ad}(y) X)$.

On voit que $I_{\mathfrak{o}}^{T}\left(\eta_{s}, f^{y}\right)$ pour $T \in T_{+}+\mathfrak{a}_{\tilde{0}}^{+}$et $s \in \mathbb{C}$ égale

$$
\int_{G(\mathrm{~F}) \backslash G(\mathbb{A})} \sum_{\widetilde{P} \in \mathcal{F}\left(M_{\widetilde{0}}, B\right)}(-1)^{d d_{\widetilde{P}}^{\widetilde{\widetilde{P}}}} \sum_{\delta \in P(\mathrm{~F}) \backslash G(\mathrm{~F})} k_{\widetilde{P}, \mathfrak{o}}(\delta x) \hat{\tau}_{\widetilde{P}}\left(H_{\widetilde{P}}(\delta x y)-T_{\widetilde{P}}\right) \eta_{s}(\operatorname{det}(x y)) d x .
$$

Pour $x \in G(\mathbb{A})$ et $P \in \mathcal{F}(B)$ soit $k_{P}(x)$ un élément de $K$ tel que $x k_{P}(x)^{-1} \in P(\mathbb{A})$. Alors, en utilisant l'égalité (3.9) on a :

$$
\hat{\tau}_{\widetilde{P}}\left(H_{\widetilde{P}}(\delta x y)-T_{\widetilde{P}}\right)=\sum_{\widetilde{Q} \supseteq \widetilde{P}}(-1)^{d{ }^{\widetilde{G}}} \hat{\tau}_{\widetilde{P}}^{\widetilde{Q}}\left(H_{\widetilde{P}}(\delta x)-T_{\widetilde{P}}\right) \Gamma_{\widetilde{Q}}^{\prime}\left(H_{\widetilde{P}}(\delta x)-T_{\widetilde{P}},-H_{\widetilde{P}}\left(k_{P}(\delta x) y\right)\right)
$$

d'où on obtient que $I_{\mathfrak{o}}^{T}\left(\eta_{s}, f^{y}\right)$ égale la somme sur $\widetilde{Q} \in \mathcal{F}\left(M_{\widetilde{0}}, B\right)$ de

$$
\int_{Q(\mathrm{~F}) \backslash G(\mathbb{A})} \Gamma_{\widetilde{Q}}^{\prime}\left(H_{\widetilde{Q}}(x)-T_{\widetilde{Q}},-H_{\widetilde{Q}}\left(k_{Q}(x) y\right)\right) \sum_{\widetilde{P} \subseteq \widetilde{Q}}(-1)^{d d_{\widetilde{P}}^{\widetilde{Q}}} \sum_{\delta \in P(\mathrm{~F}) \cap M_{Q}(\mathrm{~F}) \backslash M_{Q(\mathrm{~F})}} k_{\widetilde{P}}(\delta x) \hat{\tau}_{\widetilde{Q}}\left(H_{\widetilde{P}}(\delta x)-T_{\widetilde{P}}\right) \eta_{s}(\operatorname{det}(x y)) d x .
$$

Soit $x=n a m k$ où $n \in N_{Q}(\mathrm{~F}) \backslash N_{Q}(\mathbb{A}), m \in M_{Q}(\mathrm{~F}) \backslash\left(H_{\widetilde{Q}}(\mathbb{A})^{1} \times G_{\widetilde{Q}}(\mathbb{A})\right), a \in A_{\widetilde{Q}}^{s t, \infty}$ et $k \in K$. Donc $d x=e^{-2 \rho_{Q}\left(H_{Q}(a m)\right)} d n d a d m d k$ et pour $\delta \in M_{Q}(\mathrm{~F})$ on a

$$
\Gamma_{\widetilde{Q}}^{\prime}\left(H_{\widetilde{Q}}(\delta n a m k)-T_{\widetilde{Q}},-H_{\widetilde{Q}}\left(k_{Q}(\delta n a m k) y\right)\right)=\Gamma_{\widetilde{Q}}^{\prime}\left(H_{\widetilde{Q}}(a)+H_{\widetilde{Q}}(m)-T_{\widetilde{Q}},-H_{\widetilde{Q}}(k y)\right) .
$$

Ensuite, en faisant les mêmes opérations comme dans (3.11) au début de la preuve de la proposition 3.7 on s'aperçoit qu'on a pour $\widetilde{P} \subseteq \widetilde{Q}, m \in M_{Q}(\mathrm{~F}) \backslash\left(H_{\widetilde{Q}}(\mathbb{A})^{1} \times G_{\widetilde{Q}}(\mathbb{A})\right)$ et $\delta \in M_{Q}(\mathrm{~F})$

$$
\begin{gathered}
\iint_{A_{\widetilde{Q}}^{s t, \infty}} \int_{\left.N_{Q}\right]} e^{\left(s \operatorname{det}-2 \rho_{Q}\right)\left(H_{Q}(a m)\right)} k_{\widetilde{P}, \mathfrak{o}}(\delta n m a k) \Gamma_{\widetilde{Q}}^{\prime}\left(H_{\widetilde{P}}(\delta n a m k)-T_{\widetilde{Q}},-H_{\widetilde{P}}\left(k_{P}(\delta n a m k) y\right)\right) \eta(\operatorname{det} k) \\
d n d k d a=|\operatorname{det} m|_{\mathbb{A}}^{s \widetilde{Q}} e^{\rho_{\widetilde{Q}, s}\left(T_{\widetilde{Q}}\right)} \int_{\mathfrak{n}_{\widetilde{P}}(\mathbb{A})^{\xi}} \sum_{\xi \in \mathfrak{m}_{\widetilde{P}}(\mathrm{~F}) \cap \mathfrak{o}} \int_{K} \int_{\mathfrak{a}_{\widetilde{\widetilde{Q}}}} \int_{\mathfrak{n}_{\widetilde{Q}}} e^{\rho_{\widetilde{Q}, s}(H)} f\left(k^{-1}\left((m \delta)^{-1}\left(\xi+U_{\widetilde{P}}^{\widetilde{Q}}\right) \delta m+U_{\widetilde{Q}}\right) k\right) \eta(\operatorname{det} k) \\
\Gamma_{\widetilde{Q}}^{\prime}\left(H,-H_{\widetilde{Q}}(k y)\right) d U_{\widetilde{Q}} d H d k d U_{\widetilde{P}}^{\widetilde{Q}}=|\operatorname{det} m|_{\mathbb{A}}^{s \widetilde{Q}} e^{\rho_{\widetilde{Q}, s}\left(T_{\widetilde{Q}}\right)} \int_{\mathfrak{n}_{\widetilde{Q}}(\mathbb{A})^{\xi \in \mathfrak{m}_{\widetilde{P}}(\mathrm{~F}) \cap \mathfrak{o}}} f_{\widetilde{Q}, s, y}\left((m \delta)^{-1}\left(\xi+U_{\widetilde{P}}^{\widetilde{Q}}\right) m \delta\right) d U_{\widetilde{P}}^{\widetilde{Q}}
\end{gathered}
$$

où l'on pose

$$
f_{\widetilde{Q}, s, y}(X)=\int_{K} \int_{\mathfrak{n}_{\widetilde{Q}}(\mathbb{A})} f\left(k^{-1}\left(X+U_{\widetilde{Q}}\right) k\right) u_{\widetilde{Q}, s}^{\prime}(k, y) \eta(\operatorname{det} k) d U_{\widetilde{Q}} d k, \quad X \in \mathfrak{m}_{\widetilde{Q}}(\mathbb{A})
$$

où

$$
u_{\widetilde{Q}, s}^{\prime}(k, y)=\int_{\mathfrak{a}_{\widetilde{Q}}^{\widetilde{G}}} e^{\rho_{\widetilde{Q}, s}(H)} \Gamma_{\widetilde{Q}}^{\prime}\left(H,-H_{\widetilde{Q}}(k y)\right) d H, \quad k \in K, s \in \mathbb{C} .
$$


La fonction $K \ni k \mapsto u_{\widetilde{Q}, s}^{\prime}(k, y)$ étant continue on a bien $f_{\widetilde{Q}, s, y} \in \mathcal{S}\left(\mathfrak{m}_{\widetilde{Q}}(\mathbb{A})\right)$. On obtient le théorème suivant.

Théoréme 3.11. Soient $y \in G(\mathbb{A}), s \in \mathbb{C}$ et $f \in \mathcal{S}(\widetilde{\mathfrak{g}}(\mathbb{A}))$. Les distributions $I_{\mathfrak{o}}^{T}\left(\eta_{s}, \cdot\right)$ vérifient

$$
I_{\mathfrak{o}}^{T}\left(\eta_{s}, f^{y}\right)-\eta_{s}(\operatorname{det} y) I_{\mathfrak{o}}^{T}\left(\eta_{s}, f\right)=\eta_{s}(\operatorname{det} y) \sum_{\widetilde{Q} \in \mathcal{F}\left(M_{\widetilde{0}}, B\right) \backslash\{\widetilde{G}\}} e^{\rho_{\widetilde{Q}, s}\left(T_{\widetilde{Q}}\right)} I_{\mathfrak{o}}^{M_{\widetilde{Q}}, T}\left(\eta_{s}, f_{\widetilde{Q}, s, y}\right)
$$

où les distributions $I_{\mathfrak{o}}^{M_{\widetilde{Q}}, T}\left(\eta_{s}, \cdot\right)$ sur $\mathcal{S}\left(\mathfrak{m}_{\widetilde{Q}}(\mathbb{A})\right)$ sont définies par (3.6). En particulier, pour $s \neq$ $-1,1$ on a

$$
I_{\mathfrak{o}}\left(\eta_{s}, f^{y}\right)=\eta_{s}(\operatorname{det} y) I_{\mathfrak{o}}\left(\eta_{s}, f\right), \quad I\left(\eta_{s}, f^{y}\right)=\eta_{s}(\operatorname{det} y) I\left(\eta_{s}, f\right) .
$$

Démonstration. La formule pour la différence $I_{\mathfrak{o}}^{T}\left(\eta_{s}, f^{y}\right)-\eta_{s}(\operatorname{det} y) I_{\mathfrak{o}}^{T}\left(\eta_{s}, f\right)$ est claire après les calculs qu'on a faits. Si $s \neq-1,1$, cette formule-ci démontre aussi l' $\eta_{s}$-invariance de la distribution $I_{\mathfrak{o}}\left(\eta_{s}, \cdot\right)$, car si $\widetilde{Q} \subsetneq \widetilde{G}$, d'après la remarque 3.9, le terme $I_{\mathfrak{o}}^{M_{\widetilde{Q}}, T}\left(\eta_{s}, f_{\widetilde{Q}, y, s}\right)$ est un polynôme-exponentielle en $T$ qui ne dépend pas de $T_{\widetilde{Q}}$. En outre $\rho_{\widetilde{Q}, s}$ est non-trivial sur $\mathfrak{a}_{\widetilde{Q}}^{\widetilde{G}}$ en vertu du lemme 3.2. Il en découle que $e^{\rho_{\widetilde{Q}, s}\left(T_{\widetilde{Q}}\right)} I_{\mathfrak{o}}^{M_{\widetilde{Q}}, T}\left(\eta_{s}, f_{\widetilde{Q}, y, s}\right)$ n'a pas de terme constant dans ce cas et par conséquent les termes constants de $I_{\mathfrak{o}}^{T}\left(\eta_{s}, f^{y}\right)$ et $\operatorname{de} \eta_{s}(\operatorname{det} y) I_{\mathfrak{o}}^{T}\left(\eta_{s}, f\right)$ coïncident.

\subsection{Indépendance des choix}

Soit $s \in \mathbb{C} \backslash\{-1,1\}$ et $\mathfrak{o} \in \mathcal{O}$. Dans ce paragraphe on démontrera que la distribution $I_{\mathfrak{o}}\left(\eta_{s}, \cdot\right)$ ne dépend d'aucun choix, sauf le choix d'une mesure de Haar sur $G(\mathbb{A})$ et les choix des mesures sur les sous-F-espaces $\mathcal{V}$ de $\widetilde{\mathfrak{g}}$, notre choix étant que $\mathcal{V}(\mathrm{F}) \backslash \mathcal{V}(\mathbb{A})$ soit de volume 1.

Remarquons d'abord que $I_{\mathfrak{o}}\left(\eta_{s}, \cdot\right)$ ne dépend pas du choix du sous-groupe de Borel $\widetilde{B}_{0} \in$ $\mathcal{P}\left(M_{\widetilde{0}}\right)$ choisi au début du paragraphe 1.8. En effet, ce choix intervient seulement dans le choix du chambre positive. Soit $\widetilde{B} \in \mathcal{P}\left(M_{\widetilde{0}}\right)$ et $\sigma \in \Omega^{\widetilde{G}}$ tel que $\sigma \widetilde{B}_{0}=\widetilde{B}$. Notons $I_{\widetilde{B}, \mathfrak{o}}\left(\eta_{s}, \cdot\right)$ et $I_{\widetilde{B}, \mathfrak{o}}^{T}\left(\eta_{s}, \cdot\right)$ les distributions obtenues à partir de $\widetilde{B}$. Alors si $T \in \mathfrak{a}_{\widetilde{B}}^{+}$, on a $I_{\widetilde{B}, \mathfrak{o}}^{T}\left(\eta_{s}, \cdot\right)=I_{\mathfrak{o}}^{\sigma^{-1}} T\left(\eta_{s}, \cdot\right)$ et l'indépendance suit du théorème 3.8

Démontrons que $I_{\mathfrak{o}}\left(\eta_{s}, \cdot\right)$ ne dépend pas du choix du sous-groupe de Borel $B$ de $G$ contenant $M_{0}$. Soient $B^{\prime} \in \mathcal{P}\left(M_{0}\right)$ et $\sigma \in \Omega^{G}$ tel que $B^{\prime}=\sigma^{-1} B$. Notons $\widetilde{B}^{\prime}=\sigma^{-1} \widetilde{B}_{0}$. Notons $I_{B^{\prime}, \mathfrak{o}}^{T}\left(\eta_{s}, \cdot\right)$ et $I_{B^{\prime}, \mathfrak{o}}\left(\eta_{s}, \cdot\right)$ les distributions construites par rapport au sous-groupes de Borel $B^{\prime}$ et $\widetilde{B}^{\prime}$. C'est une conséquence simple de la relation (1.9) qu'on a $I_{\mathfrak{o}}^{T}\left(\eta_{s}, \cdot\right)=I_{B^{\prime}, \mathrm{o}}^{\sigma^{-1} T}\left(\eta_{s}, \cdot\right)$. Le théorème 3.8 permet de déduire de nouveau alors que $I_{\mathfrak{o}}\left(\eta_{s}, \cdot\right)=I_{B^{\prime}, \mathfrak{o}}\left(\eta_{s}, \cdot\right)$.

Le compact maximal $\widetilde{K}$ qu'on a choisi dans le paragraphe 1.6 dépend du choix des vecteurs non-nuls dans les droites $D_{i}$ où $i=1, \ldots, n$. Tout autre compact $\widetilde{K}^{*}$ de ce type défini par rapport à $M_{\widetilde{0}}$ égale donc $\gamma K \gamma^{-1}$ pour un $\gamma \in A_{B}(\mathrm{~F})$. Si l'on note alors $I_{\widetilde{K}^{*}, \mathfrak{o}}$ la distribution défini par rapport au mêmes données que $I_{\mathfrak{o}}$ mais pour le compact $\widetilde{K}^{*}=\gamma K \gamma^{-1}$ où $\gamma \in A_{B}(\mathrm{~F})$ on voit que $I_{\mathrm{K}^{*} \mathfrak{o}}\left(\eta_{s}, f\right)=I_{\mathfrak{o}}\left(\eta_{s}, f^{\gamma}\right)$ ce qui égale $I_{\mathfrak{o}}\left(\eta_{s}, f\right)$ en vertu du théorème 3.11 et du fait que $\eta_{s}$ est trivial sur $\mathrm{F}^{*}$. La distribution $I_{\mathfrak{o}}\left(\eta_{s}, \cdot\right)$ ne dépend pas alors du choix de sous-groupe compact maximal "standard" par rapport à $M_{0}$.

Il nous reste à démontrer l'indépendance du choix de sous-groupe de Levi minimal de $G$ (car $M_{\widetilde{0}}$ est déterminé par celui-ci). Soit $M_{0}^{\prime}=y M_{0} y^{-1}$ un sous-F-groupe de Levi minimal de $G$, où $y \in G(\mathrm{~F})$. On note alors $I_{M_{0}^{\prime}, \mathfrak{o}}$ la distribution définie par rapport au sous-groupe parabolique minimal $y B y^{-1}$, le compact maximal $y \widetilde{K} y^{-1}$ de $\widetilde{G}(\mathbb{A})$ et le sous-groupe de Borel $y \widetilde{B}_{0} y^{-1}$ de $\widetilde{G}$. On trouve alors $I_{M_{0}^{\prime}, \mathfrak{o}}\left(\eta_{s}, f\right)=I_{\mathfrak{o}}\left(\eta_{s}, f^{y}\right)$ et le résultat découle du théorème 3.11 de nouveau. 


\subsection{Orbites semi-simples régulières}

Soit $\mathcal{O}_{\text {reg }} \subseteq \mathcal{O}$ l'ensemble des orbites semi-simples régulières, c'est-à-dire des orbites composées d'éléments semi-simples réguliers. La preuve de la proposition suivante est quasiment identique à la preuve de son homologue dans le paragraphe 3.6 de [Zyd15a.

Proposition 3.12. Pour tous $f \in \mathcal{S}(\widetilde{\mathfrak{g}}(\mathbb{A})), T \in T_{+}+\mathfrak{a}_{\tilde{0}}^{+}, s \in \mathbb{C}, \mathfrak{o} \in \mathcal{O}_{\text {reg }}$ et $X \in \mathfrak{o}$ on a

$$
I_{\mathfrak{o}}^{T}\left(\eta_{s}, f\right)=I_{\mathfrak{o}}\left(\eta_{s}, f\right)=\int_{G(\mathbb{A})} f\left(x^{-1} X x\right) \eta_{s}(\operatorname{det} x) d x
$$

où l'intégrale est absolument convergente.

\section{Formule des traces infinitésimale}

Il résulte de l'analyse faite dans le paragraphe 2.1 qu'on a la décomposition de $\widetilde{\mathfrak{g}}$ en sous-F-espaces stables sous l'action de $G$ suivante :

$$
\widetilde{\mathfrak{g}}=\mathfrak{t}_{1} \oplus \mathfrak{t}_{2} \oplus \mathfrak{t}_{3}
$$

où $\mathfrak{t}_{1}=\mathfrak{g}, \mathfrak{t}_{2}=V \times V^{*}$ et $\mathfrak{t}_{3}=\mathbb{G}_{a}$. Soit $\mathfrak{t} \subseteq \tilde{\mathfrak{g}}$ un sous-F-espace défini comme une somme directe de certains d'entre les $\mathfrak{t}_{i}, i=1,2,3$. Il y a donc huit possibilités pour $\mathfrak{t}$. Puisque chaque $\mathfrak{t}_{i}$ est $G$-stable et la restriction de la forme trace $\langle\cdot, \cdot\rangle$, à $\mathfrak{t}_{i}$ est non-dégénérée, il en est de même pour t. Pour $X \in \widetilde{\mathfrak{g}}(\mathbb{A})$ soit $X_{\mathfrak{t}}$ la projection de $X$ à $\mathfrak{t}(\mathbb{A})$ selon la décomposition (4.1) ci-dessus.

Fixons $\psi$ un caractère non-trivial de $\mathrm{F} \backslash \mathbb{A}$. Pour $\mathfrak{t}$ comme ci-dessus, notons $\mathcal{F}_{\mathfrak{t}}$ l'opérateur sur $\mathcal{S}(\widetilde{\mathfrak{u}}(\mathbb{A}))$ suivant

$$
\mathcal{F}_{\mathfrak{t}}(f)(X)=\int_{\mathfrak{t}(\mathbb{A})} f\left(X-X_{\mathfrak{t}}+Y_{\mathfrak{t}}\right) \psi\left(\left\langle X_{\mathfrak{t}}, Y_{\mathfrak{t}}\right\rangle\right) d Y_{\mathfrak{t}}, \quad f \in \mathcal{S}(\widetilde{\mathfrak{g}}(\mathbb{A})), \quad X \in \widetilde{\mathfrak{g}}(\mathbb{A})
$$

où $d Y_{\mathfrak{t}}$ c'est la mesure de Haar sur $\mathfrak{t}(\mathbb{A})$ pour laquelle le volume de $\mathfrak{t}(\mathrm{F}) \backslash \mathfrak{t}(\mathbb{A})$ vaut 1 .

Théoréme 4.1. Pour tout $f \in \mathcal{S}(\widetilde{\mathfrak{g}}(\mathbb{A}))$ et tout $s \in \mathbb{C} \backslash\{-1,1\}$ on a

$$
\sum_{\mathfrak{o} \in \mathcal{O}} I_{\mathfrak{o}}\left(\eta_{s}, f\right)=\sum_{\mathfrak{o} \in \mathcal{O}} I_{\mathfrak{o}}\left(\eta_{s}, \mathcal{F}_{\mathfrak{t}}(f)\right)
$$

Démonstration. Soit $T \in T_{+}+\mathfrak{a}_{0}^{+}$et $s \in \mathbb{C}$ tel que $-1<\operatorname{Re}(s)<1$. On se place dans le contexte de la preuve du théorème 2.6, En utilisant l'identité (2.6) et l'analyse qui suit l'équation (2.7) on a que $\sum_{\mathfrak{o} \in \mathcal{O}} I_{\mathfrak{o}}^{T}\left(\eta_{s}, f\right)-\sum_{\mathfrak{o} \in \mathcal{O}} I_{\mathfrak{o}}^{T}\left(\eta_{s}, \mathcal{F}_{\mathfrak{t}}(f)\right)=I^{T}\left(\eta_{s}, f\right)-I^{T}\left(\eta_{s}, \mathcal{F}_{\mathfrak{t}}(f)\right)$ vaut

$$
\begin{aligned}
\int_{[G]}\left(k_{\widetilde{G}, \widetilde{G}}(x, f)-k_{\widetilde{G}, \widetilde{G}}\left(x, \mathcal{F}_{\mathfrak{t}}(f)\right)+\sum_{\widetilde{P}_{1} \subsetneq \widetilde{P}_{2}} \sum_{\delta \in P_{1}(\mathrm{~F}) \backslash G(\mathrm{~F})}\right. \\
\\
\left.\chi_{\widetilde{P}_{1}, \widetilde{P}_{2}}^{T}(\delta x)\left(k_{\widetilde{P}_{1}, \widetilde{P}_{2}}(\delta x, f)-k_{\widetilde{P}_{1}, \widetilde{P}_{2}}\left(\delta x, \mathcal{F}_{\mathfrak{t}}(f)\right)\right)\right) \eta_{s}(\operatorname{det} x) d x
\end{aligned}
$$

où pour les sous-groupes paraboliques relativement standards $\widetilde{P}_{1} \subseteq \widetilde{P}_{2}$ et $\phi \in \mathcal{S}(\widetilde{\mathfrak{g}}(\mathbb{A}))$ on pose

$$
k_{\widetilde{P}_{1}, \widetilde{P}_{2}}(x, \phi)=\sum_{\widetilde{P}_{1} \subseteq \widetilde{P} \subseteq \widetilde{P}_{2}}(-1)^{d{ }^{\widetilde{\widetilde{P}}}}\left(\sum_{\mathfrak{o} \in \mathcal{O}} k_{\phi, \widetilde{P}, \mathfrak{o}}(x)\right), x \in P_{1}(\mathrm{~F}) \backslash G(\mathbb{A}) .
$$

On a alors pour tout $x \in G(\mathbb{A})$

$$
k_{\widetilde{G}, \widetilde{G}}(x, f)=\sum_{\xi \in \tilde{\mathfrak{g}}(\mathrm{F})} f\left(x^{-1} \xi x\right)=\sum_{\xi \in \widetilde{\mathfrak{g}}(\mathrm{F})} \mathcal{F}_{\mathfrak{t}}(f)\left(x^{-1} \xi x\right)=k_{\widetilde{G}, \widetilde{G}}\left(x, \mathcal{F}_{\mathfrak{t}}(f)\right)
$$


grâce à la formule sommatoire de Poisson. On s'aperçoit alors que $\sum_{\mathfrak{o} \in \mathcal{O}} I_{\mathfrak{o}}^{T}\left(\eta_{s}, f\right)-\sum_{\mathfrak{o} \in \mathcal{O}} I_{\mathfrak{o}}^{T}\left(\eta_{s}, \mathcal{F}_{\mathfrak{t}}(f)\right)$ c'est en fait la somme sur $\widetilde{P}_{1} \subsetneq \widetilde{P}_{2}$ de

$$
\left.\int_{P_{1}(\mathrm{~F}) \backslash G(\mathbb{A})} \chi_{\widetilde{P}_{1}, \widetilde{P}_{2}}^{T}(x)\left(k_{\widetilde{P}_{1}, \widetilde{P}_{2}}(x, f)-k_{\widetilde{P}_{1}, \widetilde{P}_{2}}\left(x, \mathcal{F}_{\mathfrak{t}}(f)\right)\right)\right) \eta_{s}(\operatorname{det} x) d x .
$$

Fixons $\varepsilon_{0}>0$. En utilisant le théorème 2.7 pour $f$ et $\mathcal{F}_{\mathfrak{t}}(f)$ on a donc pour tout $N>0$

$$
\left|I^{T}\left(\eta_{s}, f\right)-I^{T}\left(\eta_{s}, \mathcal{F}_{\mathfrak{t}}(f)\right)\right|=O\left(e^{-N\|T\|}\right)
$$

si $T \in T_{+}+\mathfrak{a}_{0}^{+}$est tel que $\forall \alpha \in \Delta_{\widetilde{B}_{0}}, \alpha(T)>\varepsilon_{0}\|T\|$. D'après la proposition 3.7 la différence $I^{T}\left(\eta_{s}, f\right)-I^{T}\left(\eta_{s}, \mathcal{F}_{\mathfrak{t}}(f)\right)$ est un polynôme-exponentielle en $T$ qui pour $-1<\operatorname{Re}(s)<1$, en vertu du lemme $3.2 b$ ), est soit constant, soit sa norme tend vers $\infty$ quand le paramètre $T \in \mathfrak{a}_{0}^{+}$est tel que $\alpha(T)$ tendent vers $\infty$ pour tout $\alpha \in \Delta_{\widetilde{B}_{0}}$. L'égalité ci-dessus implique alors $I^{T}\left(\eta_{s}, f\right)=$ $I^{T}\left(\eta_{s}, \mathcal{F}_{\mathfrak{t}}(f)\right)$. En vertu de la remarque 3.10 on obtient donc l'égalité des fonctions méromorphes en la variable $s \in \mathbb{C} I^{T}\left(\eta_{s}, f\right)=I^{T}\left(\eta_{s}, \mathcal{F}_{\mathfrak{t}}(f)\right)$. En particulier, en vertu du théorème 3.8, on a égalité de leur termes constants pour $s \neq-1,1$.

\section{$5 \quad$ Orbites semi-simples}

Soit $\mathcal{O}_{r s} \subseteq \mathcal{O}$ l'ensemble des classes contenant un élément $\left(\begin{array}{ll}B & u \\ v & d\end{array}\right)$ tel que le polynôme caractéristique de $B$ est séparable (i.e. $B$ est semi-simple régulier dans $\mathfrak{g}(\mathrm{F})$ ). On appelle de telles classes relativement semi-simples régulières. Si $\mathfrak{o} \in \mathcal{O}_{r s}$, alors tout élément $\left(\begin{array}{cc}B_{0} & u \\ v & d\end{array}\right) \in \mathfrak{o}$ a la propriété que $B_{0}$ soit semi-simple régulier.

Soit $\mathfrak{o} \in \mathcal{O}_{r s}$. Le but de cette section est de donner une expression explicite pour $I_{\mathfrak{o}}\left(\eta_{s}, \cdot\right)$ ce qu'on achève par le théorème 5.12. Les résultats sont analogues, et parfois même identiques, à ceux de la section 5 de [Zyd15a] et par conséquent on renvoie souvent à cet article pour les preuves détaillées. En particulier, on omets les résultats de convergence, qui sont démontrés dans le paragraphe 5.7, ainsi que les résultats de prolongement méromorphe qui se trouvent dans le paragraphe 5.8 de loc. cit. On utilisera aussi la même notation que dans la section 5 de loc. cit. ce qui rendra l'analogie plus visible.

Voici le plan de la section : après avoir introduit quelques notations dans le paragraphe suivant 5.1. on décrit la décomposition de $\mathfrak{o}$ en $G(\mathrm{~F})$-orbites dans le paragraphe 5.2. On introduit encore un peu plus de notation dans 5.3. Dans le paragraphe 5.4 on définit une expression $i_{\mathfrak{o}}(x)$ pour laquelle on a

$$
I_{\mathfrak{o}}\left(\eta_{s}, f\right)=\int_{[G]} i_{\mathfrak{o}}(x) \eta_{s}(\operatorname{det} x) d x
$$

si $-1<\operatorname{Re}(s)<1$. En supposant cela, on donne la preuve du théorème 5.12 omettant les preuves des énoncés techniques. Dans la section 5.5 on introduit un nouveau noyau tronqué $i_{f, \mathfrak{o}}^{T}(x)$ tel que $\int_{[G]} i_{f, \mathfrak{o}}^{T}(x) \eta_{s}(\operatorname{det} x) d x=I_{\mathfrak{o}}^{T}\left(\eta_{s}, f\right)$ pour tout $s \in \mathbb{C}$. Ce résultat nous permet de démontrer (5.1) dans le paragraphe suivant 5.6. On finit la preuve dans le dernier paragraphe 5.7 où on étudie certaines fonctions zêtas - les homologues des fonctions zêtas étudies dans [Zyd15a].

\subsection{Notations}

On utilisera les lettres $I, J$, avec de possibles indices, pour noter des sous-ensembles finis de $\mathbb{N}^{*}$. Soit $I \subseteq \mathbb{N}^{*}$ fini. On pose $-I=\bigcup_{i \in I}\{-i\}$. On dit que $\mathcal{I}$ est un $\epsilon$-sous-ensemble de $I$, si $\mathcal{I} \subseteq I \cup-I$ et si pour tout $i \in \mathcal{I}$ on a $-i \notin \mathcal{I}$. Dans ce cas on écrit $\mathcal{I} \subseteq \subseteq_{\epsilon} I$. La notation est un peu abusive car $\mathcal{I}$ n'est pas forcément un sous-ensemble de $I$. On définit aussi $|\mathcal{I}|=\{|i| \mid i \in \mathcal{I}\} \subseteq \mathbb{N}^{*}$ et $\mathcal{I}^{\sharp} \subseteq_{\epsilon} I$ par la propriété $\mathcal{I} \cup \mathcal{I}^{\sharp}=|\mathcal{I}| \cup-|\mathcal{I}|$. Autrement dit $\mathcal{I}^{\sharp}=-\mathcal{I}$ mais on écrira $\mathcal{I}^{\sharp}$ dans ce 
contexte. On réserve les lettres $\mathcal{I}, \mathcal{J}$ et $\mathcal{K}$ et seulement ces trois lettres avec de possibles indices, pour des $\epsilon$-sous-ensembles.

On utilisera aussi la notation abrégée suivante soit $I^{\prime} \subseteq \mathbb{N}^{*}$ et $\mathcal{I}, \mathcal{J} \subseteq_{\epsilon} I^{\prime}$, on écrira $\mathcal{J} \cup \mathcal{I} \subseteq{ }_{\epsilon} I^{\prime}$ pour signifier que la réunion ensembliste $\mathcal{I} \cup \mathcal{J}$ est aussi un $\epsilon$-sous-ensemble (ce qui n'est pas toujours vrai). On utilise le symbole $\sqcup$ pour noter la réunion disjointe, donc $I \sqcup J=I^{\prime}$ implique $I \cap J=\varnothing$. On écrira aussi, pour $\mathcal{I} \subseteq \subseteq_{\epsilon} I^{\prime}$ fixés

$$
\sum_{|\mathcal{J}|=I^{\prime}}:=\sum_{\substack{\mathcal{J} \subseteq \epsilon \\|\mathcal{J}|=I^{\prime}}}, \sum_{\mathcal{K} \sqcup \mathcal{J} \subseteq \mathcal{I}}:=\sum_{\substack{\mathcal{K}, \mathcal{J} \subseteq \mathcal{I} \\ \mathcal{K} \cap \mathcal{J}=\varnothing}}, \sum_{|\mathcal{K}| \sqcup|\mathcal{J}|=I^{\prime}}:=\sum_{\substack{\mathcal{K}, \mathcal{J} \subseteq \epsilon I^{\prime} \\|\mathcal{K}| \sqcup|\mathcal{J}|=I^{\prime}}} .
$$

Finalement, pour $\mathcal{J}_{1} \sqcup \mathcal{J}_{3} \sqcup \mathcal{J}_{4} \subseteq_{\epsilon} I_{0}$ et $\mathcal{J}_{2} \subseteq \mathcal{J}_{3}$ on utilisera parfois la notation suivante

$$
\mathcal{J}_{3 \backslash 2}:=\mathcal{J}_{3} \backslash \mathcal{J}_{2}, \mathcal{J}_{13}:=\mathcal{J}_{1} \cup \mathcal{J}_{3}, \mathcal{J}_{134}:=\mathcal{J}_{1} \cup \mathcal{J}_{3} \cup \mathcal{J}_{4} \text { etc. }
$$

\subsection{Orbites dans une classe relativement semi-simple régulière}

Dans ce paragraphe on décrit une décomposition en orbites d'une classe relativement semi-simple régulière.

En utilisant la décomposition (2.1) on va écrire les éléments $X$ de $\widetilde{\mathfrak{g}}$ sous forme

$$
X=(B, \mathrm{w}, d)
$$

où $B \in \mathfrak{g}, \mathrm{w} \in V \times V^{*}$ et $d \in \mathbb{G}_{a}$. On considère alors $V \times V^{*}$ comme un espace vectoriel dont $V$ et $V^{*}$ sont des sous-espaces. L'action d'un $g \in G$ s'écrit donc simplement

$$
\operatorname{Ad}(g) X=(\operatorname{Ad}(g) B, g \mathrm{w}, d)
$$

où $G$ agit sur $V \times V^{*}$ par ses actions naturelles sur $V$ et $V^{*}$.

Donnons-nous

- Un polynôme séparable $Q \in \mathrm{F}[T]$ de degré $n$.

- Une décomposition de $Q$ en facteurs irréductibles $Q=\prod_{i=1}^{m} Q_{i}$ et notons $I=\{1, \ldots, m\}$.

- Pour tout $i \in I$ notons $\mathrm{F}_{i}=\mathrm{F}[T] /\left(Q_{i}(T)\right)\left(\right.$ resp. $\left.\mathrm{F}_{i}=\mathrm{F}[T] /(-1) \operatorname{deg} Q_{i} Q_{i}(-T)\right)$ et $F_{I}=$ $\mathrm{F}[T] /(Q(T))\left(\right.$ resp. $F_{I}=\mathrm{F}[T] /(-1)^{n}(Q(T))$ ). On identifie $\mathrm{F}_{I}$ (resp. $\mathrm{F}_{-I}$ ) à $\prod_{i \in I} \mathrm{~F}_{i}$ (resp. à $\left.\prod_{i \in I} \mathrm{~F}_{-i}\right)$ à l'aide des homomorphismes des F-algèbres $\mathrm{F}[T] /(Q(T))=\mathrm{F}_{I} \rightarrow \mathrm{F}_{i}=$ $\mathrm{F}[T] /\left(Q_{i}(T)\right)\left(\right.$ resp. $\left.\mathrm{F}_{-I} \rightarrow \mathrm{F}_{-i}\right)$ induits par $T \mapsto T$.

- On fixe l'isomorphisme des algèbres étales $\iota_{I}: \mathrm{F}_{I} \rightarrow \mathrm{F}_{-I}$ induit par $T \mapsto-T$. On identifie $\mathrm{F}_{-I}$ à $\mathrm{F}_{I}^{*}$, en tant que $\mathrm{F}$-espace vectoriel grâce à la forme bilinéaire non-dégénérée $\mathrm{F}_{I} \times$ $\mathrm{F}_{-I} \ni(u, v) \mapsto \operatorname{Tr}_{\mathrm{F}_{I} / \mathrm{F}}\left(u \iota_{I}(v)\right)$.

- On note $b_{I} \in \mathrm{F}_{I}$ l'image de $T$ dans $\mathrm{F}_{I}$. Dans ce cas $b_{I}$ engendre la $\mathrm{F}$-algèbre étale $\mathrm{F}_{I}$.

Soit alors $P_{I}=M_{I} N_{I}$ le sous-groupe parabolique standard de $G$ défini comme le stabilisateur du drapeau

$$
0=V_{j_{0}} \subsetneq V_{j_{1}} \subsetneq V_{j_{2}} \subsetneq \cdots \subsetneq V_{j_{m}}=V
$$

où pour $i \in I$ on a $\operatorname{deg} Q_{i}=j_{i}-j_{i-1}$. Pour $i \in I$ posons $Z_{i}:=\bigoplus_{j=j_{i-1}+1}^{j_{i}} D_{j}$. On peut, et on fixe un $B \in \mathfrak{m}_{P_{I}}(\mathrm{~F})$ dont $Q_{i}$ est le polynôme caractéristique pour son action sur $Z_{i}$. Alors, $B$ est semi-simple régulier dans $\mathfrak{g}(\mathrm{F})$ et tout élément semi-simple régulier de $\mathfrak{g}(\mathrm{F})$ s'obtient par une telle construction.

L'élément $B$ induit une structure de $\mathrm{F}[T]$-module sur $V$ par $T \cdot v=B v$. L'algèbre $\mathrm{F}_{I}$ est aussi naturellement un $\mathrm{F}[T]$-module qui est isomorphe, en tant que $\mathrm{F}[T]$-module à $V$. On fixe un tel isomorphisme et on identifie $V$ avec $\mathrm{F}_{I}$ désormais. Notons qu'un tel isomorphisme identifie 
aussi $\mathrm{F}_{i}$ avec $Z_{i}$ pour tout $i \in \mathrm{I}$. En plus, l'isomorphisme $V \cong \mathrm{F}_{I}$ induit l'isomorphisme duale entre $V^{*}$ et $\left(\mathrm{F}_{I}\right)^{*}=\mathrm{F}_{-I}$. On identifie aussi ces espaces. On a donc pour tous $(u, v) \in \mathrm{F}_{I} \times \mathrm{F}_{-I}$, $g \in G(\mathrm{~F})$ et $k \in \mathbb{N}$ :

$$
\begin{gathered}
v\left(B^{k} u\right)=\operatorname{Tr}_{\mathrm{F}_{I} / \mathrm{F}}\left(b_{I}^{k} u \iota_{I}(v)\right), \\
\operatorname{Tr}_{\mathrm{F}_{I} / \mathrm{F}}\left(g u \iota_{I}(g v)\right)=\operatorname{Tr}_{\mathrm{F}_{I} / \mathrm{F}}\left(u \iota_{I}(v)\right) .
\end{gathered}
$$

Pour tout $i \in I$ on note $1_{i}$ et $1_{-i}$ les unités de $\mathrm{F}_{i}$ et $\mathrm{F}_{-i}$ respectivement. Pour tout $\mathcal{I} \subseteq \subseteq_{\epsilon} I$ on pose $\mathrm{F}_{\mathcal{I}}=\bigoplus_{i \in I} \mathrm{~F}_{i} \subseteq \mathrm{F}_{I} \times \mathrm{F}_{-I}$ et $1_{\mathcal{I}}=\sum_{i \in I} 1_{i} \in \mathrm{F}_{\mathcal{I}}^{*}$.

Soit $T_{I}$ le centralisateur de $B$ dans $G$. C'est un F-tore maximal de $G$, et il est contenu dans $M_{I}$. Pour $J \subseteq I$ on note $T_{J} \subseteq T_{I}$ le plus grand sous-tore qui agit trivialement sur $\mathrm{F}_{I \backslash J}$. Si $J=\{i\}$ on écrit simplement $T_{i}=T_{\{i\}}$. Alors $T=\prod_{i \in I} T_{i}$ et pour tout $i \in I$ le groupe $T_{i}(\mathrm{~F})$ agit simplement transitivement sur $\mathrm{F}_{i}^{*}$ ainsi que sur $\mathrm{F}_{-i}^{*}$.

On fixe une classe $\mathfrak{o} \in \mathcal{O}_{r s}$ contenant un élément $X$ de type $(B, \mathrm{w}, d)$. Remarquons que $d$ est le même pour tout $X \in \mathfrak{o}$, on le note simplement $d_{\mathfrak{o}}$.

On introduit l'ensemble $\mathrm{V}_{\mathfrak{o}}=\left\{(u, v) \in \mathrm{F}_{I} \times \mathrm{F}_{-I} \mid \operatorname{Tr}_{\mathrm{F}_{I} / \mathrm{F}}\left(b_{I}^{i} u \iota_{I}(v)\right)=A_{i}(X) \forall 0 \leq i \leq n-1\right\}$ où $X \in \mathfrak{o}$ quelconque. Comme $T_{I}(\mathrm{~F})$ agit sur $F_{I} \times \mathrm{F}_{-I}$, commute à $B$ et préserve l'accouplement naturel sur $\mathrm{F}_{I} \times \mathrm{F}_{-I}$, il agit aussi sur $\mathrm{V}_{\mathfrak{o}}$. On voit que l'ensemble des orbites dans $\mathrm{V}_{\mathfrak{o}}$ sous l'action de $T_{I}(\mathrm{~F})$ est en bijection avec l'ensemble des classes de $G(\mathrm{~F})$-conjugaison dans $\mathfrak{o}$, la bijection étant induite par l'application $\mathrm{V}_{\mathfrak{o}} \ni \mathrm{w} \mapsto\left(B, \mathrm{w}, d_{\mathfrak{o}}\right)$.

Pour $\mathrm{w} \in \mathrm{F}_{I} \times \mathrm{F}_{-I}$ et $\mathcal{I} \subseteq_{\epsilon} I$ on pose $\mathrm{w}_{\mathcal{I}}:=1_{\mathcal{I}} \mathrm{w}$. Si $\mathcal{I}=\{i\}$ on note simplement $\mathrm{w}_{i}=\mathrm{w}_{\{i\}}$. On va continuer pourtant d'écrire parfois les éléments de $\mathrm{F}_{I} \times \mathrm{F}_{-I}$ comme $(u, v)$.

Lemme 5.1. Il existe un $\alpha_{I} \in \mathrm{F}_{I}$ tel que pour tout $(u, v) \in \mathrm{F}_{I} \times \mathrm{F}_{-I}$ on a

$$
(u, v) \in \mathrm{V}_{\mathfrak{o}} \Longleftrightarrow u \iota_{I}(v)=\alpha_{I} .
$$

Démonstration. Pour tous $(u, v),\left(u^{\prime}, v^{\prime}\right) \in \mathrm{V}_{\mathfrak{o}}$ et $k \in \mathbb{N}$ on a

$$
v\left(B^{k} u\right)=\operatorname{Tr}_{\mathrm{F}_{I} / \mathrm{F}}\left(b_{I}^{k} u \iota_{I}(v)\right)=\operatorname{Tr}_{\mathrm{F}_{I} / \mathrm{F}}\left(b_{I}^{k} u^{\prime} \iota_{I}\left(v^{\prime}\right)\right)=v^{\prime}\left(B^{k} u^{\prime}\right)
$$

d'où

$$
\operatorname{Tr}_{\mathrm{F}_{I} / \mathrm{F}}\left(b_{I}^{k}\left(u \iota_{I}(v)-u^{\prime} \iota_{I}\left(v^{\prime}\right)\right)\right)=0, \quad \forall k \in \mathbb{N} .
$$

D'après la proposition (18.3) dans [KMRT98, la forme $\operatorname{Tr}_{\mathrm{F}_{I} / \mathrm{F}}$ est non-dégénérée, et puisque les puissances de $b_{I}$ engendrent $\mathrm{F}_{I}$ sur $\mathrm{F}$ on obtient

$$
u \iota_{I}(v)=u^{\prime} \iota_{I}\left(v^{\prime}\right), \quad \forall(u, v),\left(u^{\prime}, v^{\prime}\right) \in \mathrm{V}_{\mathfrak{o}} .
$$

On pose donc $\alpha_{I}:=u \iota_{I}(v)$ où $(u, v) \in \mathrm{V}_{\mathfrak{o}}$ quelconque. Il reste à démontrer que si $(u, v) \in \mathrm{V}_{\mathfrak{o}}$ est tel que $u \iota_{I}(v)=\alpha_{I}$ alors $(u, v) \in \mathrm{V}_{\mathfrak{o}}$. Pour cela il suffit de faire le même calcul dans le sens inverse.

Soit $\alpha_{I}=\left(\alpha_{i}\right)_{i \in I} \in \mathrm{F}_{I}$ comme dans le lemme précédant. Notons $I_{0} \subseteq I$ l'ensemble de $i \in I$ tels que $\alpha_{i}=0$.

Proposition 5.2. Il existe une unique $T_{I}(\mathrm{~F})$-orbite dans $\mathrm{V}_{\mathfrak{o}}$ composée des $(u, v) \in \mathrm{V}_{\mathfrak{o}}$ tels que $u_{i}=0$ et $v_{-i}=0$ pour tout $i \in I_{0}$. On choisit $\xi_{\varnothing}$ un représentant de cette orbite. Alors, les $T_{I}(\mathrm{~F})$-orbites dans $\mathrm{V}_{\mathfrak{o}}$ sont en bijection avec les $\epsilon$-sous-ensembles de $I_{0}$, le représentant de l'orbite correspondant à $\mathcal{I} \subseteq_{\epsilon} I_{0}$ étant $\xi_{\varnothing}+1_{\mathcal{I}}$. Les orbites de dimension maximale correspondent aux $\mathcal{I} \subseteq I_{\epsilon}$ tels que $|\mathcal{I}|=I_{0}$.

Démonstration. La preuve est identique à celle de la proposition 5.2 de [Zyd15a]. 


\subsection{Quelques définitions associées aux orbites}

D'après la proposition 5.2 ci-dessus les

$$
X_{\mathcal{I}}:=\left(B, \xi_{\varnothing}+1_{\mathcal{I}}, d_{\mathfrak{o}}\right)
$$

où $\mathcal{I} \subseteq_{\epsilon} I_{0}$, sont des représentants des orbites pour l'action de $G(\mathrm{~F})$ à $\mathfrak{o}$. On les considère fixés désormais.

Pour un sous-F-groupe $H$ de $G, X \in \widetilde{\mathfrak{g}}(\mathrm{F})$ et une F-algèbre $R$, notons $H(R, X)$ le groupe des $R$-points du stabilisateur de $X$ dans $H$. Alors, pour tout $\mathcal{I} \subseteq_{\epsilon} I_{0}$ et toute $\mathrm{F}$-algèbre $R$ on a :

$$
G\left(R, X_{\mathcal{I}}\right)=T_{I_{0} \backslash|\mathcal{I}|}(R) .
$$

Pour tout $i \in I$ on choisit une mesure de Haar sur $T_{i}(\mathbb{A})$ et pour tout $I^{\prime} \subseteq I$ on met la mesure produit sur $T_{I^{\prime}}(\mathbb{A})=\prod_{i \in I^{\prime}} T_{i}(\mathbb{A})$. On note $H_{I}$ l'application de Harish-Chandra, introduite dans le paragraphe 1.1. par rapport au sous-groupe parabolique standard $P_{I}$. Pour $I^{\prime} \subseteq I$ on note $\mathfrak{a}_{I^{\prime}}$ l'image et $T_{I^{\prime}}(\mathbb{A})^{1}$ le noyau de la restriction de l'application $H_{I}$ à $T_{I^{\prime}}(\mathbb{A})$ (la restriction étant un homomorphisme car $T_{I^{\prime}}(\mathbb{A}) \subseteq M_{P_{I}}(\mathbb{A})$ ). En particulier $\mathfrak{a}_{I}=\mathfrak{a}_{P_{I}}$ et le quotient $T_{I^{\prime}}(\mathrm{F}) \backslash T_{I^{\prime}}(\mathbb{A})^{1}$ est compact. Puisque $H_{I}$ restreint à $T_{I^{\prime}}(\mathbb{A}) \cap A_{P_{I}}^{\infty}$ est un isomorphisme on obtient une unique mesure de Haar sur $T_{I^{\prime}}(\mathbb{A})^{1}$ compatible avec celles sur $T_{I^{\prime}}(\mathbb{A})$ et $\mathfrak{a}_{I^{\prime}}$.

Si $I^{\prime \prime} \subseteq I^{\prime} \subseteq I$, les espaces $\mathfrak{a}_{I^{\prime \prime}}$ et $\mathfrak{a}_{I^{\prime} \backslash I^{\prime \prime}}$ sont en somme directe. On voit alors $\mathfrak{a}_{I^{\prime}}^{*}:=$ $\operatorname{Hom}_{\mathbb{R}}\left(\mathfrak{a}_{I^{\prime}}, \mathbb{R}\right)$ comme un sous-espace de $\mathfrak{a}_{0}^{*}$, et donc de $\mathfrak{a}_{\tilde{0}}^{*}$ aussi.

En particulier, puisque $A_{P_{I}} \subseteq T_{I} \subseteq M_{P_{I}}$, on voit que $\mathfrak{a}_{I}^{*}$ s'identifie à $\operatorname{Hom}_{\mathrm{F}}\left(T_{I}, \mathbb{G}_{m}\right) \otimes_{\mathbb{Z}} \mathbb{R}$. Pour tout $i \in I \cup-I$ on note alors $\rho_{i} \in \mathfrak{a}_{I}^{*}$ le caractère par lequel $T_{I}$ agit sur $\mathrm{F}_{i}$. On a alors $\rho_{i}=-\rho_{-i}$. Soit $\mathcal{I} \subseteq_{\epsilon} I_{0}$. On a donc que $\left\{\rho_{i}\right\}_{i \in \mathcal{I}}$ est une base de $\mathfrak{a}_{|\mathcal{I}|}^{*}$. On pose aussi

$$
\rho_{\mathcal{I}}=\sum_{i \in \mathcal{I}} \rho_{i} \in \mathfrak{a}_{|\mathcal{I}|}^{*}
$$

Notons que $\rho_{\mathcal{I}^{\sharp}}=-\rho_{\mathcal{I}}$.

On définit encore :

- pour $I^{\prime} \subseteq I$, l'espace $\mathfrak{a}_{I^{\prime}, \mathbb{C}}^{*}:=\mathfrak{a}_{I^{\prime}}^{*} \otimes_{\mathbb{R}} \mathbb{C}$;

- pour $\mathcal{I} \subseteq_{\epsilon} I_{0}, \mathbb{1}_{\mathcal{I}}$ la fonction caractéristique de $H \in \mathfrak{a}_{I_{0}}$ tels que

$$
\rho_{i}(H) \leq 0, \forall i \in \mathcal{I} \cap I_{0} \quad \text { et } \quad \rho_{i}(H)<0, \forall i \in \mathcal{I} \cap-I_{0}
$$

\subsection{Le résultat principal}

Dans ce paragraphe on énonce et on démontre le théorème 5.12. Cependant, certains résultats seront seulement énoncés avec les renvois vers leurs démonstrations dans les paragraphes suivants.

On note $M_{\widetilde{I}}$ le sous-groupe de Levi de $\widetilde{G}$ stabilisant $\mathrm{F}_{i}$ pour tout $i \in I$ ainsi que $D_{0}$ et l'on note $M_{\widetilde{I}_{0}}$ le plus grand sous-groupe de Levi de $\widetilde{G}$ qui stabilise les espaces $\mathrm{F}_{i}$ pour tout $i \in I_{0}$. On a donc $M_{\widetilde{I}} \subseteq M_{\widetilde{I}_{0}}$.

Soit $\widetilde{Q} \in \mathcal{F}\left(M_{\widetilde{I}}\right)$, remarquons alors qu'il existe un unique $\mathcal{I} \subseteq_{\epsilon} I$ tel que l'espace $\mathcal{V}_{\widetilde{Q}}$ défini dans le paragraphe 1.6 égale $\mathrm{F}_{\mathcal{I}}$. On pose dans ce cas $\mathcal{I}_{\widetilde{Q}}=\mathcal{I}$. D'ailleurs, il est clair que si l'orbite d'un $X_{\mathcal{I}}$ (voir (5.3) ) où $\mathcal{I} \subseteq_{\epsilon} I_{0}$ intersecte non-trivialement $\mathfrak{m}_{\widetilde{P}}(\mathrm{~F})$ où $\widetilde{P} \in \mathcal{F}\left(M_{\widetilde{0}}, B\right)$ alors celui-ci est conjugué à un élément de $\mathcal{F}\left(M_{\widetilde{I}}\right)$. On peut alors reformuler le lemme 2.3 ainsi :

Lemme 5.3. Soit $\widetilde{Q} \in \mathcal{F}\left(M_{\widetilde{I}}\right)$. Alors $X_{\mathcal{I}} \in \mathfrak{m}_{\widetilde{Q}}(\mathrm{~F})$ si et seulement si $\left(|\mathcal{I}| \cup\left(I \backslash I_{0}\right)\right) \cap\left|\mathcal{I}_{\widetilde{Q}}\right|=\varnothing$. 
On a en particulier donc que si $\mathfrak{m}_{\widetilde{Q}}(\mathrm{~F}) \cap \mathfrak{o} \neq \varnothing$, alors $\left(I \backslash I_{0}\right) \cap\left|\mathcal{I}_{\widetilde{Q}}\right|=\varnothing$ autrement dit $\widetilde{Q} \in \mathcal{F}\left(M_{\widetilde{I}_{0}}\right)$.

Pour un sous-groupe parabolique relativement standard $\widetilde{P}$ de $\widetilde{G}$ on pose

$$
\mathcal{F}\left(M_{\widetilde{I}_{0}}, \widetilde{P}\right)^{r e l}=\left\{\widetilde{Q} \in \mathcal{F}\left(M_{\widetilde{I}_{0}}\right) \mid \exists \gamma \in G(\mathrm{~F}), \gamma \widetilde{Q} \gamma^{-1}=\widetilde{P}\right\} .
$$

Soit $\widetilde{Q} \in \mathcal{F}\left(M_{\widetilde{I_{0}}}, \widetilde{P}\right)^{r e l}$ et $\gamma \in G(\mathrm{~F})$ tel que $\gamma \widetilde{Q} \gamma^{-1}=\widetilde{P}$. Grâce à la décomposition de Bruhat, on choisi alors un $s_{\widetilde{Q}} \in \Omega^{G} / \Omega^{Q}$ tel que $\gamma \in B(\mathrm{~F}) w_{s_{\widetilde{Q}}} Q(\mathrm{~F})$. On a alors $w_{s_{\widetilde{Q}}} \widetilde{Q} w_{s_{\widetilde{Q}}}^{-1}=\widetilde{P}$.

On a alors :

Lemme 5.4. Soient $\widetilde{P} \in \mathcal{F}\left(M_{\widetilde{0}}, B\right)$ et $\mathcal{I} \subseteq_{\epsilon} I_{0}$. Alors, l'intersection de la $G(\mathrm{~F})$-orbite de $X_{\mathcal{I}}$ avec $\mathfrak{m}_{\widetilde{P}}(\mathrm{~F})$ égale

$$
\coprod_{\substack{\widetilde{Q} \in \mathcal{F}\left(M_{\widetilde{I}}, \widetilde{P}\right)^{r e l} \\|\mathcal{I}| \cap\left|\mathcal{I}_{\widetilde{Q}}\right|=\varnothing}} \coprod_{\delta \in M_{P}\left(\mathrm{~F}, \operatorname{Ad}\left(w_{\widetilde{Q}_{\widetilde{Q}}}\right) X_{\mathcal{I}}\right) \backslash M_{P}(\mathrm{~F})}\left\{\operatorname{Ad}\left(\delta^{-1} w_{s_{\widetilde{Q}}}\right) X_{\mathcal{I}}\right\} .
$$

Démonstration. Voir la preuve du lemme 5.4 de Zyd15a.

Pour une fonction $\phi$ sur $\widetilde{\mathfrak{g}}(\mathbb{A})$ et $x \in G(\mathbb{A})$ on définit

$$
\phi_{x}(X):=\phi\left(\operatorname{Ad}\left(x^{-1}\right) X\right) .
$$

Fixons $f \in \mathcal{S}(\widetilde{\mathfrak{g}}(\mathbb{A}))$. Soit $\widetilde{P}$ un sous-groupe parabolique semi-standard de $\widetilde{G}$. D'après le lemme $2.3 \mathrm{~b}$ ) on a un isomorphisme $N_{P}$-équivariant

$$
\mathfrak{n}_{\widetilde{P}} \cong \mathfrak{n}_{P} \oplus \mathcal{V}_{\widetilde{P}}
$$

On pose

$$
f^{\widetilde{P}}(X)=\int_{\mathcal{V}_{\widetilde{P}}(\mathbb{A})} f(X+Y) d Y, \quad X \in \widetilde{\mathfrak{g}}(\mathbb{A}) .
$$

Supposons en plus que $\widetilde{P}$ est relativement standard et posons

$$
i_{\widetilde{P}, \mathfrak{o}}(x)=i_{f, \widetilde{P}, \mathfrak{o}}(x)=\sum_{\xi \in \mathfrak{m}_{\widetilde{P}}(\mathrm{~F}) \cap \mathfrak{o}} \sum_{\gamma \in N_{P}(\mathrm{~F})} f_{\gamma x}^{\widetilde{P}}(\xi), \quad x \in P(\mathrm{~F}) \backslash G(\mathbb{A}),
$$

où $f_{x}^{\widetilde{P}}=\left(f_{x}\right)^{\widetilde{P}}$. En vertu du lemme 5.4 on a alors

$$
i_{\widetilde{P}, \mathfrak{o}}(x)=\sum_{\widetilde{Q} \in \mathcal{F}\left(M_{\widetilde{I_{0}}}, \widetilde{P}\right)^{r e l}} \sum_{\substack{\mathcal{I} \subset_{\epsilon} I_{0} \\|\mathcal{I}| \cap\left|\mathcal{I}_{\widetilde{Q}}\right|=\varnothing}} \sum_{\gamma \in P\left(\mathrm{~F}, \operatorname{Ad} w_{\widetilde{Q}} X_{\mathcal{I}}\right) \backslash P(\mathrm{~F})} f_{\gamma x}^{\widetilde{P}}\left(\operatorname{Ad}\left(w_{\widetilde{Q}}\right) X_{\mathcal{I}}\right) .
$$

On voit donc que

$$
\sum_{\delta \in P(\mathrm{~F}) \backslash G(\mathrm{~F})} i_{\widetilde{P}, \mathfrak{o}}(\delta x)=\sum_{\widetilde{Q} \in \mathcal{F}\left(M_{\widetilde{I_{0}}}, \widetilde{P}\right)^{r e l}} \sum_{\substack{\mathcal{I} \in_{\epsilon} I_{0} \\|\mathcal{I}| \cap\left|\mathcal{I}_{\widetilde{Q}}\right|=\varnothing}} \sum_{\delta \in G\left(\mathrm{~F}, X_{\mathcal{I}}\right) \backslash G(\mathrm{~F})} f_{\delta x}^{\widetilde{Q}}\left(X_{\mathcal{I}}\right) .
$$

On expliquera bientôt pourquoi la somme ci-dessus converge.

Pour tout $\mathcal{J} \subseteq_{\epsilon} I_{0}$ posons $\mathbb{A}_{\mathcal{J}}=\mathbb{A} \otimes_{\mathrm{F}} \mathrm{F}_{\mathcal{J}}$. Notons aussi $\mathbb{A}_{I^{\prime}}=\mathbb{A} \otimes_{\mathrm{F}} \mathrm{F}_{I^{\prime}}$ et $\mathbb{A}_{-I^{\prime}}=\mathbb{A} \otimes_{\mathrm{F}} \mathrm{F}_{-I^{\prime}}$ pour tout $I^{\prime} \subseteq I$. Fixons un caractère additif continu non-trivial $\psi$ sur $\mathrm{F} \backslash \mathbb{A}$. Pour une fonction $\phi \in \mathcal{S}(\widetilde{\mathfrak{g}}(\mathbb{A}))$ et $\mathcal{J} \subseteq_{\epsilon} I_{0}$ on définit la "transformée de Fourier" de $\phi$ par rapport à $\mathcal{J}$

$$
\hat{\phi}^{\mathcal{J}}(X)=\int_{\mathbb{A}_{\mathcal{J}}} \phi\left(B^{\prime}, u_{\mathcal{J}}+\mathrm{w}^{\prime}-\mathrm{w}_{\mathcal{J}^{\sharp}}^{\prime}, d^{\prime}\right) \psi\left(\left\langle u_{\mathcal{J}}, \mathrm{w}_{\mathcal{J}^{\sharp}}^{\prime}\right\rangle\right) d u_{\mathcal{J}}
$$


où $X=\left(B^{\prime}, \mathrm{w}^{\prime}, d^{\prime}\right) \in \widetilde{\mathfrak{g}}(\mathbb{A}), d u_{\mathcal{J}}$ c'est la mesure de Haar sur $\mathbb{A}_{\mathcal{J}}$ pour laquelle $\mathrm{F}_{\mathcal{J}} \backslash \mathbb{A}_{\mathcal{J}}$ est de volume 1 et $\langle\cdot, \cdot\rangle$ c'est l'accouplement défini par la forme trace. En fait, si l'on écrit $\mathrm{w}^{\prime}=\left(u^{\prime}, v^{\prime}\right) \in$ $\mathbb{A}_{I} \times \mathbb{A}_{-I}$ et $u_{\mathcal{J}}=(u, v)$ où $u \in \mathbb{A}_{\mathcal{J} \cap I}$ et $v \in \mathbb{A}_{\mathcal{J} \cap-I}$ on a

$$
\left\langle u_{\mathcal{J}}, \mathrm{w}_{\mathcal{J}^{\sharp}}^{\prime}\right\rangle=\left\langle u_{\mathcal{J}}, \mathrm{w}^{\prime}\right\rangle=\operatorname{Tr}_{\mathrm{F}_{I} / \mathrm{F}}\left(u^{\prime} \iota_{I}(v)+u_{I}\left(v^{\prime}\right)\right) .
$$

La restriction de la fonction $\hat{\phi}^{\mathcal{J}}$ à $\mathfrak{g}(\mathbb{A}) \times \mathbb{A}_{I \backslash(\mathcal{J} \cap I)} \times \mathbb{A}_{-I \backslash(\mathcal{J} \cap-I)} \times \mathbb{A}$ est alors de type BruhatSchwartz. Dans tous ce qui suit les arguments des fonctions de type $\hat{\phi}^{\mathcal{J}}$ seront toujours dans $\mathfrak{g}(\mathbb{A}) \times \mathbb{A}_{I \backslash(\mathcal{J} \cap I)} \times \mathbb{A}_{-I \backslash(\mathcal{J} \cap-I)} \times \mathbb{A}$ de façon qu'on pourrait les traiter comme les fonctions de type Bruhat-Schwartz.

Soient alors $\widetilde{Q} \in \mathcal{F}\left(M_{\widetilde{I_{0}}}, \widetilde{P}\right)^{r e l}$ et $\mathcal{I} \subseteq_{\epsilon} I_{0}$ tel que $|\mathcal{I}| \cap\left|\mathcal{I}_{\widetilde{Q}}\right|=\varnothing$. On a $f_{x}^{\widetilde{Q}}\left(X_{\mathcal{I}}\right)={\widehat{\left(f_{x}\right.}}^{\mathcal{I}_{\widetilde{Q}}}\left(X_{\mathcal{I}}\right)=$ $\hat{f}_{x}^{\mathcal{I}} \widetilde{Q}\left(X_{\mathcal{I}}\right)$. En utilisant (5.4), on voit donc qu'on peut réécrire (5.8) comme

$$
\sum_{\widetilde{Q} \in \mathcal{F}\left(M_{\widetilde{I}_{0}}, \widetilde{P}\right)^{r e l}} \sum_{\substack{\mathcal{I} \subseteq_{\epsilon} I_{0} \\|\mathcal{I}| \cap\left|\mathcal{I}_{\widetilde{Q}}\right|=\varnothing}} \sum_{\delta \in T_{I_{0} \backslash|\mathcal{I}|}(\mathrm{F}) \backslash G(\mathrm{~F})} \hat{f}_{\delta x}^{\hat{I}_{\widetilde{Q}}}\left(X_{\mathcal{I}}\right) .
$$

En particulier, la somme (5.8) est convergente et par conséquent bien définie.

On pose :

$$
i_{\mathfrak{o}}(x)=i_{f, \mathfrak{o}}(x)=\sum_{\widetilde{P} \in \mathcal{F}\left(M_{\widetilde{0}}, B\right)}(-1)^{d \widetilde{P}_{\widetilde{P}}^{\widetilde{P}}} \sum_{\delta \in P(\mathrm{~F}) \backslash G(\mathrm{~F})} i_{\widetilde{P}, \mathfrak{o}}(\delta x), \quad x \in G(\mathrm{~F}) \backslash G(\mathbb{A}) .
$$

Proposition 5.5 (cf. 5.17). On a pour tout $s \in \mathbb{C}$ tel que $-1<\operatorname{Re}(s)<1$ :

$$
\int_{G(\mathrm{~F}) \backslash G(\mathbb{A})}\left|i_{f, \mathfrak{o}}(x) \eta_{s}(\operatorname{det} x)\right| d x<\infty \quad \text { et } \quad \int_{G(\mathrm{~F}) \backslash G(\mathbb{A})} i_{f, \mathfrak{o}}(x) \eta_{s}(\operatorname{det} x) d x=I_{\mathfrak{o}}\left(\eta_{s}, f\right) .
$$

En utilisant le résultat ci-dessus, on a $\int_{[G]} i_{\mathfrak{o}}(x) \eta_{s}(x) d x=I_{\mathfrak{o}}\left(\eta_{s}, f\right)$ pour $-1<\operatorname{Re}(s)<1$, où, grâce à la formule (5.10), on a

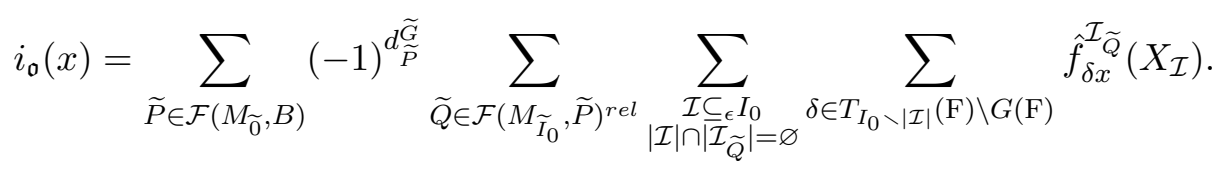

En inversant l'ordre de sommation on a aussi

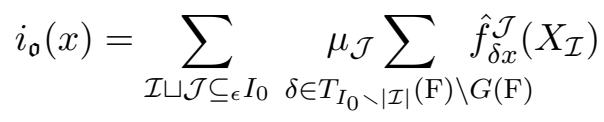

où

$$
\mu_{\mathcal{J}}=\sum_{\widetilde{P} \in \mathcal{F}\left(M_{\widetilde{0}}, B\right)}(-1)^{d d_{\widetilde{P}}^{\widetilde{\mathcal{P}}}} \sum_{\substack{\widetilde{Q} \in \mathcal{F}\left(M_{\widetilde{I_{0}}} \widetilde{P}\right)^{r e l} \\ \mathcal{I}_{\widetilde{Q}}=\mathcal{J}}} 1
$$

Lemme 5.6. Soit $\mathcal{J} \subseteq_{\epsilon} I_{0}$. Alors $\mu_{\mathcal{J}}=(-1)^{\# \mathcal{J}}$.

Démonstration. Remarquons d'abord qu'on a $\mu_{\mathcal{J}}=\sum_{\substack{\widetilde{Q} \in \mathcal{F}\left(M_{\widetilde{I}_{0}}\right) \\ \mathcal{I}_{\widetilde{Q}}=\mathcal{J}^{\prime}}}(-1)^{d d_{\widetilde{Q}}^{\widetilde{G}}}$. Soit $J_{1}=\mathcal{J} \cap I_{0}$ et $J_{2}=-\left(\mathcal{J} \cap-I_{0}\right)$. Soit $\widetilde{Q} \in \mathcal{F}\left(M_{\widetilde{I}_{0}}\right)$ et notons $k=d_{\widetilde{Q}}^{\widetilde{G}}$. Alors, la condition $\mathcal{I}_{\widetilde{Q}}=\mathcal{J}$ veut dire 
qu'il existe des sous-ensembles $\varnothing=I_{0} \subsetneq I_{1} \subsetneq \cdots \subsetneq I_{i}=J_{1}$ et $\varnothing=I_{0}^{\prime} \subsetneq I_{1}^{\prime} \subsetneq \cdots \subsetneq I_{k-i}^{\prime}=J_{2}$ tels que $\widetilde{Q}$ est le stabilisateur du drapeau

$$
\begin{aligned}
0=\mathrm{F}_{I_{0}} \subseteq \mathrm{F}_{I_{1}} \subsetneq \cdots \subsetneq \mathrm{F}_{I_{i}}=\mathrm{F}_{J_{1}} \subsetneq \mathrm{F}_{I \backslash J_{2}} \oplus D_{0}=\mathrm{F}_{\left(I \backslash J_{2}\right) \cup I_{0}^{\prime}} \oplus D_{0} \subsetneq \\
\mathrm{F}_{\left(I \backslash J_{2}\right) \cup I_{1}^{\prime}} \oplus D_{0} \subsetneq \cdots \subsetneq \mathrm{F}_{\left(I \backslash J_{2}\right) \cup I_{k-i}^{\prime}} \oplus D_{0}=\mathrm{F}_{I} \oplus D_{0} .
\end{aligned}
$$

Si l'on pose alors $j_{1}=\# J_{1}, j_{2}=\# J_{2}$ et

$$
a_{i}^{m}=\#\left\{\left(I_{0}, I_{1}, \ldots, I_{i}\right) \mid \varnothing=I_{0} \subsetneq I_{1} \subsetneq \ldots \subsetneq I_{i}=\{1,2, \ldots, m\}\right\}, \quad i, m \in \mathbb{N} .
$$

On voit que le nombre des $\widetilde{Q} \in \mathcal{F}\left(M_{\widetilde{I}_{0}}\right)$ tels que $\mathcal{I}_{\widetilde{Q}}=\mathcal{J}$ et $k=d_{\widetilde{Q}}^{\widetilde{G}}$ égale

$$
c_{k}^{j_{1}, j_{2}}:=\sum_{i=0}^{k} a_{i}^{j_{1}} a_{k-i}^{j_{2}} .
$$

On veut alors montrer que l'expression

$$
\sum_{k=0}^{j_{1}+j_{2}}(-1)^{k} c_{k}^{j_{1}, j_{2}}=\left(\sum_{i=0}^{j_{1}}(-1)^{i} a_{i}^{j_{1}}\right)\left(\sum_{j=0}^{j_{2}}(-1)^{j} a_{j}^{j_{2}}\right)
$$

égale $(-1)^{j_{1}+j_{2}}$. Or, comme il est expliqué dans le lemme 5.6 de [Zyd15a], on a pour tout $m \in \mathbb{N}$ que $\sum_{i=0}^{m}(-1)^{i} a_{i}^{m}=(-1)^{m}$ donc, le résultat suit de l'identité ci-dessus.

On vient d'obtenir alors

$$
i_{\mathfrak{o}}(x)=\sum_{\mathcal{I} \sqcup \mathcal{J} \subseteq_{\epsilon} I_{0}}(-1)^{\# \mathcal{J}} \sum_{\delta \in T_{I_{0} \backslash|\mathcal{I}|}(\mathrm{F}) \backslash G(\mathrm{~F})} \hat{f}_{\delta x}^{\mathcal{J}}\left(X_{\mathcal{I}}\right) .
$$

Lemme 5.7 (cf. corollaire 5.23). Soient $\mathcal{J}, \mathcal{J}_{1}, \mathcal{J}_{2} \subseteq_{\epsilon} I_{0}$ tels que $\mathcal{J}_{1} \sqcup \mathcal{J}_{2} \subseteq \mathcal{J}$. L'intégrale suivante

$$
\int_{T_{I_{0} \backslash\left|\mathcal{J}_{12}\right|}(\mathrm{F}) \backslash G(\mathbb{A})} \mathbb{1}_{\left(\mathcal{J} \backslash \mathcal{J}_{2}\right) \cup \mathcal{J}_{2}^{\sharp}}\left(H_{I}(x)\right) \hat{f}_{x}^{\mathcal{J} \backslash \mathcal{J}_{1}}\left(X_{\mathcal{J}_{1} \cup \mathcal{J}_{2}^{\sharp}}\right) \eta_{s}(\operatorname{det} x) d x, \quad s \in \mathbb{C}
$$

converge absolument et uniformément sur tous les compacts de $-1<\operatorname{Re}(s)<1$ et admet un prolongement méromorphe à $\mathbb{C}$, noté $\bar{\Lambda}_{\mathcal{J}_{1}, \mathcal{J}_{2}}^{\mathcal{J}}(\eta, f)(s)$, holomorphe sur $s \in \mathbb{C} \backslash\{-1,1\}$.

Le rapport entre les $\bar{\Lambda}_{\mathcal{J}_{1}, \mathcal{J}_{2}}^{\mathcal{J}}(\eta, f)(s)$ et $I_{\mathfrak{o}}\left(\eta_{s}, f\right)$ est donné par le lemme suivant.

Lemme 5.8. Pour tout $s \in \mathbb{C} \backslash\{-1,1\}$ on $a$ :

$$
I_{\mathfrak{o}}\left(\eta_{s}, f\right)=\sum_{|\mathcal{J}|=I_{0}} \sum_{\mathcal{J}_{1} \sqcup \mathcal{J}_{2} \subseteq \mathcal{J}}(-1)^{\#\left(\mathcal{J} \backslash \mathcal{J}_{12}\right)} \bar{\Lambda}_{\mathcal{J}_{1}, \mathcal{J}_{2}}^{\mathcal{J}}(\eta, f)(s) .
$$

Démonstration. En raisonnant comme dans la preuve du lemme 5.9 de [Zyd15a, on obtient pour tout $x \in G(\mathbb{A})$ :

$$
\sum_{|\mathcal{J}|=I_{0}} \sum_{\mathcal{J}_{1} \sqcup \mathcal{J}_{2} \subseteq \mathcal{J}}(-1)^{\#\left(\mathcal{J} \backslash \mathcal{J}_{12}\right)} \sum_{\delta \in T_{\left|\mathcal{J}_{12}\right|}(\mathrm{F})} \hat{f}_{\delta x}^{\mathcal{J} \backslash \mathcal{J}_{1}}\left(X_{\mathcal{J}_{1} \cup \mathcal{J}_{2}^{\sharp}}\right) \mathbb{1}_{\left(\mathcal{J} \backslash \mathcal{J}_{2}\right) \cup \mathcal{J}_{2}^{\sharp}}\left(H_{I}(x)\right)=\sum_{\mathcal{I} \sqcup \mathcal{J} \subseteq \epsilon I_{0}}(-1)^{\# \mathcal{J}} \sum_{\delta \in T_{|\mathcal{I}|}(\mathrm{F})} \hat{f}_{\delta x}^{\mathcal{J}}\left(X_{\mathcal{I}}\right) .
$$

On multiplie cette égalité par $\eta_{s}(\operatorname{det} x)$, où $s \in \mathbb{C}$ est tel que $-1<\operatorname{Re}(s)<1$. En regardant le côté droit de cette égalité et en utilisant la formule (15.11), on voit que, en vertu de la proposition 5.5. l'intégrale de cette expression sur $T_{I_{0}}(\mathrm{~F}) \backslash G(\mathbb{A})$ égale $I_{\mathfrak{o}}\left(\eta_{s}, f\right)$. Or, en vertu du corollaire 5.7 l'intégrale du côté gauche sur le même quotient donne l'égalité cherchée pour $s \in \mathbb{C}$ tels que $-1<\operatorname{Re}(s)<1$. Ceci suffit pour conclure car $s \mapsto I_{\mathfrak{o}}\left(\eta_{s}, f\right)$ est une fonction méromorphe en vertu de la remarque 3.10 . 
On introduit maintenant les fonctions zêta.

Proposition 5.9 (cf. 5.20 et lemme 5.22). Soit $\mathcal{J} \subseteq_{\epsilon} I_{0}$. Alors l'intégrale

$$
\int_{G\left(\mathbb{A}, X_{\mathcal{J}}\right) \backslash G(\mathbb{A})} f\left(x^{-1} X_{\mathcal{J}} x\right) e^{\lambda\left(H_{I}(x)\right)} \eta(\operatorname{det} x) d x, \quad \lambda \in \mathfrak{a}_{|\mathcal{J}|, \mathbb{C}}^{*}
$$

converge absolument et uniformément sur tous les compacts d'un ouvert non-vide de $\mathfrak{a}_{|\mathcal{J}|, \mathbb{C}}^{*}$ et admet un prolongement méromorphe à $\mathfrak{a}_{|\mathcal{J}|, \mathbb{C}}^{*}$, noté $\zeta_{\mathcal{J}}(\eta, f)$. En plus, la droite $\mathbb{C} \operatorname{det} \subseteq \mathfrak{a}_{|\mathcal{J}|, \mathbb{C}}^{*}$ est non-singulière.

Corollaire 5.10. Pour $\mathcal{J} \subseteq_{\epsilon} I_{0}$ on définit la fonction méromorphe sur $\mathbb{C}$ suivante:

$$
\zeta_{\mathcal{J}}(\eta, f)(s):=\zeta_{\mathcal{J}}(\eta, f)(s \operatorname{det}), \quad s \in \mathbb{C}
$$

où l'on voit det comme un élément de $\mathfrak{a}_{|\mathcal{J}|}^{*}$ par restriction.

Remarque 5.11. Il découle de la proposition 5.20, que pour certains $\mathcal{J} \subseteq_{\epsilon} I_{0}$, pour tout $s \in \mathbb{C}$ l'élément $s$ det $\in \mathfrak{a}_{|\mathcal{J}|, \mathbb{C}}^{*}$ n'appartient pas au domaine de convergence de l'intégrale définissant $\zeta_{\mathcal{J}}(\eta, f)$.

Soit $I_{\eta} \subseteq I_{0}$ l'ensemble de $i \in I_{0}$ tels que la restriction de $\eta$ au groupe de normes de $\mathbb{A}_{i}^{*}$ dans $\mathbb{A}^{*}$ est non-triviale. On est prêt à démontrer le résultat principal de cette section.

Théoréme 5.12. Soit $f \in \mathcal{S}(\widetilde{\mathfrak{g}}(\mathbb{A}))$.

i) Pour tout $\mathcal{J}_{1} \subseteq_{\epsilon} I_{\eta}$ tel que $\left|\mathcal{J}_{1}\right|=I_{\eta}$ la fonction de la variable $s \in \mathbb{C}$ suivante

$$
\sum_{\left|\mathcal{J}^{\prime}\right|=I_{0} \backslash I_{\eta}} \zeta_{\mathcal{J}_{1} \cup \mathcal{J}^{\prime}}(\eta, f)
$$

est holomorphe sur $\mathbb{C} \backslash\{-1,1\}$.

ii) On a pour tout $s \in \mathbb{C} \backslash\{-1,1\}$ :

$$
I_{\mathfrak{o}}\left(\eta_{s}, f\right)=\sum_{\left|\mathcal{J}_{1}\right|=I_{\eta}}\left(\left(\sum_{\left|\mathcal{J}^{\prime}\right|=I_{0} \backslash I_{\eta}} \zeta_{\mathcal{J}_{\eta} \cup \mathcal{J}^{\prime}}(\eta, f)\right)(s)\right) .
$$

Démonstration. Le point i) est démontré dans le corollaire 5.24 ci-dessous. En outre, en vertu du lemme 5.27 de [Zyd15a], on a l'égalité suivante des fonctions méromorphes sur $\mathbb{C}$ :

$$
\sum_{|\mathcal{J}|=I_{0}} \sum_{\mathcal{J}_{1} \sqcup \mathcal{J}_{2} \subseteq \mathcal{J}}(-1)^{\#\left(\mathcal{J} \backslash \mathcal{J}_{12}\right)} \bar{\Lambda}_{\mathcal{J}_{1}, \mathcal{J}_{2}}^{\mathcal{J}}(\eta, f)=\sum_{|\mathcal{J}|=I_{0}} \zeta_{\mathcal{J}}(\eta, f) .
$$

En utilisant alors l'égalité démontrée dans le lemme 5.8 ainsi que le point i) de ce théorème on peut conclure.

\subsection{Deuxième formule pour le noyau tronqué}

On considère $f \in \mathcal{S}(\widetilde{\mathfrak{g}}(\mathbb{A}))$ fixée. Pour $\widetilde{P} \in \mathcal{F}\left(M_{\widetilde{0}}, B\right)$, dans le paragraphe 5.4, équation (5.6), on a introduit la fonction $f^{\widetilde{P}}$ ainsi que $f_{x}(X):=f\left(\operatorname{Ad}\left(x^{-1}\right) X\right)$. Par $f_{x}^{\widetilde{P}}$ on entend toujours $\left(f_{x}\right)^{\widetilde{P}}$. Soient $\widetilde{P}, \widetilde{Q} \in \mathcal{F}\left(M_{\widetilde{0}}, B\right)$ tels que $\widetilde{P} \subseteq \widetilde{Q}$. On pose alors

$$
\mathcal{V}_{\widetilde{P}}^{\widetilde{Q}}:=\mathcal{V}_{\widetilde{P}} \cap \mathcal{Z}_{\widetilde{Q}}
$$


Alors, pour $\widetilde{Q}$ fixé, les espaces $\mathcal{V}_{\widetilde{P}}^{\widetilde{Q}}$, où $\mathcal{F}\left(M_{\widetilde{0}}, B\right) \ni \widetilde{P} \subseteq \widetilde{Q}$, jouent le rôle des espaces $\mathcal{V}_{\widetilde{P}}$ dans le contexte de l'inclusion $G_{\widetilde{Q}} \hookrightarrow \widetilde{G}_{\widetilde{Q}}$. En plus, on a les relations suivantes :

$$
\mathfrak{n}_{Q} \oplus \mathcal{V}_{\widetilde{P}}=\mathfrak{n}_{\widetilde{Q}} \oplus \mathcal{V}_{\widetilde{P}}^{\widetilde{Q}}, \quad \mathfrak{n}_{\widetilde{P}}^{\widetilde{Q}} \oplus \mathfrak{n}_{Q}=\mathfrak{n}_{P} \oplus \mathcal{V}_{\widetilde{P}}^{\widetilde{Q}}
$$

Les lemmes suivants sont des analogues des corollaires 5.13 et 5.14 de Zyd15a].

Lemme 5.13. Soient $\widetilde{P} \in \mathcal{F}\left(M_{\widetilde{0}}, B\right), \xi \in \mathfrak{m}_{\widetilde{P}}(\mathrm{~F}) \cap \mathfrak{o}$ et $x \in G(\mathbb{A})$, alors

$$
\sum_{\gamma \in N_{P}(\mathrm{~F})} f_{\gamma x}^{\widetilde{P}}(\xi)=\sum_{\zeta \in \mathfrak{n}_{P}(\mathrm{~F})} f_{x}^{\widetilde{P}}(\xi+\zeta)
$$

Lemme 5.14. Soient $\widetilde{P} \in \mathcal{F}\left(M_{\widetilde{0}}, B\right), \xi \in \mathfrak{m}_{\widetilde{P}}(\mathrm{~F}) \cap \mathfrak{o}$ et $x \in G(\mathbb{A})$, alors

$$
\int_{N_{P}(\mathbb{A})} f_{n x}^{\widetilde{P}}(\xi) d n=\int_{\mathfrak{n}_{\widetilde{P}}(\mathbb{A})} f_{x}(\xi+U) d U
$$

Pour $T \in \mathfrak{a}_{\tilde{0}}^{+}$posons

$$
i_{\mathfrak{o}}^{T}(x)=i_{f, \mathfrak{o}}^{T}(x)=\sum_{\widetilde{P} \in \mathcal{F}\left(M_{\widetilde{0}}, B\right)}(-1)^{d d_{\widetilde{P}}^{\widetilde{T}}} \sum_{\delta \in P(\mathrm{~F}) \backslash G(\mathrm{~F})} \hat{\tau}_{\widetilde{P}}\left(H_{\widetilde{P}}(\delta x)-T_{\widetilde{P}}\right) i_{\widetilde{P}, \mathfrak{o}}(\delta x), x \in G(\mathrm{~F}) \backslash G(\mathbb{A})
$$

où $i_{\widetilde{P}, \mathfrak{o}}$ est définie dans la section 5.4 par (5.7). La fonction $i_{\mathfrak{o}}^{T}$ est une variante de $k_{\mathfrak{o}}^{T}$ définie au début du paragraphe 2.2 .

Théoréme 5.15. Pour tous $T \in T_{+}+\mathfrak{a}_{\widetilde{0}}^{+}, f \in \mathcal{S}(\widetilde{\mathfrak{g}}(\mathbb{A})), \sigma \in \mathbb{R}$ et $\mathfrak{o} \in \mathcal{O}_{\text {rs }}$, on a :

$$
\int_{G(\mathrm{~F}) \backslash G(\mathbb{A})}\left|i_{f, \mathfrak{o}}^{T}(x)\right||\operatorname{det} x|_{\mathbb{A}}^{\sigma} d x<\infty .
$$

Démonstration. En procédant comme au début de la preuve du théorème 2.6 on montre que l'intégrale $\int_{[G]}\left|i_{f, \mathfrak{o}}^{T}(x) \| \operatorname{det} x\right|_{\mathbb{A}}^{\sigma} d x$ est majorée par la somme sur les sous-groupes paraboliques relativement standards $\widetilde{P}_{1} \subseteq \widetilde{S} \subseteq \widetilde{P}_{2}$ de

$$
\int_{P_{1}(\mathrm{~F}) \backslash G(\mathbb{A})} \sum_{\xi \in\left(\mathfrak{m} \widetilde{\mathfrak{S}}_{1}^{\widetilde{S}}\right)^{\prime}(\mathrm{F}) \cap \mathfrak{o}} \chi_{\widetilde{1}, \widetilde{2}}^{T}(x)\left|\sum_{\widetilde{S} \subseteq \widetilde{P} \subseteq \widetilde{P}_{2}}(-1)^{d d_{\widetilde{P}}^{\widetilde{G}}} \sum_{\zeta \in \mathfrak{n}_{\widetilde{S}}^{\widetilde{P}}(\mathrm{~F})} \sum_{\gamma \in N_{P}(\mathrm{~F})} f_{\gamma x}^{\widetilde{P}}(\xi+\zeta) \| \operatorname{det} x\right|_{\mathbb{A}}^{\sigma} d x .
$$

En utilisant le lemme 5.13 ci-dessus (pour la fonction $X \mapsto f(X+V)$ où $V \in \mathfrak{n} \underset{\widetilde{S}}{\widetilde{P}}(\mathrm{~F})$ ) et ensuite la formule sommatoire de Poisson on s'aperçoit que la somme entre la valeur absolue dans l'intégrale ci-dessus égale

$$
\left|\sum_{\widetilde{S} \subseteq \widetilde{P} \subseteq \widetilde{P}_{2}}(-1)^{d d_{\widetilde{P}}^{\widetilde{\widetilde{P}}}} \sum_{\zeta_{1} \in \tilde{\mathfrak{n}}_{\widetilde{S}}(\mathrm{~F})} \sum_{\zeta_{2} \in \overline{\mathfrak{n}}_{P}(\mathrm{~F})} \widetilde{\phi}_{\widetilde{S}}\left(x, \xi, \zeta_{1}+\zeta_{2}\right)\right|
$$

où :

$$
\widetilde{\phi}_{\widetilde{S}}(x, X, Y)=\int_{\mathfrak{n}_{\widetilde{S}}(\mathbb{A})} f\left(\operatorname{Ad}\left(x^{-1}\right)(X+U)\right) \Psi(\langle U, Y\rangle) d U, x \in G(\mathbb{A}), X \in \mathfrak{m}_{\widetilde{P}_{1}}^{\widetilde{S}}(\mathbb{A}), Y \in \overline{\mathfrak{n}}_{\widetilde{S}}(\mathbb{A})
$$

et $\overline{\mathfrak{n}}_{P}$ est le radical nilpotent de l'algèbre de Lie du sous-groupe parabolique opposé à $P$. 
On pose $\mathfrak{l}_{\widetilde{S}}^{\widetilde{P}}=\overline{\mathfrak{n}}_{\widetilde{S}}^{\widetilde{P}} \oplus \overline{\mathfrak{n}}_{P}$. Grâce aux décompositions $(\underline{(5.13)})$ on voit qu'on a $\mathfrak{l}_{\widetilde{S}}^{\widetilde{R}} \subseteq \mathfrak{l} \mathfrak{l}_{\widetilde{S}}^{\widetilde{P}}$ pour tout $\widetilde{S} \subseteq \widetilde{R} \subseteq \widetilde{P}$. On pose donc

$$
(\mathfrak{l} \widetilde{P})^{\prime}=\mathfrak{l} \mathfrak{l}_{\widetilde{S}}^{\widetilde{P}} \backslash \underset{\widetilde{S} \subseteq \widetilde{R} \subsetneq \widetilde{P}}{\bigcup} \mathfrak{l} \widetilde{S}
$$

On a donc $\mathfrak{l}_{\widetilde{S}}^{\widetilde{P}}=\coprod_{\widetilde{S} \subseteq \widetilde{R} \subseteq \widetilde{P}}\left(\mathfrak{l}_{\widetilde{S}}^{\widetilde{R}}\right)^{\prime}$ et par un raisonnement habituel basé sur l'identité (2.10) on s'aperçoit que (5.14) égale

$$
\left|\sum_{\zeta \in\left(\widetilde{l}_{\widetilde{S}}^{2}\right)^{\prime}(\mathrm{F})} \widetilde{\phi}_{\widetilde{S}}(x, \xi, \zeta)\right|
$$

Posons alors

$$
\Psi_{\widetilde{S}}(x, X, Y)=\sum_{\zeta_{2} \in \overline{\mathfrak{n}}_{2}(\mathrm{~F})} \widetilde{\phi}_{\widetilde{S}}\left(x, X, Y+\zeta_{2}\right), \quad x \in G(\mathbb{A}), X \in \mathfrak{m}_{\widetilde{1}}^{\widetilde{S}}(\mathbb{A}), Y \in \overline{\mathfrak{n}}_{\widetilde{S}}^{\widetilde{2}}(\mathbb{A}) .
$$

Alors $\Psi_{S}(x, X, Y) \in \mathcal{S}\left(\left(\mathfrak{m}_{\widetilde{1}}^{\widetilde{S}} \oplus \overline{\mathfrak{n}}_{\widetilde{S}}^{\widetilde{2}}\right)(\mathbb{A})\right)$ pour un $x$ fixé et on a :

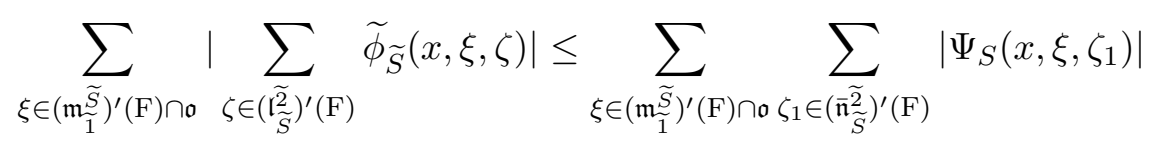

car l'image de $\left(\widetilde{l}_{\widetilde{S}}^{\widetilde{2}}\right)^{\prime}$ par la projection naturelle sur $\widetilde{\mathfrak{n}}_{\widetilde{S}}^{\widetilde{2}}$ est contenue dans $\left(\overline{\mathfrak{n}}_{\widetilde{S}}^{\widetilde{2}}\right)^{\prime}$.

On se ramène alors à borner, pour $\widetilde{P}_{1} \subseteq \widetilde{S} \subseteq \widetilde{P}_{2}$ fixés :

$$
\int_{P_{1}(\mathrm{~F}) \backslash G(\mathbb{A})} \chi_{\widetilde{1}, \widetilde{2}}^{T}(x) \sum_{\xi \in\left(\mathfrak{m} \widetilde{\mathfrak{T}}_{1}\right)^{\prime}(\mathrm{F}) \cap \mathfrak{o}} \sum_{\zeta_{1} \in\left(\tilde{\mathfrak{n}}_{\widetilde{S}}^{2}\right)^{\prime}(\mathrm{F})}\left|\Phi_{\widetilde{S}}\left(x, \xi, \zeta_{1}\right)\right||\operatorname{det} x|_{\mathbb{A}}^{\sigma} d x .
$$

Cette intégrale est identique à (2.11) du théorème 2.6. Cela conclut la preuve.

Proposition 5.16. Pour $T \in T_{+}+\mathfrak{a}_{\tilde{0}}^{+}, s \in \mathbb{C}, f \in \mathcal{S}(\widetilde{\mathfrak{g}}(\mathbb{A}))$ et $\mathfrak{o} \in \mathcal{O}_{r s}$, on a :

$$
I_{\mathfrak{o}}^{T}\left(\eta_{s}, f\right)=\int_{G(\mathrm{~F}) \backslash G(\mathbb{A})} i_{f, \mathfrak{o}}^{T}(x) \eta_{s}(\operatorname{det} x) d x .
$$

Démonstration. Dans la preuve on utilisera la notation du paragraphe 2.2 introduite au début de la preuve du théorème 2.6. Donc, en raisonnant comme au début de la preuve de ce théorème-là, on voit que

$$
\int_{[G]} i_{f, \mathfrak{o}}^{T}(x) \eta_{s}(\operatorname{det} x) d x=\sum_{\widetilde{P}_{1} \in \mathcal{F}\left(M_{\widetilde{0}}, B\right)} \sum_{\widetilde{P}_{2} \supseteq \widetilde{P}_{1}} \int_{P_{1}(\mathrm{~F}) \backslash G(\mathbb{A})} \chi_{\widetilde{1}, \widetilde{2}}^{T}(x) \sum_{\widetilde{P}_{1} \subseteq \widetilde{P} \subseteq \widetilde{P}_{2}}(-1)^{d \widetilde{P}_{\tilde{P}}^{\widetilde{\widetilde{P}}}} i_{\widetilde{P}, \mathfrak{o}}(x) \eta_{s}(\operatorname{det} x) d x .
$$

Fixons les sous-groupes paraboliques relativement standards $\widetilde{P}_{1} \subseteq \widetilde{P}_{2}$ et décomposons l'intégrale sur $P_{1}(\mathrm{~F}) \backslash G(\mathbb{A})$ en une double intégrale sur $x \in M_{1}(\mathrm{~F}) N_{1}(\mathbb{A}) \backslash G(\mathbb{A})$ et $n_{1} \in N_{1}(\mathrm{~F}) \backslash N_{1}(\mathbb{A})$. Ensuite on fait passer cette dernière intégrale à l'intérieure de la somme sur $\widetilde{P}$. On peut le faire car pour tout $\widetilde{P}_{1} \subseteq \widetilde{P} \subseteq \widetilde{P}_{2}$ la fonction $N_{1}(\mathbb{A}) \ni n_{1} \mapsto i_{\mathfrak{o}, \widetilde{P}}\left(n_{1} x\right)$ est $N_{1}(\mathrm{~F})$-invariante et continue. Comme le volume de $N_{P}(\mathrm{~F}) \backslash N_{P}(\mathbb{A})$ vaut 1 et $N_{P} \subseteq N_{1}$ on a

$$
\int_{\left[N_{1}\right]} i_{\widetilde{P}, \mathfrak{o}}\left(n_{1} x\right) d n_{1}=\int_{\left[N_{1}\right]} \sum_{\xi \in \mathfrak{m}_{\widetilde{P}}(\mathrm{~F}) \cap_{[}} \int_{\left[N_{P}\right]} \sum_{\gamma \in N_{P}(\mathrm{~F})} f_{\gamma n n_{1} x}^{\widetilde{P}}(\xi) d n d n_{1}=\int_{\left[N_{1}\right]} \sum_{\xi \in \mathfrak{m}_{\widetilde{P}}(\mathrm{~F}) \cap \mathfrak{o}} \int_{N_{P}(\mathbb{A})} f_{n n_{1} x}^{\widetilde{P}}(\xi) d n d n_{1} .
$$


La dernière expression égale $\int_{\left[N_{1}\right]} k_{\widetilde{P}, \mathfrak{o}}\left(n_{1} x\right) d n_{1}$ en vertu du lemme 5.14, On intervertit de nouveau la somme qui porte sur $\widetilde{P}$ avec l'intégrale sur $N_{1}(\mathrm{~F}) \backslash N_{1}(\mathbb{A})$ et l'on recombine cette dernière avec l'intégrale sur $M_{1}(\mathrm{~F}) N_{1}(\mathbb{A}) \backslash G(\mathbb{A})$. On retrouve donc

$$
\int_{G(\mathrm{~F}) \backslash G(\mathbb{A})} i_{f, \mathfrak{o}}^{T}(x) \eta_{s}(\operatorname{det} x) d x=\sum_{\widetilde{P}_{1} \in \mathcal{F}\left(M_{\widetilde{0}}, B\right)} \sum_{\widetilde{P}_{2} \supseteq \widetilde{P}_{1}} \int_{P_{1}(\mathrm{~F}) \backslash G(\mathbb{A})} \chi_{\widetilde{1}, \widetilde{2}}^{T}(x) k_{\widetilde{1}, \widetilde{2}, \mathfrak{o}}(x) \eta_{s}(\operatorname{det} x) d x .
$$

Chaque intégrale dans la somme ci-dessus converge d'après le théorème 2.7 ce qui justifie l'intégration et de surcroît, la somme elle-même égale $I_{\mathrm{o}}^{T}\left(\eta_{s}, f\right)$ grâce à l'identité (2.6) et la définition de $I_{\mathfrak{o}}^{T}\left(\eta_{s}, f\right)$ donnée au début de la section 3, ce qu'il fallait démontrer.

Plaçons-nous maintenant dans le cadre du paragraphe 3.2. On veut généraliser le théorème 5.15 et la proposition 5.16 au cas de 1 inclusion $H \times G^{\prime} \hookrightarrow H \times \widetilde{G}^{\prime}$.

Notons $\mathcal{O}_{r s}^{H \times \widetilde{G}^{\prime}}$ l'ensemble de classes contenant un élément $X_{1}+X_{2} \in \mathfrak{h}(\mathrm{F}) \times \widetilde{\mathfrak{g}}^{\prime}(\mathrm{F})$ tel que le polynôme caractéristique de $X_{1}$ est séparable et tel que $X_{2} \in \widetilde{\mathfrak{g}}^{\prime}(\mathrm{F})$ appartient à une classe relativement semi-simple régulière dans le contexte d'inclusion $G^{\prime} \hookrightarrow \widetilde{G}^{\prime}$.

Pour $f \in \mathcal{S}\left(\left(\mathfrak{h} \times \widetilde{\mathfrak{g}}^{\prime}\right)(\mathbb{A})\right), \mathfrak{o} \in \mathcal{O}_{r s}^{H \times \widetilde{G}^{\prime}}$ et $\widetilde{P} \in \mathcal{F}_{H \times G^{\prime}}\left(M_{\widetilde{0}}, B\right)$ soit

$$
i_{f, \widetilde{P}, \mathfrak{o}}(x)=\sum_{\xi \in \mathfrak{m}_{\widetilde{P}}(\mathrm{~F}) \cap \mathfrak{o}} \sum_{\gamma \in N_{P}(\mathrm{~F})} \int_{\mathcal{V}_{\widetilde{P}}^{\prime}(\mathbb{A})} f\left(\operatorname{Ad}\left((\gamma x)^{-1}\right)\left(\xi+Y_{\widetilde{P}}^{\prime}\right)\right) d Y_{\widetilde{P}}^{\prime}
$$

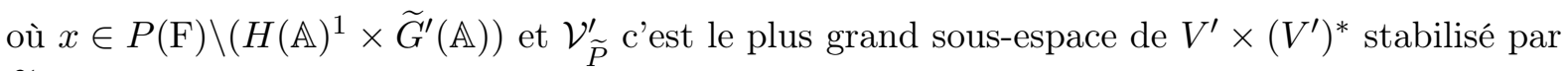
$\widetilde{P}$.

Pour $T \in \mathfrak{a}_{\widetilde{B}_{0}}^{+}$on pose aussi

$$
i_{f, \mathfrak{o}}^{T}(x)=\sum_{\widetilde{P} \in \mathcal{F}_{H \times G^{\prime}}\left(M_{\widetilde{0}}, B\right)}(-1)^{d_{\widetilde{P}}^{H} \times \widetilde{G}^{\prime}} \sum_{\delta \in P(\mathrm{~F}) \backslash\left(H \times G^{\prime}\right)(\mathrm{F})} \hat{\tau}_{\widetilde{P} \times \widetilde{G}^{\prime}}^{H}\left(H_{\widetilde{P}}(\delta x)-T_{\widetilde{P}}\right) i_{f, \widetilde{P}, \mathfrak{o}}(\delta x), \quad x \in\left(H \times G^{\prime}\right)(\mathrm{F}) \backslash\left(H(\mathbb{A})^{1} \times G^{\prime}(\mathbb{A})\right) .
$$

La preuve du théorème 5.15 s'étend sans problème dans ce cas donnant pour tout $\sigma \in \mathbb{R}$

$$
\int_{\left(H \times G^{\prime}\right)(\mathrm{F}) \backslash\left(H(\mathbb{A})^{1} \times G^{\prime}(\mathbb{A})\right)}\left|i_{f, \mathfrak{o}}^{T}(x)\right||\operatorname{det} x|_{\mathbb{A}}^{\sigma} d x<\infty
$$

pour $T$ suffisamment régulier. De même la preuve de la proposition 5.16 s'étend aussi bien et l'on obtient, avec la notation du paragraphe 3.2, pour $\mathfrak{o} \in \mathcal{O}_{r s}^{H \times \widetilde{G}^{\prime}}$ et $s \in \mathbb{C}$

$$
\int_{\left(H \times G^{\prime}\right)(\mathrm{F}) \backslash\left(H(\mathbb{A})^{1} \times G^{\prime}(\mathbb{A})\right)} i_{f, \mathfrak{o}}^{T}(x) \eta_{s}(\operatorname{det} x) d x=I_{\mathfrak{o}}^{H \times \widetilde{G}^{\prime}, T}\left(\eta_{s}, f\right) .
$$

Soient maintenant $\widetilde{Q} \in \mathcal{F}\left(M_{\widetilde{0}}, B\right), \mathfrak{o} \in \mathcal{O}_{r s}$ et $\mathfrak{o}_{\widetilde{Q}, 1}, \ldots, \mathfrak{o}_{\widetilde{Q}, m} \in \mathcal{O}^{M_{\widetilde{Q}}}$ comme dans l'équation (3.5). Alors $\mathfrak{o}_{\widetilde{Q}, i} \in \mathcal{O}_{r s}^{M_{\widetilde{Q}}}$ pour $i=1, \ldots, m$. On a dans ce cas l'analogue suivant de l'égalité (3.8) : pour tout sous-groupe de Borel relativement standard $\widetilde{B} \subseteq \widetilde{Q}$ et tous $f \in \mathcal{S}(\widetilde{\mathfrak{g}}(\mathbb{A}))$ et $s \in \mathbb{C}$

$$
\begin{aligned}
& I_{I_{\mathfrak{o}}}^{M_{\widetilde{Q}}, T}\left(\eta_{s}, f_{\widetilde{Q}}\right)=\int_{M_{Q}(\mathrm{~F}) \backslash H_{Q}(\mathbb{A})^{1} \times G_{\widetilde{Q}}(\mathbb{A})} \sum_{i=1}^{m} i_{f_{\widetilde{Q}}, \mathfrak{o}_{\widetilde{Q}, i}}^{T_{\widetilde{Q}}}(m) \eta_{s_{\widetilde{Q}}}(\operatorname{det} m) d m=\int_{M_{Q}(\mathrm{~F}) \backslash H_{Q}(\mathbb{A})^{1} \times G_{\widetilde{Q}}(\mathbb{A})} e^{-\rho_{\widetilde{Q}, s}\left(H_{\widetilde{Q}}(m)\right)} \sum_{\widetilde{P} \subseteq \widetilde{Q}}(-1)^{d_{\widetilde{P}}^{\widetilde{P}}} \\
& \sum_{\delta \in\left(P \cap M_{Q}\right)(\mathrm{F}) \backslash M_{Q}(\mathrm{~F})} \hat{\tau}_{P}^{Q}\left(H_{P}(\delta m)-T\right)\left(\sum_{\substack{\xi \in \mathfrak{m}_{\widetilde{P}}(\mathrm{~F}) \cap \mathfrak{0} \\
\gamma \in N_{P}^{Q}(\mathrm{~F})}} \int_{\substack{\widetilde{Q} \\
\mathcal{V}_{\widetilde{P}}(\mathbb{A})}} f_{\widetilde{Q}}\left(\operatorname{Ad}\left((\gamma \delta m)^{-1}\right)(\xi+Y)\right) d Y\right) \eta_{s}(\operatorname{det} m) d m .
\end{aligned}
$$




\subsection{Expression intégrale de $J_{\mathfrak{o}}$}

Dans ce paragraphe, on démontre la proposition suivante, énoncée dans le paragraphe 5.4 par la proposition 5.5 .

Proposition 5.17. Pour tout $s \in \mathbb{C}$ tel que $-1<\operatorname{Re}(s)<1$ on a

$$
\int_{G(\mathrm{~F}) \backslash G(\mathbb{A})}\left|i_{f, \mathfrak{o}}(x) \eta_{s}(\operatorname{det} x)\right| d x<\infty \quad \text { et } \quad \int_{G(\mathrm{~F}) \backslash G(\mathbb{A})} i_{f, \mathfrak{o}}(x) \eta_{s}(\operatorname{det} x) d x=I_{\mathfrak{o}}\left(\eta_{s}, f\right) .
$$

Démonstration. Pour tout $\widetilde{Q} \in \mathcal{F}\left(M_{\widetilde{0}}, B\right)$ soit $\bar{\tau}_{\widetilde{Q}}$ la fonction caractéristique de $H \in \mathfrak{a}_{\widetilde{Q}}$ tels que $\alpha(H) \leq 0$ pour tout $\alpha \in \Delta_{\widetilde{Q}}$. Il résulte du lemme combinatoire de Langlands (Proposition 1.7.2 de [LW09]) que pour tout $\widetilde{P} \in \mathcal{F}\left(M_{\widetilde{0}}, B\right)$ et tout $H \in \mathfrak{a}_{\widetilde{P}}$ on a

$$
\sum_{\widetilde{Q} \supseteq \widetilde{P}} \hat{\tau}_{\widetilde{P}}^{\widetilde{Q}}(H) \bar{\tau}_{\widetilde{Q}}(H)=1
$$

En utilisant cette identité, on a pour tout $T \in T_{+}+\mathfrak{a}_{\tilde{0}}^{+}$

$$
\begin{aligned}
& i_{f, \mathfrak{o}}(x)=\sum_{\widetilde{P} \in \mathcal{F}\left(M_{\widetilde{0}}, B\right)}(-1)^{d d_{\widetilde{P}}^{\widetilde{G}}} \sum_{\gamma \in P(\mathrm{~F}) \backslash G(\mathrm{~F})} i_{\widetilde{P}, \mathfrak{o}}(\gamma x)=\sum_{\widetilde{P} \in \mathcal{F}\left(M_{\widetilde{0}}, B\right)}(-1)^{d \widetilde{P}_{\widetilde{P}}^{\widetilde{Q}}} \\
& \sum_{\gamma \in P(\mathrm{~F}) \backslash G(\mathrm{~F})} i_{\widetilde{P}, \mathfrak{o}}(\gamma x)\left(\sum_{\widetilde{Q} \supseteq \widetilde{P}} \hat{\tau}_{\widetilde{P}}^{\widetilde{Q}}\left(H_{\widetilde{P}}(\gamma x)-T_{\widetilde{P}}\right) \bar{\tau}_{\widetilde{Q}}\left(H_{\widetilde{Q}}(\gamma x)-T_{\widetilde{Q}}\right)\right)=\sum_{\widetilde{Q} \in \mathcal{F}\left(M_{\widetilde{0}}, B\right)}(-1)^{d d_{\widetilde{Q}}^{\widetilde{Q}}} \\
& \sum_{\gamma \in Q(\mathrm{~F}) \backslash G(\mathrm{~F})} \bar{\tau}_{Q}\left(H_{Q}(\gamma x)-T_{Q}\right) \sum_{\mathcal{F}\left(M_{\widetilde{0}}, B\right) \ni \widetilde{P} \subseteq \widetilde{Q}}(-1)^{d d_{\widetilde{P}}^{\widetilde{Q}}} \sum_{\delta \in\left(M_{Q} \cap P\right)(\mathrm{F}) \backslash M_{Q}(\mathrm{~F})} i_{\widetilde{P}, \mathfrak{p}}(\eta \gamma x) \hat{\tau}_{\widetilde{P}}{ }^{\widetilde{Q}}\left(H_{\widetilde{P}}(\delta \gamma x)-T_{\widetilde{P}}\right)
\end{aligned}
$$

où les sommes sont absolument convergentes. Il suffit de montrer que pour tout $\widetilde{Q} \in \mathcal{F}\left(M_{\widetilde{0}}, B\right)$ et tout $s \in \mathbb{C}$ tel que $-1<\operatorname{Re}(s)<1$ l'intégrale

$$
\int_{Q(\mathrm{~F}) \backslash G(\mathbb{A})} \bar{\tau}_{\widetilde{Q}}\left(H_{\widetilde{Q}}(x)-T_{\widetilde{Q}}\right) \sum_{\mathcal{F}\left(M_{\widetilde{0}}, B\right) \ni \widetilde{P} \subseteq \widetilde{Q}}(-1)^{d d_{\widetilde{Q}}^{\widetilde{P}}} \sum_{\delta \in\left(M_{Q} \cap P\right)(\mathrm{F}) \backslash M_{Q}(\mathrm{~F})} i_{\widetilde{P}, \mathfrak{P}}(\delta x) \hat{\tau}_{\widetilde{P}}^{\widetilde{Q}}\left(H_{\widetilde{P}}(\delta x)-T_{P}\right) \eta_{s}(\operatorname{det} x) d x
$$

converge absolument. L'analyse va être analogue à celle de la preuve du théorème 3.8 ,

Posons $x=n a m k$ où $n \in N_{Q}(\mathrm{~F}) \backslash N_{Q}(\mathbb{A}), m \in M_{Q}(\mathrm{~F}) \backslash H_{\widetilde{Q}}(\mathbb{A})^{1} \times G_{\widetilde{Q}}(\mathbb{A}), a \in A_{\widetilde{Q}}^{s t, \infty}$ et $k \in K$. Donc $d x=e^{-2 \rho_{Q}\left(H_{Q}(a m)\right)} d n d a d m d k$.

Fixons $\widetilde{P} \in \mathcal{F}\left(M_{\widetilde{0}}, B\right)$ tel que $\widetilde{P} \subseteq \widetilde{Q}$. Pour $n, m, k$ et $a$ comme ci-dessus et $\delta \in M_{Q}(\mathrm{~F})$ on a $\bar{\tau}_{\widetilde{Q}}\left(H_{\widetilde{Q}}(\delta n a m k)-T_{\widetilde{Q}}\right)=\bar{\tau}_{\widetilde{Q}}\left(H_{\widetilde{Q}}(m a)-T_{\widetilde{Q}}\right)$ et, en faisant les changements de variable $\delta a^{-1} n a \delta^{-1} \mapsto n$ et $a^{-1} Y_{\widetilde{P}} \mapsto Y_{\widetilde{P}}$

$$
\begin{array}{r}
\int_{\left[N_{Q}\right]} i_{\widetilde{P}, \mathfrak{o}}(\delta n a m k) \hat{\tau}_{\widetilde{P}}^{\widetilde{Q}}\left(H_{\widetilde{P}}(\delta n a m k)-T_{\widetilde{P}}\right) d n=\int_{K} \int_{\left[N_{Q}\right]} i_{\widetilde{P}, \mathfrak{o}}(\delta n a m k) \hat{\tau}_{\widetilde{P}} \widetilde{Q}\left(H_{\widetilde{P}}(\delta m)-T_{\widetilde{P}}\right) d n= \\
\hat{\tau}_{\widetilde{P}}^{\widetilde{Q}}\left(H_{\widetilde{P}}(\delta m)-T_{\widetilde{P}}\right) e^{2 \rho_{\widetilde{Q}}\left(H_{Q}(a)\right)} \sum_{\xi \in \mathfrak{m}_{\widetilde{P}}(\mathrm{~F}) \cap \mathfrak{o}} \sum_{\gamma \in N_{P}^{Q}(\mathrm{~F}) N_{Q}(\mathbb{A})} \int_{\mathcal{V}_{\widetilde{P}}(\mathbb{A})} f_{n \gamma \delta m k}(\xi+Y) d Y d n
\end{array}
$$

où l'on utilise le fait que la somme de poids pour $A_{H_{\widetilde{Q}}} \subseteq A_{\widetilde{Q}}$ agissant sur $\mathfrak{n}_{Q} \oplus \mathcal{V}_{\widetilde{P}}=\mathfrak{n}_{\widetilde{Q}} \oplus \mathcal{V}_{\widetilde{P}}^{\widetilde{Q}}$ (voir paragraphe 5.5, equations $(\underline{5.12})$ et $(\underline{5.13})$ ) égale $2 \rho_{\widetilde{Q}}$ car $A_{H_{\widetilde{Q}}}$ agit trivialement sur $\mathcal{Z}_{\widetilde{Q}} \supseteq \mathcal{V}_{\widetilde{P}}^{\widetilde{Q}}$. 
En utilisant le lemme 5.14, on a pour tout $\xi \in \mathfrak{m}_{\widetilde{P}}(\mathrm{~F}) \cap \mathfrak{o}$ fixé

$$
\int_{N_{Q}(\mathbb{A})} \int_{\mathcal{V}_{\widetilde{P}}(\mathbb{A})} f_{n \gamma \delta m k}\left(\xi+Y_{\widetilde{P}}\right) d Y_{\widetilde{P}} d n=\int_{\mathcal{V}_{\widetilde{P}}^{\widetilde{Q}}(\mathbb{A})} \int_{\mathfrak{n}_{\widetilde{Q}}(\mathbb{A})} f_{\gamma \delta m k}\left(\xi+U_{\widetilde{Q}}+Y_{\widetilde{P}}^{\widetilde{Q}}\right) d U_{\widetilde{Q}} d Y_{\widetilde{P}}^{\widetilde{Q}}
$$

On voit alors que l'intégrale sur $k \in K$ de (5.17) fois $\eta(\operatorname{det} k)$ devient :

$$
e^{2 \rho_{\widetilde{Q}}\left(H_{Q}(a)\right)} \hat{\tau}_{\widetilde{P}}^{\widetilde{Q}}\left(H_{\widetilde{P}}(\delta m)-T_{\widetilde{P}}\right) \sum_{\xi \in \mathfrak{m}_{\widetilde{P}}(\mathrm{~F}) \cap \mathfrak{o}} \sum_{\gamma \in N_{P}^{Q}(\mathrm{~F})} \int_{\mathcal{V}_{\widetilde{P}}(\mathbb{A})} f_{\widetilde{Q}}\left(\operatorname{Ad}\left((\gamma \delta m)^{-1}\right)(\xi+Y)\right) d Y
$$

où $f_{\widetilde{Q}} \in \mathcal{S}\left(\mathfrak{m}_{\widetilde{Q}}(\mathbb{A})\right)$ est définie par (3.7) dans le paragraphe 3.2. D'autre part, l'intégrale sur $A_{\widetilde{Q}}^{s t, \infty}$ se réduit, grâce à l'identité (1.11) et le lemme 3.1, à

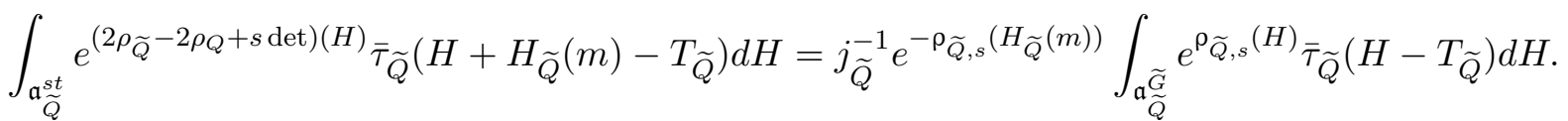

L'intégrale ci-dessus converge pour $s \in \mathbb{C}$ tels que $-1<\operatorname{Re}(s)<1$ en vertu du lemme 3.2 ii) et donne précisément $\hat{\theta}_{\widetilde{Q}}\left(\rho_{\widetilde{Q}, s}\right)^{-1} e^{\rho_{\widetilde{Q}, s}\left(T_{\widetilde{Q}}\right)}$ où $\hat{\theta}_{\widetilde{Q}}=\hat{\theta}_{\widetilde{Q}}^{\widetilde{G}}$ est définie par (3.3) dans le paragraphe 3.1 .

En utilisant alors l'égalité (5.15), on s'aperçoit que l'intégrale (5.16) égale $J_{\mathfrak{o}}^{M_{\widetilde{Q}}, T}\left(\eta_{s}, f_{\widetilde{Q}}\right)$ fois $j_{\widetilde{Q}}^{-1} \hat{\theta}_{\widetilde{Q}}\left(\rho_{\widetilde{Q}, s}\right)^{-1} e^{\rho_{\widetilde{Q}, s}\left(T_{\widetilde{Q}}\right)}$.

On a donc la convergence de l'intégrale dans le théorème ainsi que pour tout $T \in \mathfrak{a}_{\tilde{0}}^{+}$suffisamment régulier et tout $s \in \mathbb{C}$ tel que $-1<\operatorname{Re}(s)<1$

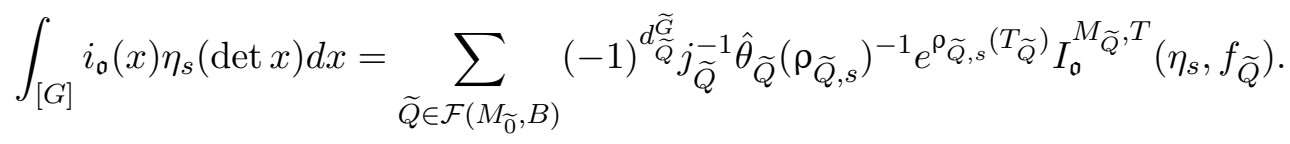

D'après le théorème 3.8 la somme ci-dessus égale $I_{\mathfrak{o}}\left(\eta_{s}, f\right)$, ce qu'il fallait démontrer.

\subsection{Résultats d'holomorphie}

Soient $\left\{e_{i}^{\vee}\right\}_{i \in I_{0} \cup-I_{0}} \subseteq \mathfrak{a}_{I_{0}}$ les vecteurs tels que

$$
\rho_{j}\left(e_{i}^{\vee}\right)=\left\{\begin{array}{l}
1 \text { si } j=i, \\
-1 \text { si } j=-i, \quad i, j \in I_{0} \cup-I_{0} . \\
0 \text { sinon, }
\end{array}\right.
$$

On a donc $e_{i}^{\vee}=-e_{-i}^{\vee}$ pour tout $i \in I_{0}$. En plus, il est clair que pour tout $\mathcal{I} \subseteq_{\epsilon} I_{0}$ l'ensemble $\left\{e_{i}^{\vee}\right\}_{i \in \mathcal{I}}$ est une base de $\mathfrak{a}_{|\mathcal{I}|}$.

Soit $\mathcal{I} \subseteq_{\epsilon} I_{0}$. On introduit le cône ouvert $\mathfrak{a}_{\mathcal{I}}^{*}$ défini comme

$$
\mathfrak{a}_{\mathcal{I}}^{*}=\left\{\sum_{i \in \mathcal{I}} a_{i} \rho_{i} \mid a_{i}>0\right\} \subseteq \mathfrak{a}_{|\mathcal{I}|}^{*} \subseteq \mathfrak{a}_{I_{0}}^{*}
$$

Donc, pour qu'un $\lambda \in \mathfrak{a}_{|\mathcal{I}|, \mathbb{C}}^{*}$ vérifie $\operatorname{Re}(\lambda) \in \mathfrak{a}_{\mathcal{I}}^{*}$ il faut et suffit que $\operatorname{Re}\left(\lambda\left(e_{i}^{\vee}\right)\right)>0$ pour tout $i \in \mathcal{I}$.

Soit $I^{\prime} \subseteq I$. Pour $\lambda \in \mathfrak{a}_{I, \mathbb{C}}^{*}$ on note $\lambda_{I^{\prime}}$ sa restriction à $\mathfrak{a}_{I^{\prime}, \mathbb{C}}^{*}$. Remarquons aussi que le caractère det est naturellement un élément de $\mathfrak{a}_{I^{\prime}, \mathbb{C}}^{*}$. Il vérifie det $=\sum_{i \in I^{\prime}} \rho_{i}$.

On rappelle que nous avons défini $I_{\eta}$ comme l'ensemble de $i \in I_{0}$ tels que la restriction de $\eta$ au groupe de normes de $\mathbb{A}_{i}^{*}$ dans $\mathbb{A}^{*}$ est non-trivial. Pour $I^{\prime} \subseteq I_{0}$ notons

$$
c_{I^{\prime}}=\int_{T_{I^{\prime}}(\mathrm{F}) \backslash T_{I^{\prime}}(\mathbb{A})^{1}} \eta(\operatorname{det} t) d t .
$$


On voit donc que si $I^{\prime} \cap I_{\eta} \neq \varnothing$ alors $c_{I^{\prime}}=0$. Notons aussi $v_{I^{\prime}}$ le volume dans $\mathfrak{a}_{I^{\prime}}$ du parallélotope déterminé par les vecteurs $\left\{e_{i}^{\vee}\right\}_{i \in \mathcal{I}}$ où $\mathcal{I} \subseteq_{\epsilon} I^{\prime}$ est tel que $|\mathcal{I}|=I^{\prime}$. Cela ne dépend pas du choix de $\mathcal{I}$. Notons aussi pour tout $i \in I^{\prime}$ :

$$
\mathcal{D}_{I^{\prime}, i, \mathbb{C}}^{*}:=\left\{\lambda \in \mathfrak{a}_{I^{\prime}, \mathbb{C}}^{*} \mid \lambda\left(e_{i}^{\vee}\right)=0\right\}=\left\{\lambda \in \mathfrak{a}_{I^{\prime}, \mathbb{C}}^{*} \mid \lambda\left(e_{-i}^{\vee}\right)=0\right\} .
$$

Lemme 5.18. Soit $\mathcal{I} \subseteq_{\epsilon} I_{0}$. Alors, pour tout $x \in G(\mathbb{A})$, l'intégrale

$$
\int_{T_{|\mathcal{I}|}(\mathrm{F}) \backslash T_{|\mathcal{I}|}(\mathbb{A})} \mathbb{1}_{\mathcal{I}}\left(H_{I}(t x)\right) e^{\lambda\left(H_{I}(t x)\right)} \eta(\operatorname{det} t) d t, \quad \lambda \in \mathfrak{a}_{|\mathcal{I}|, \mathbb{C}}^{*}
$$

converge absolument et uniformément sur tous les compacts de $\operatorname{Re}(\lambda) \in \mathfrak{a}_{\mathcal{I}}^{*}$. Elle admet un prolongement méromorphe, noté $\eta_{\mathcal{I}}$, égale à

$$
\eta_{\mathcal{I}}(\lambda)=(-1)^{\# \mathcal{I}} c_{|\mathcal{I}|} v_{|\mathcal{I}|} \prod_{i \in \mathcal{I}} \lambda\left(e_{i}^{\vee}\right)^{-1}
$$

En particulier, $\eta_{\mathcal{I}}$ ne dépend pas de $x \in G(\mathbb{A})$ et vaut 0 si $|\mathcal{I}| \cap I_{\eta} \neq \varnothing$. Si $|\mathcal{I}| \cap I_{\eta}=\varnothing$ l'ensemble $\left\{\mathcal{D}_{|\mathcal{I}|, i, \mathbb{C}}^{*}\right\}_{i \in|\mathcal{I}|}$ est l'ensemble de tous les hyperplans singuliers de $\eta_{\mathcal{I}}$.

Démonstration. Calcul direct.

Lemme 5.19. Soient $\mathcal{J}, \mathcal{J}_{1}, \mathcal{J}_{2}, \mathcal{J}_{3} \subseteq I_{0}$ tels que $\mathcal{J}_{1} \sqcup \mathcal{J}_{3} \subseteq \mathcal{J}$ et $\mathcal{J}_{2} \subseteq \mathcal{J}_{3}$.

i) L'intégrale suivante

$$
\Upsilon_{\mathcal{J}_{1}, \mathcal{J}_{2}, \mathcal{J}_{3}}(\eta, f)(\lambda)=\int_{T_{I_{0} \backslash\left|\mathcal{J}_{12}\right|}(\mathbb{A}) \backslash G(\mathbb{A})} \mathbb{1}_{\mathcal{J}_{1} \cup \mathcal{J}_{2}^{\sharp}}\left(H_{I}(x)\right) e^{\left(\lambda+\rho_{\mathcal{J}_{3 \backslash 2}^{\sharp}}\right)\left(H_{I}(x)\right)} \hat{f}_{x}^{\mathcal{J}_{3}}\left(X_{\mathcal{J}_{1} \cup \mathcal{J}_{2}^{\sharp}}\right) \eta(\operatorname{det} x) d x,
$$

où $\lambda \in \mathfrak{a}_{\left|\mathcal{J}_{12}\right|, \mathbb{C}}^{*}$, converge absolument et uniformément sur tous les compacts de $\operatorname{Re}(\lambda) \in \mathfrak{a}_{\left|\mathcal{J}_{12}\right|}^{*}$ et définit une fonction holomorphe sur $\mathfrak{a}_{\left|\mathcal{J}_{12}\right|, \mathbb{C}}^{*}$.

ii) L'intégrale suivante

$$
\left.\int_{T \mathcal{J}_{\mathcal{J}} \backslash \mathcal{J}_{12} \mid} \mathbb{1}_{(\mathcal{J}) T_{I_{0} \backslash|\mathcal{J}|}(\mathbb{A}) \backslash G(\mathbb{A})} \mathcal{J}_{2}\right) \cup \mathcal{J}_{2}^{\sharp}\left(H_{I}(x)\right) e^{\lambda\left(H_{I}(x)\right)} \hat{f}_{x}^{\mathcal{J}_{3}}\left(X_{\mathcal{J}_{1} \cup \mathcal{J}_{2}^{\sharp}}\right) \eta(\operatorname{det} x) d x, \quad \lambda \in \mathfrak{a}_{|\mathcal{J}|, \mathbb{C}}^{*}
$$

converge absolument et uniformément sur tous les compacts de

$$
\operatorname{Re}(\lambda) \in \mathfrak{a}_{\left|\mathcal{J}_{12}\right|}^{*} \times\left(\rho_{\mathcal{J}_{3 \backslash 2}^{\sharp}}+\mathfrak{a}_{\mathcal{J} \backslash \mathcal{J}_{12}}^{*}\right)
$$

et elle admet un prolongement méromorphe à $\mathfrak{a}_{|\mathcal{J}|, \mathbb{C}}^{*}$, noté $\bar{\Lambda}_{\mathcal{J}_{1}, \mathcal{J}_{2}, \mathcal{J}_{3}}^{\mathcal{J}}(\eta, f)$ qui vérifie

$$
\bar{\Lambda}_{\mathcal{J}_{1}, \mathcal{J}_{2}, \mathcal{J}_{3}}^{\mathcal{J}}(\eta, f)(\lambda)=\eta_{\mathcal{J} \backslash \mathcal{J}_{12}}\left(\lambda_{\left|\mathcal{J} \backslash \mathcal{J}_{12}\right|}+\rho_{\mathcal{J}_{3 \backslash 2}}\right) \Upsilon_{\mathcal{J}_{1}, \mathcal{J}_{2}, \mathcal{J}_{3}}(\eta, f)\left(\lambda_{\left|\mathcal{J}_{12}\right|}\right) .
$$

$S i\left|\mathcal{J} \backslash \mathcal{J}_{12}\right| \cap I_{\eta} \neq \varnothing$ on a $\bar{\Lambda}_{\mathcal{J}_{1}, \mathcal{J}_{2}, \mathcal{J}_{3}}^{\mathcal{J}}(\eta, f) \equiv 0$ et dans le cas contraire, l'ensemble :

$$
\left\{\mathcal{D}_{|\mathcal{J}|, i, \mathbb{C}}^{*}\right\}_{i \in\left|\mathcal{J} \backslash \mathcal{J}_{13}\right|} \cup\left\{\mathcal{D}_{|\mathcal{J}|,|i|, \mathbb{C}}^{*}+\rho_{-i}\right\}_{i \in \mathcal{J}_{3 \backslash 2}}
$$

où les hyperplans $\mathcal{D}_{|\mathcal{J}|, i, \mathbb{C}}^{*}$ sont définies par (5.18), est l'ensemble de tous les hyperplans singuliers de $\bar{\Lambda}_{\mathcal{J}_{1}, \mathcal{J}_{2}, \mathcal{J}_{3}}^{\mathcal{J}}(\eta, f)$. 
Démonstration. Le premier point c'est le lemme 5.22 de [Zyd15a]. Les assertions sur la convergence, le prolongement méromorphe ainsi que l'équation fonctionelle dans le deuxième point découlent des lemmes 5.24 et 5.25 de loc. cit. Finalement, la propriété d'annulation de $\bar{\Lambda}_{\mathcal{J}_{1}, \mathcal{J}_{2}, \mathcal{J}_{3}}^{\mathcal{J}}(\eta, f)$ et l'assertion sur ses éventuels hyperplans singuliers découlent de l'équation fonctionnelle qu'elle vérifie et de la propriété analogue de la fonction $\eta_{\mathcal{J} \backslash \mathcal{J}_{12}}$ décrite dans le lemme 5.18 ci-dessus.

Proposition 5.20. Soit $\mathcal{J} \subseteq_{\epsilon} I_{0}$. Alors, l'intégrale

$$
\int_{G\left(\mathbb{A}, X_{\mathcal{J}}\right) \backslash G(\mathbb{A})} f\left(x^{-1} X_{\mathcal{J}} x\right) e^{\lambda\left(H_{I}(x)\right)} \eta(\operatorname{det} x) d x, \quad \lambda \in \mathfrak{a}_{|\mathcal{J}|, \mathbb{C}}^{*}
$$

converge absolument et uniformément sur tous les compacts de $\operatorname{Re}(\lambda) \in \rho_{\mathcal{J}^{\sharp}}+\mathfrak{a}_{\mathcal{J}^{\sharp}}$ et admet un prolongement méromorphe à $\mathfrak{a}_{|\mathcal{J}|, \mathbb{C}}^{*}$, noté $\zeta_{\mathcal{J}}(\eta, f)$, qui vérifie

$$
\zeta_{\mathcal{J}}(\eta, f)=\sum_{\mathcal{J}_{1} \sqcup \mathcal{J}_{2} \sqcup \mathcal{J}_{3} \subseteq \mathcal{J}}(-1)^{\# \mathcal{J}_{3}} \bar{\Lambda}_{\mathcal{J}_{1}, \mathcal{J}_{2}, \mathcal{J}_{23}}^{\mathcal{J}}(\eta, f) .
$$

En plus, l'ensemble

$$
\left\{\mathcal{D}_{|\mathcal{J}|, i, \mathbb{C}}^{*}\right\}_{i \in|\mathcal{J}|} \cup\left\{\mathcal{D}_{|\mathcal{J}|,|i|, \mathbb{C}}^{*}+\rho_{-i}\right\}_{i \in \mathcal{J}},
$$

où les hyperplans $\mathcal{D}_{|\mathcal{J}|, i, \mathbb{C}}^{*}$ sont définies par (5.18), contient tous les hyperplans singuliers de $\zeta_{\mathcal{J}}(\eta, f)$

Démonstration. Convergence, prolongement méromorphe et l'équation fonctionnelle découlent de la proposition 5.26 accouplée avec le lemme 5.25 de Zyd15a. L'assertion sur les hyperplans singuliers est une conséquence de l'équation fonctionnelle vérifiée par $\zeta_{\mathcal{J}}(\eta, f)$ et du lemme 5.19 ci-dessus.

Dans le contexte du lemme 5.19 ci-dessus, posons $\bar{\Lambda}_{\mathcal{J}_{1}, \mathcal{J}_{2}}^{\mathcal{J}}(\eta, f)=\bar{\Lambda}_{\mathcal{J}_{1}, \mathcal{J}_{2}, \mathcal{J} \backslash \mathcal{J}_{1}}^{\mathcal{J}}(\eta, f)$.

Lemme 5.21. Soit $I^{\prime} \subseteq I$ et $\mathcal{J}_{0} \subseteq I_{\epsilon} I^{\prime} \cap I_{\eta}$ tel que $\left|\mathcal{J}_{0}\right|=I^{\prime} \cap I_{\eta}$. On a alors l'égalité de fonctions méromorphes sur $\mathfrak{a}_{I^{\prime}, \mathbb{C}}^{*}$ suivante

$$
\sum_{\left|\mathcal{I}_{0}\right|=I^{\prime} \backslash\left|I^{\prime} \cap I_{\eta}\right|} \zeta_{\mathcal{J}_{0} \cup \mathcal{I}_{0}}(f)(\eta, \lambda)=\sum_{\mathcal{J}_{0} \subseteq \mathcal{J}_{1} \sqcup \mathcal{J}_{2} \subseteq \subseteq^{\prime} I^{\prime}} \sum_{\left|\mathcal{J}_{3}\right|=I^{\prime} \backslash\left|\mathcal{J}_{12}\right|}(-1)^{\# \mathcal{J}_{3}} \bar{\Lambda}_{\mathcal{J}_{1}, \mathcal{J}_{2}}^{\mathcal{J}_{123}}(f)(\eta, \lambda) .
$$

Démonstration. Soient $\mathcal{J}_{1}, \mathcal{J}_{2}, \mathcal{J}_{3}, \mathcal{I}_{0} \subseteq I_{\epsilon} I^{\prime} \backslash\left|I^{\prime} \cap I_{\eta}\right|$ tels que $\left|\mathcal{I}_{0}\right|=I^{\prime} \backslash\left|I^{\prime} \cap I_{\eta}\right|$ et $\mathcal{J}_{1} \sqcup \mathcal{J}_{2} \sqcup \mathcal{J}_{3} \subseteq$ $\mathcal{I}_{0}$. En vertu du lemme 5 .19 ii) on a que si $\mathcal{J}_{0} \nsubseteq \mathcal{J}_{1} \sqcup \mathcal{J}_{2}$ alors $\bar{\Lambda}_{\mathcal{J}_{1}, \mathcal{J}_{2}, \mathcal{J}_{23}}(\eta, f) \equiv 0$. En vertu de la proposition 5.20 on a donc :

$$
\begin{aligned}
& \sum_{\left|\mathcal{I}_{0}\right|=I^{\prime} \backslash\left|I^{\prime} \cap I_{\eta}\right|} \zeta_{\mathcal{J}_{0} \cup \mathcal{I}_{0}}(f)(\eta, \lambda)=\sum_{\left|\mathcal{I}_{0}\right|=I^{\prime} \backslash\left|I^{\prime} \cap I_{\eta}\right|} \sum_{\mathcal{J}_{0} \subseteq \mathcal{J}_{1} \sqcup \mathcal{J}_{2} \subseteq \mathcal{J}_{0} \cup \mathcal{I}_{0}} \sum_{\mathcal{J}_{3} \subseteq\left(\mathcal{J}_{0} \cup \mathcal{I}_{0}\right) \backslash \mathcal{J}_{12}}(-1)^{\# \mathcal{J}_{3}} \bar{\Lambda}_{\mathcal{J}_{1}, \mathcal{J}_{2}, \mathcal{J}_{23}}^{\mathcal{J}_{\mathcal{I}^{\prime}} \mathcal{I}_{0}}(\eta, f)= \\
& \sum_{\mathcal{J}_{0} \subseteq \mathcal{J}_{1} \sqcup \mathcal{J}_{2} \subseteq_{\epsilon} I^{\prime}} \sum_{\mathcal{J}_{3} \subseteq I^{\prime} \backslash\left|\mathcal{J}_{12}\right|}(-1)^{\# \mathcal{J}_{3}} \sum_{\mathcal{J}_{4} \subseteq_{\epsilon} I^{\prime} \backslash\left|\mathcal{J}_{123}\right|} \bar{\Lambda}_{\mathcal{J}_{1}, \mathcal{J}_{2}, \mathcal{J}_{23}}^{\mathcal{J}_{123}}(\eta, f) .
\end{aligned}
$$

Il résulte de l'égalité (5.27) du lemme 5.27 de $\left[\right.$ Zyd15a que si $I^{\prime} \backslash\left|\mathcal{J}_{123}\right| \neq \varnothing$ on a

$$
\sum_{\mathcal{J}_{4} \subseteq \epsilon I^{\prime} \backslash\left|\mathcal{J}_{123}\right|} \bar{\Lambda}_{\mathcal{J}_{1}, \mathcal{J}_{2}, \mathcal{J}_{23}}^{\mathcal{J}_{123}}(\eta, f) \equiv 0
$$

D'où le résultat voulu.

Lemme 5.22. Pour tout $\mathcal{I} \subseteq \subseteq_{6} I_{0}$ non-vide et tout $i \in \mathcal{I}$ la droite $\mathbb{C}$ det n'est pas contenu dans $\mathcal{D}_{|\mathcal{J}|,|i|, \mathbb{C}}^{*} \cup\left(\mathcal{D}_{|\mathcal{J}|,|i|, \mathbb{C}}^{*}+\rho_{-i}\right)$. 
Démonstration. Il suffit de vérifier qu'il existe un $s \in \mathbb{C}$ tel que $s \operatorname{det}\left(e_{|i|}^{\vee}\right) \neq 0$ et $\left(s \operatorname{det}+\rho_{i}\right)\left(e_{|i|}^{\vee}\right) \neq$ 0 . Mais $s \operatorname{det}\left(e_{|i|}^{\vee}\right)=s$ et $\left(s \operatorname{det}+\rho_{i}\right)\left(e_{|i|}^{\vee}\right)=s+\frac{i}{|i|}$, d'où le résultat.

Soient $\mathcal{J}, \mathcal{J}_{1}, \mathcal{J}_{2} \subseteq_{\epsilon} I_{0}$ tels que $\mathcal{J}_{1} \sqcup \mathcal{J}_{2} \subseteq \mathcal{J}$. Posons :

$$
\bar{\Lambda}_{\mathcal{J}_{1}, \mathcal{J}_{2}}^{\mathcal{J}}(\eta, f)(s)=\bar{\Lambda}_{\mathcal{J}_{1}, \mathcal{J}_{2}}^{\mathcal{J}}(\eta, f)(s \operatorname{det}), \quad s \in \mathbb{C} .
$$

En vertu des lemmes 5.19 et 5.22, la fonction $\bar{\Lambda}_{\mathcal{J}_{1}, \mathcal{J}_{2}}^{\mathcal{J}}(\eta, f)(s)$ de la variable $s \in \mathbb{C}$ est méromorphe sur $\mathbb{C}$. On a alors le corollaire du lemme 5.19 suivant :

Corollaire 5.23. Soient $\mathcal{J}, \mathcal{J}_{1}, \mathcal{J}_{2} \subseteq I_{\epsilon}$ tels que $\mathcal{J}_{1} \sqcup \mathcal{J}_{2} \subseteq \mathcal{J}$.

i) Pour $-1<\operatorname{Re}(s)<1$, la fonction $\bar{\Lambda}_{\mathcal{J}_{1}, \mathcal{J}_{2}}^{\mathcal{J}}(\eta, f)(s)$ est donnée par l'intégrale absolument convergente suivante :

$$
\int_{T_{I_{0} \backslash \backslash \mathcal{J}_{12} \mid}(\mathrm{F}) \backslash G(\mathbb{A})} \mathbb{1}_{\left(\mathcal{J} \backslash \mathcal{J}_{2}\right) \cup \mathcal{J}_{2}^{\sharp}}\left(H_{I}(x)\right) \hat{f}_{x}^{\mathcal{J} \backslash \mathcal{J}_{1}}\left(X_{\mathcal{J}_{1} \cup \mathcal{J}_{2}^{\sharp}}\right) \eta_{s}(\operatorname{det} x) d x .
$$

ii) La fonction $\bar{\Lambda}_{\mathcal{J}_{1}, \mathcal{J}_{2}}^{\mathcal{J}}(\eta, f)(s)$ est holomorphe sur $s \in \mathbb{C} \backslash\{-1,1\}$.

iii) $S i\left|\mathcal{J} \backslash \mathcal{J}_{12}\right| \cap I_{\eta} \neq \varnothing$ on $a \bar{\Lambda}_{\mathcal{J}_{1}, \mathcal{J}_{2}}^{\mathcal{J}}(\eta, f)(s) \equiv 0$.

Démonstration. Pour démontrer le point $i$ ), en vertu du lemme 5.19 il suffit de vérifier

$$
\operatorname{Re}(s \text { det }) \in \mathfrak{a}_{\left|\mathcal{J}_{12}\right|}^{*} \times\left(\rho_{\left(\mathcal{J} \backslash \mathcal{J}_{12}\right)^{\sharp}}+\mathfrak{a}_{\mathcal{J} \backslash \mathcal{J}_{12}}^{*}\right) \quad-1<\operatorname{Re}(s)<1 .
$$

Autrement dit, on doit avoir $\operatorname{Re}(s) \operatorname{det}-\rho_{\left(\mathcal{J} \backslash \mathcal{J}_{12}\right)^{\sharp}} \in \mathfrak{a}_{\mathcal{J} \backslash \mathcal{J}_{12}}^{*}$. Or, on a que la restriction de det à $\mathfrak{a}_{\left|\mathcal{J} \backslash \mathcal{J}_{12}\right|}$ égale $\sum_{i \in \mathcal{J} \backslash \mathcal{J}_{12}} \rho_{|i|}=\sum_{i \in \mathcal{J} \backslash \mathcal{J}_{12}} \frac{i}{|i|} \rho_{i}$ et $\rho_{\left(\mathcal{J} \backslash \mathcal{J}_{12}\right)^{\sharp}}=\sum_{i \in \mathcal{J} \backslash \mathcal{J}_{12}}-\rho_{i}$. Par définition de $\mathfrak{a}_{\mathcal{J} \backslash \mathcal{J}_{12}}^{*}$ on a donc que (5.20) est vérifié si et seulement si pour tout $i \in \mathcal{J} \backslash \mathcal{J}_{12}$ on a $\operatorname{Re}(s) \frac{i}{|i|}>-1$. Comme $\frac{i}{|i|} \in\{-1,1\}$, l'assertion suit.

Pour le point ii), en utilisant l'équation fonctionnelle donnée dans le lemme 5.19, on a

$$
\bar{\Lambda}_{\mathcal{J}_{1}, \mathcal{J}_{2}}^{\mathcal{J}}(\eta, f)(s)=\eta_{\mathcal{J} \backslash \mathcal{J}_{12}}\left(s \operatorname{det}+\rho_{\mathcal{J} \backslash \mathcal{J}_{12}}\right) \Upsilon_{\mathcal{J}_{1}, \mathcal{J}_{2}, \mathcal{J}_{3}}(\eta, f)(s \operatorname{det})
$$

et la description explicite de $\eta_{\mathcal{J} \backslash \mathcal{J}_{12}}$ donnée dans le lemme 5.18 nous donne

$$
\eta_{\mathcal{J} \backslash \mathcal{J}_{12}}\left(s \operatorname{det}+\rho_{\mathcal{J} \backslash \mathcal{J}_{12}}\right)=(-1)^{\#\left(\mathcal{J} \backslash \mathcal{J}_{12}\right)} c_{\left|\mathcal{J} \backslash \mathcal{J}_{12}\right|} v_{\left|\mathcal{J} \backslash \mathcal{J}_{12}\right|} \prod_{j \in \mathcal{J} \backslash \mathcal{J}_{12}}\left(1+\frac{i}{|i|} s\right)^{-1} .
$$

Puisque $1+\frac{i}{|i|} s=0$ implique $s \in\{-1,1\}$ on a l'holomorphie de $\bar{\Lambda}_{\mathcal{J}_{1}, \mathcal{J}_{2}}^{\mathcal{J}}(\eta, f)(s)$ sur $\mathbb{C} \backslash\{-1,1\}$, vu l'holomorphie de $\mathbb{C} \ni s \mapsto \Upsilon_{\mathcal{J}_{1}, \mathcal{J}_{2}, \mathcal{J}_{3}}(\eta, f)$ (s det).

Le point iii) découle de l'équation fonctionnelle (5.21) ci-dessus.

Pour $\mathcal{J} \subseteq_{\epsilon} I$ on définit la fonction méromorphe $\zeta_{\mathcal{J}}(\eta, f)$ sur $\mathbb{C}$ par

$$
\zeta_{\mathcal{J}}(\eta, f)(s)=\zeta_{\mathcal{J}}(\eta, f)(s \text { det }), \quad s \in \mathbb{C} .
$$

En vertu de la proposition 5.20 et du lemme 5.22 la définition est licite.

On a alors le corollaire suivant du lemme 5.21 et du corollaire 5.23 .

Corollaire 5.24. Soit $I^{\prime} \subseteq I$ et $\mathcal{J}_{0} \subseteq_{\epsilon} I^{\prime} \cap I_{\eta}$ tel que $\left|\mathcal{J}_{0}\right|=I^{\prime} \cap I_{\eta}$. On a alors l'égalité de fonctions méromorphes sur $\mathbb{C}$ suivante

$$
\sum_{\left|\mathcal{I}_{0}\right|=I^{\prime} \backslash\left|I^{\prime} \cap I_{\eta}\right|} \zeta_{\mathcal{J}_{0} \cup \mathcal{I}_{0}}(\eta, f)=\sum_{\mathcal{J}_{0} \subseteq \mathcal{J}_{1} \sqcup \mathcal{J}_{2} \subseteq} \sum_{\mid I^{\prime}}(-1)^{\# \mathcal{J}_{3}\left|=I^{\prime} \backslash\right| \mathcal{J}_{12} \mid} \bar{\Lambda}_{\mathcal{J}_{1}, \mathcal{J}_{2}}^{\mathcal{J}_{123}}(\eta, f) .
$$

En particulier, en vertu du point ii) du corollaire 5.23, la somme ci-dessus est holomorphe sur $\mathbb{C} \backslash\{-1,1\}$. 


\section{Références}

[Art78] James Arthur. A trace formula for reductive groups. I. Terms associated to classes in $G(\mathbf{Q})$. Duke Mathematical Journal, 45(4) :911-952, 1978.

[Art81] James Arthur. The trace formula in invariant form. Ann. of Math. (2), 114(1) :1-74, 1981.

[Cha02] Pierre-Henri Chaudouard. La formule des traces pour les algèbres de Lie. Mathematische Annalen, 322(2) :347-382, 2002.

[GGP12] Wee Teck Gan, Benedict H. Gross, and Dipendra Prasad. Symplectic local root numbers, central critical $L$ values, and restriction problems in the representation theory of classical groups. Astérisque, (346) :1-109, 2012. Sur les conjectures de Gross et Prasad. I.

[God64] Roger Godement. Domaines fondamentaux des groupes arithmétiques. In Séminaire Bourbaki, 1962/63. Fasc. 3, No. 25\% 1964.

[Har14] R. Neal Harris. The refined Gross-Prasad conjecture for unitary groups. Int. Math. Res. Not. IMRN, (2) :303-389, 2014.

[II10] Atsushi Ichino and Tamutsu Ikeda. On the periods of automorphic forms on special orthogonal groups and the Gross-Prasad conjecture. Geom. Funct. Anal., 19(5) :1378-1425, 2010.

[IY] Atsuchi Ichino and Shunsuke Yamana. Periods of automorphic form : the case of $\left(\mathrm{Gl}_{\mathrm{n}+1} \times \mathrm{Gl}_{\mathrm{n}}, \mathrm{Gl}_{\mathrm{n}}\right)$. Compositio Math. À apparaître.

[JR11] Hervé Jacquet and Stephen Rallis. On the Gross-Prasad conjecture for unitary groups. In On certain L-functions, volume 13 of Clay Math. Proc., pages 205-265. Amer. Math. Soc., 2011.

[KMRT98] Max-Albert Knus, Alexander Merkurjev, Markus Rost, and Jean-Pierre Tignol. The book of involutions, volume 44 of American Mathematical Society Colloquium Publications. American Mathematical Society, Providence, RI, 1998. With a preface in French by J. Tits.

[LW09] Jean-Pierre Labesse and Jean-Loup Waldspurger. La formule des traces tordue d'après le Friday Morning Seminar. Paris, 2009.

[Mih15] Andreas Mihatsch. On the Arithmetic Fundamental Lemma through Lie algebras. Prépublication arXiv :1502.02855v1, 2015.

[RTZ13] Michael Rapoport, Ulrich Terstiege, and Wei Zhang. On the arithmetic fundamental lemma in the minuscule case. Compos. Math., 149(10):1631-1666, 2013.

[SG08] Rallis Steve and Schiffman Gérard. Multiplicity One Conjectures. Prépublication arXiv :0705.21268v1, 2008.

[Zha12] Wei Zhang. On arithmetic fundamental lemmas. Invent. Math., 188(1) :197-252, 2012.

[Zha14a] Wei Zhang. Automorphic period and the central value of Rankin-Selberg $L$-function. J. Amer. Math. Soc., 27 :541-612, 2014.

[Zha14b] Wei Zhang. Fourier transform and the global Gan-Gross-Prasad conjecture for unitary groups. Ann. of Math. (2), 180(3) :971-1049, 2014. 
[ZRS15] Wei Zhang, Michael Rapoport, and Brian Smithling. On the arithmetic transfer conjecture for exotic smooth formal moduli spaces. Prépublication arXiv :1503.06520v2, 2015.

[Zyd15a] Michał Zydor. La variante infinitésimale de la formule des traces de Jacquet-Rallis pour les groupes unitaires. Prépublication arXiv :1306.1061v2, 2015.

[Zyd15b] Michał Zydor. Les formules des traces relatives de Jacquet-Rallis grossières. 2015. En préparation. 